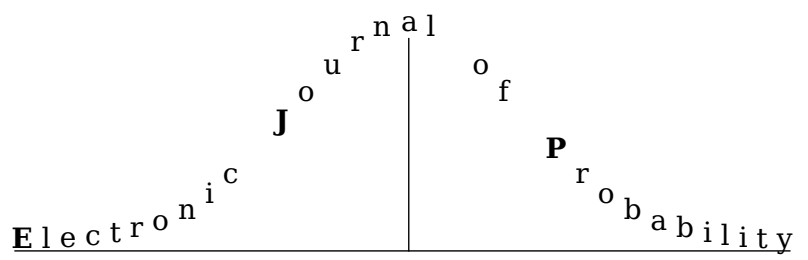

Electron. J. Probab. 27 (2022), article no. 27, 1-43.

ISSN: 1083-6489 https://doi.org/10.1214/22-EJP755

\title{
Spin distributions for generic spherical spin glasses
}

\author{
Arka Adhikari*
}

\begin{abstract}
This paper investigates spin distributions for a generic spherical $p$-spin model; we give a representation of spin distributions in terms of a stochastic process. In order to do this, we find a series of invariance principles analogous to the cavity equations used for the hypercube. The construction of these equations is a multistep process; we first change coordinates to write the sphere as a product space $S_{N-R^{2}} \times[-C, C]$ for some cutoff parameters $R$ and $C$. We then construct an auxiliary Hamiltonian that writes the effect of the final spin as an independent Gaussian process, e.g., $H_{N} \approx H_{N-1}+Y_{N}+Z_{N}$ with $Y_{N}$ and $Z_{N}$ decoupled from $H_{N-1}$. The error rate in this approximation decays as $O\left(\frac{C^{4}}{R^{2}}\right)$. The final step is to justify the double limit $\lim _{C \rightarrow \infty} \lim _{R \rightarrow \infty}$ to derive exact results; this double limit can be interpreted as a novel renormalization procedure.
\end{abstract}

Keywords: spin glasses; spin distributions; invariance principle.

MSC2020 subject classifications: 82B20.

Submitted to EJP on April 26, 2021, final version accepted on February 9, 2022.

Supersedes arXiv: 1907.03847.

\section{Introduction}

Spin glasses are used as models for understanding the properties of disordered magnets, where, unlike ferromagnetic and paramagnetic models, the directions of the magnetic fields are oriented arbitrarily. In 1975, Sherrington and Kirkpatrick proposed a mean field model in order to gain a foothold on some of the more computational aspects of the problem. The SK Hamiltonian [15] is

$$
H_{N}(\sigma)=\frac{1}{\sqrt{N}} \sum_{1 \leq i<j \leq N}^{N} J_{i j} \sigma_{i} \sigma_{j},
$$

where $\sigma=\left(\sigma_{1}, \ldots, \sigma_{N}\right) \in\{-1,1\}^{N}$, and $J_{i j}$ are i.i.d. $N(0,1)$.

\footnotetext{
${ }^{*}$ Harvard University, United States of America. E-mail: adhikari@math . harvard.edu
} 
Through this Hamiltonian, we can define a probability measure on the hypercube; for a given configuration of the random couplings $J_{i j}$, one can define the Gibbs measure, $\mathcal{G}_{N}$, which is given via the following formula.

$$
\mathcal{G}_{N}(\sigma)=\frac{e^{\beta H_{N}(\sigma)}}{Z_{N}},
$$

where the partition function $Z_{N}$ is a normalization factor

$$
Z_{N}:=\sum_{\sigma \in\{-1,1\}^{N}} e^{\beta H_{N}(\sigma)} .
$$

The study of the Sherrington-Kirkpatrick model revealed multiple physically novel aspects of spin glasses that are not present in other magnetic models. Even the limiting free energy, defined as $f:=\lim _{N \rightarrow \infty} \frac{1}{N} \log Z_{N}$ is a highly non-trivial computation at low temperatures. The limiting formula for the free energy is given by the Parisi formula [13,14], which can be represented as a minimization question over the set of all probability measures on $[0,1]$. To each probability measure $\mu$ in the set $\mathcal{M}$ of all probability measures supported on $[0,1]$, we can associate the following PDE:

$$
\begin{aligned}
& \partial_{t} \Psi_{\mu}(t, x)=-\frac{\beta^{2}}{2}\left(\partial_{x x} \Psi_{\mu}(t, x)+\mu([0, s])\left(\partial_{x} \Psi_{\mu}(t, x)\right)^{2}\right), \\
& \Psi_{\mu}(1, x)=\log \cosh x .
\end{aligned}
$$

The limiting free energy is then given by the expression:

$$
f=\inf _{\mu \in \mathcal{M}}\left(\log 2+\Psi_{\mu}(0,0)-\frac{1}{2} \int_{0}^{1} \beta^{2} s \mu([0, s]) \mathrm{d} s\right) .
$$

The variational parameter introduced in the Parisi formula is supposed to represent the limiting overlap distribution, $\zeta$, which is given by the following formula.

$$
\zeta(\cdot \in[a, b]):=\lim _{N \rightarrow \infty} \mathbb{E}\left[\mathcal{G}_{N}\left(\left\langle\sigma^{1}, \sigma^{2}\right\rangle \in[a, b]\right)\right] .
$$

Here, $\langle\cdot, \cdot\rangle$ is the standard inner product in $\mathbb{R}^{N}$ normalized by $\frac{1}{N}$ and $\sigma^{1}, \sigma^{2}$ are spin configurations chosen independently with respect to the Gibbs measure $\mathcal{G}_{N}$. Observe that in the above expression, we take the expectation over the randomness of the Gibbs measures. In general, the overlap is highly non-trivial for spin glasses. This is in contrast to either a ferromagnetic or paramagnetic model, where all the spins are either oriented in the same direction or oriented randomly. In these models with a more regular magnetization structure, one would expect the overlap distribution to concentrate either at 1 or at 0 .

An interpretation of the Parisi formula by Mezard, et al. [7-9], led to a complex picture for the geometry of the Gibbs measure. Under this interpretation, the Gibbs measure is organized in a hierarchical way like a tree. The tree starts to branch when the overlap structure changes; one can determine the overlap of two 'leaves' of the tree by the value associated with their most recent common ancestor. The Gibbs measure satisfies ultrametricity, which means that the following relation holds:

$$
\lim _{N \rightarrow \infty} \mathbb{E}\left[\mathcal{G}_{N}\left(\left\langle\sigma^{1}, \sigma^{2}\right\rangle \geq \min \left(\left\langle\sigma^{1}, \sigma^{3}\right\rangle,\left\langle\sigma^{2}, \sigma^{3}\right\rangle\right)\right)\right]=1 .
$$

The Parisi formula was proved by Talagrand in [20]; ultrametricity is not yet established for the $S K$ model itself, though Panchenko showed that generic models, those whose Hamiltonian includes all higher order spins, satisfy ultrametricity. 
Though the study of the free energy gave many insights into the physical nature of the Gibbs measure, the free energy to highest order by itself is not able to give very fine details about the Gibbs distribution. In this paper, we would like to consider the question of spin distributions; this means we will look at the behavior of individual coordinates $\sigma_{i}^{l}$ of our spin glass on the sphere.

Such information may be gleaned if one has access to higher order corrections to the free energy formula. In general, this is a difficult problem and requires a very precise analysis of explicit formulas as in $[17,18]$. For the question of spin distributions on the hypercube, Panchenko found a series of invariance principles called the cavity equations [10] that allows one to compute individual spin distributions under the assumption of finite replica symmetry breaking. Auffinger and Jagannath [4] used the cavity equations for the hypercube to derive the invariance principle. They then took a limit procedure to study the spin distributions of models that satisfy full replica symmetry breaking. They found a representation of the $p$-spin distributions on the hypercube in terms of a stochastic process defined on the support of the limiting Gibbs measure.

\subsection{Setting and related notation}

In this paper, we investigate the asymptotic spin distributions of a generic Hamiltonian on the sphere $S_{N}:=\left\{\left(x_{1}, \ldots, x_{N}\right): \sum_{i=1}^{N} x_{i}^{2}=N\right\}$. We will use the more general notation $S_{k}(r)$ to denote the $k$-dimensional sphere of radius $\sqrt{r}$. Writing the Hamiltonian in terms of its $p$-spin components, we have

$$
H_{N}(\sigma)=\sum_{p=2}^{\infty} \beta_{p} \frac{1}{N^{(p-1) / 2}} \sum_{i_{1}, \ldots, i_{p}} g_{i_{1}, \ldots, i_{p}} \sigma_{i_{1}} \sigma_{i_{2}} \ldots \sigma_{i_{p}} .
$$

We can alternatively define the Hamiltonian in terms of its covariance structure. For two points $\sigma=\left(\sigma_{1}, \sigma_{2}, \ldots, \sigma_{N}\right)$ and $\rho=\left(\rho_{1}, \rho_{2}, \ldots, \rho_{N}\right)$ we have the following Hamiltonian $H_{N}$

$$
\operatorname{Cov}\left(H_{N}(\sigma), H_{N}(\rho)\right)=N \xi\left(R_{12}\right),
$$

where the overlap $R_{12}$ between $\sigma$ and $\rho$ is given by

$$
R_{12}=\frac{1}{N} \sum_{i=1}^{N} \sigma_{i} \rho_{i}
$$

and $\xi$ is given by

$$
\xi(x)=\sum_{p=2}^{\infty} \beta_{p}^{2} x^{p} .
$$

We remark here that the right hand side is the normalized inner product of $\left(\sigma_{1}, \ldots, \sigma_{N}\right)$ and $\left(\rho_{1}, \ldots, \rho_{N}\right)$ as vectors in $\mathbb{R}^{N}$. We may interchangeably use the notation $\langle\sigma, \rho\rangle$ to represent this normalized inner product.

We have the following assumptions on the Hamiltonian $H_{N}$.

Assumption 1.1. - We assume the Hamiltonian $H_{N}$ is a generic model. This means that the span of the polynomials $x^{p}$ corresponding to those terms in the Hamiltonian (1.9) whose coefficients $\beta_{p} \neq 0$ would be dense in $C\left([-1,1],\|\cdot\|_{\infty}\right)$, the space of all continuous functions on $[-1,1]$ under the $L^{\infty}$ norm. We usually take $\beta_{p} \geq 0$. The importance of this assumption is that it allows us to apply Panchenko's Ultrametricity Theorem [12][Col 3.2,Thm 2.14]and establish the uniqueness of the overlap distribution.

-We have sufficient decay on the coefficients $\beta_{p}$; namely, we will assume that there exists some $\epsilon>0$ and some constant $K$ such that $\beta_{p} \leq \frac{K}{p^{\frac{3}{2}+\epsilon}}$. This is not necessarily 
the most general constraint, but this will allow one to perform perturbation theory more carefully.

For the spherical model, we no longer have the cavity equation. In this paper, we invent a new series of invariance principles and an appropriate limiting procedure using a renormalization argument to get a representation of the spin distributions. The representation produced here is parallel to the method of Lagrange multipliers used in papers like [19] and, notably, captures information about the joint distribution of the spins, such as tail probabilities, that are not clearly determined by the Lagrange multiplier representation.

\subsection{Notions from the theory of spin distributions}

Our main result will involve associating the limiting spin distributions $\sigma_{i}^{l}$ to an associated PDE process. To begin to describe this process, we need to start by describing some basic notation from the theory of spin distributions, as in [12][Chapter4].

For a fixed choice of the random couplings $g_{i_{1}, i_{2}, \ldots, i_{p}}$, let $\mathcal{G}_{N}$ be the Gibbs measure on $S_{N}$ corresponding to the spherical Hamiltonian $H_{N}$. We let $\left(\sigma^{l}\right), l \in \mathbb{Z}^{+}$be a series of replicas i.i.d. distributed according to $\mathcal{G}_{N}$. From this, we can construct both the spin matrix $\left(s^{N}\right)$ from the individual coordinates of the spins $\sigma^{l}$ as well as the overlap matrix $R^{N}$.

The first few coordinates of the matrix are given by the entries $\left(s^{N}\right)_{l, i}=\sigma_{i}^{l}, l \in$ $\mathbb{Z}^{+}, 1 \leq i \leq N$. It can be extended to an $\infty$ by $\infty$ matrix by setting $\left(s^{N}\right)_{l, i}=0$ for $i>N$. The overlap matrix is given by the $L^{2}$ inner products of the replicas as vectors in $\mathbb{R}^{N}$; $\left(R^{N}\right)_{l, l^{\prime}}=\left\langle\sigma^{l}, \sigma^{l^{\prime}}\right\rangle$. We let $\mu_{N}$ be the law of $s^{N}$ and $\eta_{N}$ be the law of $R^{N}$ when distributed according to $\mathbb{E}\left[\mathcal{G}_{N}^{\otimes \infty}\right]$. We will say that $\mu$ (respectively $\eta$ ) is a limiting spin (respectively overlap) distribution if it is a subsequential limit of some $\mu_{N}$ (respectively $\eta_{N}$ ) in the sense of the convergence of finite dimensional distributions.

Notice that $\eta$ is a weakly exchangeable distribution of positive semidefinite matrices. We can use the Dobvysh-Sudakov theorem [6] in order to say there is a random measure $\nu$ on $L^{2}[0,1] \times \mathbb{R}$ satisfying the following property. The distribution of the overlap matrix $\left(R_{l, l^{\prime}}\right)$ from $\eta$ is the same as the distribution of $\left(a_{l} \delta_{l, l^{\prime}}+\left\langle h_{l}, h_{l}^{\prime}\right\rangle\right)$ where $\left(h_{l}, a_{l}\right)$ are sampled i.i.d from $\nu$ conditionally on the realization of the random measure $\nu$. The measure $\nu$ restricted to the space $L^{2}[0,1]$ is called an asymptotic Gibbs measure.

For generic $p$-spin models, we can say more about the distribution $\eta$. Because of the Ghirlanda-Guerra Identities, it is known that the law of the distribution of the entire overlap matrix is a function of the law of the overlap distribution $R_{1,2}$ [12][Thm 2.13]. In addition, for generic $p$-spin models, the limiting distribution of $R_{1,2}$ is unique.

\subsection{The SDE representation}

It turns out that the asymptotic measures $\mu$ and $\nu$ can be expressed via a process associated with the solution of a corresponding Parisi partial differential equation. The limiting 'local magnetic field' of a spin glass is given by a stochastic process driven by a Brownian motion whose drift and diffusion coefficients depend on the Parisi PDE. Knowing the 'local field' will allow one to determine the spin distribution via a cavity-type computation. To determine the joint distribution of many spins, one computes the 'local field' for multiple particles driven by a Brownian field with an appropriate covariance structure. Proving the existence of this process is not difficult; it was shown for the hypercube in [4] and we can adapt the construction for the sphere.

Consider $q_{*}>0$ and let $U$ be a positive ultrametric subset of the sphere of radius $\sqrt{q_{*}}$ in the space $L^{2}[0,1]$. An ultrametric set $\left\{u_{1}, \ldots, u_{n}, \ldots\right\}$, is a subset that satisfies the inner product relations $\left\langle u_{i}, u_{j}\right\rangle \geq \min \left(\left\langle u_{i}, u_{k}\right\rangle,\left\langle u_{k}, u_{j}\right\rangle\right)$ for all triples $i, j, k$. We define a 
Brownian motion on $U$; consider the Gaussian process $B_{t}(\sigma)$ indexed by $(t, \sigma) \in\left[0, q_{*}\right] \times U$ which is centered, a.s. continuous in time and in space with covariance

$$
\operatorname{Cov}\left(B_{t_{1}}\left(\sigma_{1}\right), B_{t_{2}}\left(\sigma_{2}\right)\right)=\left(t_{1} \wedge t_{2}\right) \wedge\left(\sigma_{1}, \sigma_{2}\right),
$$

where $\left\langle\sigma_{1}, \sigma_{2}\right\rangle$ is the inner product between two states in $L^{2}[0,1]$.

Using this Brownian motion, we can define the cavity field $\mathcal{Z}_{t}(\sigma)$ on $U$ as the solution of the following SDE

$$
\begin{aligned}
& \mathrm{d} \mathcal{Z}_{t}(\sigma)=\sqrt{\xi^{\prime \prime}(t)} \mathrm{d} B_{t}(\sigma), \\
& \mathcal{Z}_{0}(\sigma)=0 .
\end{aligned}
$$

Let $\zeta$ be the Parisi measure for the generic spherical $p$-spin model. Consider the following Parisi initial value problem on $(0,1) \times \mathbb{R}$

$$
\begin{aligned}
& u_{t}^{\zeta}+\frac{\xi^{\prime \prime}(t)}{2}\left(u_{x x}^{\zeta}+\zeta([0, t])\left(u_{x}^{\zeta}\right)^{2}\right)=0, \\
& u^{\zeta}(1, x)=\frac{x^{2}}{2\left(1+b_{\zeta}\right)},
\end{aligned}
$$

where $b_{\zeta}$ is defined in terms of $\xi$ and $\zeta$ as

$$
b_{\zeta}=\int_{0}^{1} \zeta([0, l])\left(l \xi^{\prime \prime}(l)+\xi^{\prime}(l)\right) \mathrm{d} l .
$$

We can now define the local field $\mathcal{X}_{t}(\sigma)$ on $\left[0, q_{*}\right] \times U$ by an SDE

$$
\begin{aligned}
& \mathrm{d} \mathcal{X}_{t}(\sigma)=\xi^{\prime \prime}(t) \zeta([0, t]) u_{x}^{\zeta}\left(t, \mathcal{X}_{t}(\sigma)\right) \mathrm{d} t+\mathrm{d} \mathcal{Z}_{t}(\sigma), \\
& \mathcal{X}_{0}(\sigma)=0 .
\end{aligned}
$$

Many basic properties of these stochastic processes are covered in section A.1 of [4]. The Parisi formula for the sphere was proved in Theorem 1.1 of [19]. We have also constructed solutions to this Parisi initial value problem in section A.2 of the Appendix in this paper. We will explain how these concepts are used on an intuitive level, without trying to be too rigorous.

If we describe the organization of a spin glass as a tree according to the RPC structure, then $u^{\zeta}(t, x)$ represents the free energy of the subtree containing a node at height $t$ and all of its children under an external magnetic field of value $x$. For example, on the hypercube, $u(1, x)=\log \cosh x$, which is exactly the energy of a single particle at external field value $x$. If we knew the distribution of the magnetic field once it reached the final level $u(1,$.$) , then we could relate the free energy u(0, x)$ to the expected value $\mathbb{E}_{X}[u(1, X)]$ for some proposed magnetic field distribution $X$ at time 1 . The proposed distribution for $X$ is exactly the solution $\mathcal{X}_{1}$ to the equation (1.15); this is why we call $\mathcal{X}$ the 'local field'. For more details on this representation, one can refer to Theorem 3 of [3].

On the sphere, we can describe the spin as being distributed according to a Gaussian with variance given by some constant $\frac{1}{1+b}$ and exponentially tilted given by the magnetic field. Informally speaking, if we let $P(s)$ denote the probability distribution of a single spin, then we expect

$$
P(s) \propto e^{-\frac{1+b}{2} s^{2}} e^{s \mathcal{X}_{1}} .
$$

Apart from justifying the above representation for a single spin distribution, the problem of determining a spin distribution additionally involves determining the correlation structure of the 'local field' $\mathcal{X}_{1}$ between different replicas as well as the constant $b$ used in rescaling the variance of a single spin. The ultrametric subset $U$ represents the 
different replicas that we may consider; our spatial Brownian motion defined in (1.12) captures the correlation in $\mathcal{X}_{1}$ between these different replicas. The constant $b$ is usually phrased in terms of an optimization problem; the fact that we can derive the value directly from properties of the Hamiltonian and the overlap distribution is a significant novelty of the argument and illustrates the power of the renormalization approach taken.

We now return to the discussion of the main result. Using these stochastic processes that we have defined previously, we are now able to explicitly write out the spin distributions that we are considering. We define the measure $\rho_{\zeta, \sigma}^{f}$ where $\sigma$ is a state in $U$, our ultrametric subset, and $f$ is a fixed measurable function on $L^{2}[0,1]$, as follows. Let $g$ be a bounded function on $\mathbb{R}$ with compact support. Then $\rho_{\zeta, \sigma}^{f}$ is given by

$$
\rho_{\zeta, \sigma}^{f}(g)=\frac{\int_{-\infty}^{\infty} \int_{-\infty}^{\infty} g(s) e^{-\frac{\left[1+b_{\zeta}\right]}{2} s^{2}} e^{-\frac{(y-f(\sigma))^{2}}{2\left[\xi^{\prime}(1)-\xi^{\prime}\left(q_{*}\right)\right]}} e^{s y} \mathrm{~d} y \mathrm{~d} s}{\int_{-\infty}^{\infty} \int_{-\infty}^{\infty} e^{-\frac{\left[1+b_{\zeta}\right]}{2} s^{2}} e^{-\frac{(y-f(\sigma))^{2}}{2\left[\xi^{\prime}(1)-\xi^{\prime}\left(q_{*}\right)\right]}} e^{s y} \mathrm{~d} y \mathrm{~d} s}
$$

This differs slightly from the notation in [4] to denote the point where we are evaluating $f$. When we consider spin glasses whose overlap distributions $\zeta$ have support restricted to the interval $\left[0, q_{*}\right]$ with $q_{*}<1$, we see that we will consider magnetizations $\mathcal{X}_{q *}$ evaluated at time $q_{*}$ instead of 1 . On a heuristic level, the purpose of $\rho_{\zeta, \sigma}^{f}$ is to determine the spin distribution at time 1 based on information of the magnetization at time $q_{*}$. Informally speaking, $\mathcal{X}_{1}-\mathcal{X}_{q_{*}}$ is a Gaussian of variance $\xi^{\prime}(1)-\xi^{\prime}\left(q_{*}\right)$.

From this point on, we will use $\langle\cdot\rangle_{H_{N}}$ (with only a single element) to denote Gibbs' expectations with respect to some Hamiltonian and some set(in the example above, the Hamiltonian is $H_{N}$ and, when there is no further specification, the set is $S_{N}$ ). This is in contrast to the two element inner product $\langle\cdot, \cdot\rangle$. From this point on Gibbs expectations will appear far more frequently. We will establish the following theorem.

Theorem 1.2. Consider a spherical Hamiltonian $H_{N}$ satisfying the assumptions of Assumption 1.1. Let $\zeta^{*}(\cdot)=\lim _{N \rightarrow \infty} \mathbb{E}\left\langle I\left(R_{1,2} \in \cdot\right)\right\rangle_{H_{N}}$ be the limiting overlap distribution and let $q_{*}=\sup \operatorname{supp}\left(\zeta^{*}\right)$. Let $\mu$ be an asymptotic spin distribution and $\nu$ be an asymptotic Gibbs measure corresponding to $H_{N}$. Since $H_{N}$ is generic, $\nu$ is unique.

We define the following measure on $\infty \times \infty$ matrices: choose $\left\{\sigma^{l}\right\}$ i.i.d from $\nu^{\otimes \infty}$. Independently construct $\mathcal{X}_{t}^{i}(\sigma)$ distributed according to (1.15) on $U=\operatorname{supp}(\nu)$. Distribute $s_{i}^{l}$ according to the measure $\rho_{\zeta^{*}, \sigma^{l}}^{\mathcal{X}_{q^{l}}^{i}}$.

Let $\left(\tilde{s}_{i}^{l}\right)$ be spins distributed according to $\mu$. Then $\left(s_{l}^{i}\right)$ and $\left(\tilde{s}_{l}^{i}\right)$ are equal in distribution. As a consequence, this shows $\mu$ is also unique.

Remark 1.3. Here, we discuss the relationship between the constants $b_{\zeta}$ given here with some other relevant constants in the literature, namely the constant $b$ that Talagrand uses in [19] as a parameter to describe the free energy as in his equation (3.35). It was observed that this parameter $b$ determines the effective variance of the spins if they were to be treated as Gaussian variables. There are a number of relations used in Section 4 of the same paper that relate this constant $b$ to our constant $b_{\zeta}$.

In equation (4.12) of Lemma 4.1, he derives the following relationship between the quantities $A(q, b)$ and $B(q)$. These quantities become equal at the Parisi minimizer. He derives, for a model with finite replica symmetry breaking, the following relation at the Parisi minimizer

$$
0=A(q, b)-B(q)=b-1-\sum_{1 \leq l \leq k} m_{l}\left(q_{l+1} \xi^{\prime}\left(q_{l+1}\right)-q_{l} \xi^{\prime}\left(q_{l}\right)\right)+\Delta_{1} \xi^{\prime}\left(q_{1}\right)-\frac{q_{1}}{\Delta_{1}} .
$$

Later in the work, he derives for a model with no magnetization, which we are dealing 
with here, the relation (4.14), which we reproduce here as

$$
0=\frac{\mathrm{d} B(q)}{\mathrm{d} q_{1}}=-m_{1} \xi^{\prime}\left(q_{1}\right)-m_{1} h^{2}+\frac{m_{1} q_{1}}{\Delta_{1}^{2}}+\frac{1}{\Delta_{1}}-\frac{1}{\Delta_{1}} .
$$

Simplifying this at magnetization $h=0$ gives the relation $\Delta_{1} \xi^{\prime}\left(q_{1}\right)-\frac{q_{1}}{\Delta_{1}}=0$. Substituting this into the earlier expression gives

$$
b=1+\sum_{1 \leq l \leq k} m_{l}\left(q_{l+1} \xi^{\prime}\left(q_{l+1}\right)-q_{l} \xi^{\prime}\left(q_{l}\right)\right) .
$$

This is a discrete version of our constant $b_{\zeta}+1$ for models satisfying finite replica symmetry breaking.

\subsection{Overview of the strategy}

A set of invariance principles called the cavity equations [10] gives a way to understand the behavior of some individual spins. In the paper [4], the cavity equations resulting from the product structure of the hypercube was used to derive these invariance principles. To understand the sphere, a critical step is to establish an analog of these cavity equations.

The key point here is that, in order to understand the distribution of a single spin $\sigma_{N}$, one can separate the Hamiltonian $H_{N}$ into two parts

$$
H_{N}\left(\sigma_{1}, \ldots, \sigma_{N}\right)=H_{N-1}\left(\sigma_{1}, \ldots, \sigma_{N-1}\right)+h_{N}\left(\sigma_{1}, \ldots, \sigma_{N-1}, \sigma_{N}\right),
$$

where both $H_{N-1}$ and $h_{N}$ are Gaussian processes. $h_{N}$ consists of all terms in the Hamiltonian that involve the extra spin $\sigma_{N}$. We remark that on the hypercube, the process $h_{N}\left(\sigma_{1}, \ldots, \sigma_{N}\right)$ can be represented as $h_{N}\left(\sigma_{1}, \ldots, \sigma_{N}\right)=z_{N}\left(\sigma_{1}, \ldots, \sigma_{N-1}\right) \sigma_{N}$. Here, $z_{N}\left(\sigma_{1}, \ldots, \sigma_{N-1}\right)$ acts like a magnetic field on the final spin.

The success of the cavity method depends on two properties.

Definition 1.4. [Properties for the Cavity Equation]

1. The covariance structure of the Hamiltonian $H_{N-1}$

$$
\operatorname{Cov}\left(H_{N-1}\left(\sigma_{1}, \ldots, \sigma_{N-1}\right), H_{N-1}\left(\rho_{1}, \ldots, \rho_{N-1}\right)\right),
$$

does not depend on the value of $\sigma_{N}$ or $\rho_{N}$.

2. As Gaussian processes, $H_{N-1}$ and $h_{N}$ are independent.

Computations in the cavity method depend on modified Bolthausen-Sznitman invariance principles, [12][Section 4.4, Pg 131]. The specific definition of spin glasses on the hypercube allows a decomposition where both of these properties hold simultaneously.

On the sphere, one can immediately see that the value of $\sigma_{N}$ will, in turn, affect the value of $\sigma_{1}^{2}+\ldots+\sigma_{N-1}^{2}$. A naive decomposition, like what is done for the hypercube, would not satisfy item one of Definition 1.4. To deal with item one of Definition 1.4, we will first define a change of coordinates so that it would 'look' like a product space. We illustrate the process when one particle is removed.

We first choose a renormalization scale $R$ and a new reference sphere for the first $N-1$ coordinates. We also introduce a cutoff $C$ for the last spin, $\sigma_{N}$. Namely, we consider

$$
\hat{\sigma}_{1}^{2}+\ldots+\hat{\sigma}_{N-1}^{2}=N-R^{2}, \quad \text { with } \hat{\sigma}_{i}=\sqrt{\frac{N-R^{2}}{N-\sigma_{N}^{2}}} \sigma_{i}, \text { and }\left|\sigma_{N}\right| \leq C .
$$


We can now consider the original Hamiltonian on the $N$ dimensional sphere as a Hamiltonian of the product of an $N-1$ dimensional sphere and the interval $[-C, C]$ for the last coordinate $\sigma_{N}$. Before we proceed, it is important to remark that the scale $R$ is a free parameter. The introduction of the cutoff $C$ is important to regularize estimates; it has to be taken to $\infty$ in some limit to get the exact spin distribution.

These coordinates allows us to decompose the Hamiltonian up to leading order as

$$
\begin{aligned}
H_{N}\left(\sigma_{1}, \ldots, \sigma_{N}\right) & =\hat{H}_{N-1}\left(\hat{\sigma}_{1}, \ldots, \hat{\sigma}_{N-1}\right)+h_{N}\left(\hat{\sigma}_{1}, \ldots, \hat{\sigma}_{N-1}, \sigma_{N}\right), \\
\hat{H}_{N-1}\left(\hat{\sigma}_{1}, \ldots, \hat{\sigma}_{N-1}\right) & =\sum_{p=2}^{\infty} \frac{\beta_{p}}{N^{(p-1) / 2}} \sum_{1 \leq i_{1}, \ldots, i_{p} \leq N-1} g_{i_{1}, \ldots, i_{p}} \hat{\sigma}_{i_{1}} \ldots \hat{\sigma}_{i_{p}}, \\
h_{N}\left(\hat{\sigma}_{1}, \ldots, \hat{\sigma}_{N-1}, \sigma_{N}\right) & =\sum_{p=2}^{\infty} \frac{\beta_{p}}{N^{(p-1) / 2}} \sum_{1 \leq i_{1}, \ldots, i_{p} \leq N-1}\left(\mathcal{S}^{p}-1\right) g_{i_{1}, \ldots, i_{p}} \hat{\sigma}_{i_{1}} \ldots \hat{\sigma}_{i_{p}} \\
& +\sum_{p=2}^{\infty} \frac{\beta_{p}}{N^{(p-1) / 2}} \sum_{1 \leq i_{1}, \ldots, i_{p-1} \leq N-1} g_{i_{1}, \ldots, i_{p-1}, N} \hat{\sigma}_{i_{1}} \ldots \hat{\sigma}_{i_{p-1}} \sigma_{N} .
\end{aligned}
$$

Here, $\mathcal{S}$ is the factor $\sqrt{\frac{N-\sigma_{N}^{2}}{N-R^{2}}}$. (Note that we dropped some factors of $\mathcal{S}$ from the the second line of $h_{N}$ as these will be of lower order.)

At this point, the decomposition above dealt with item one of Definition 1.4. However, one observes from the first line in the definition of $h_{N}$ that $h_{N}$ is strongly coupled to the Hamiltonian $\hat{H}_{N-1}$. We remark here that this coupled term in $h_{N}$ cannot be ignored; as one will see from Lemma 3.4, the coupled term is essential for changing the 'variance' associated with the 'Gaussian' distributions for a single spin. To deal with item two of Definition 1.4, we introduce a Gaussian process as follows.

First, define

$$
\begin{aligned}
\tilde{H}_{N}\left(\hat{\sigma}_{1}, \ldots, \hat{\sigma}_{N-1}, \sigma_{N}\right): & =\hat{H}_{N-1}\left(\hat{\sigma}_{1}, \ldots, \hat{\sigma}_{N-1}\right)+Y_{N}\left(\hat{\sigma}_{1}, \ldots, \hat{\sigma}_{N-1}, \sigma_{N}\right) \\
& +\sum_{p=2}^{\infty} \frac{\beta_{p}}{N^{(p-1) / 2}} \sum_{1 \leq i_{1}, \ldots, i_{p-1} \leq N-1} g_{i_{1}, \ldots, i_{p-1}, N} \hat{\sigma}_{i_{1}} \ldots \hat{\sigma}_{i_{p-1}} \sigma_{N} .
\end{aligned}
$$

$Y_{N}$ should satisfy the following properties:

- We desire that the following relation holds

$$
\operatorname{Cov}\left(H_{N}(\sigma), H_{N}(\rho)\right) \approx \operatorname{Cov}\left(\tilde{H}_{N}\left(\hat{\sigma}_{1}, \ldots, \hat{\sigma}_{N-1}, \sigma_{N}\right), \tilde{H}_{N}\left(\hat{\rho}_{1}, \ldots, \hat{\rho}_{N-1}, \rho_{N}\right)\right) .
$$

- We want $\hat{H}_{N-1}$ to be independent of $Y_{N}$.

- We finally want $Y_{N}$ to be independent of

$$
\sum_{p=2}^{\infty} \frac{\beta_{p}}{N^{(p-1) / 2}} \sum_{1 \leq i_{1}, \ldots, i_{p-1} \leq N-1} g_{i_{1}, \ldots, i_{p-1}, N} \hat{\sigma}_{i_{1}} \ldots \hat{\sigma}_{i_{p-1}} \sigma_{N}
$$

Broadly speaking, $Y_{N}$ is constructed via a perturbation expansion of the term containing $\mathcal{S}^{p}-1$ in $h_{N}$. This is the content of Section 2 of this paper. One then tries to compute spin distributions according to the Hamiltonian $\tilde{H}_{N}$ rather than $H_{N}$.

It is crucial to note that spin distributions according to $\tilde{H}_{N}$ and $H_{N}$ will only match if the approximation is exact

$$
\lim _{N \rightarrow \infty}\left|\operatorname{Cov}\left(\tilde{H}_{N}(\sigma), \tilde{H}_{N}(\rho)\right)-\operatorname{Cov}\left(H_{N}(\sigma), H_{N}(\rho)\right)\right|=0 .
$$


Unfortunately, there is no choice of $Y_{N}$ that does not give an error in the covariance structure of order $O(1)$. The content of Theorem 2.7 shows that the error rate of the spin distributions is of size $O\left(\frac{C^{D}}{R^{2}}\right)$, where the power $D$ appearing depends on the polynomial moment considered.

The way that this problem is circumvented is to use the fact that we can vary $R$ and $C$ as free parameters. The key observation here is that if we take $R$ far greater than any power of $C$, then the error rate in Theorem 2.7 becomes exact as $R$ goes to $\infty$. Specifically, the introduction of a finite cutoff $C$ is necessary to ensure a vanishing error rate. The crucial novelty here is the introduction of the limit process $\lim _{C \rightarrow \infty} \lim _{R \rightarrow \infty} \lim _{N \rightarrow \infty}$ in that order to make sure our approximation will give the exact spin distribution. In particular, we will observe that the process $Y_{N}$ introduced in (2.14) contains a factor that grows as $\sqrt{R^{2}+1}$. Far from causing this term to explode, there is a crucial cancellation demonstrated in Lemma 3.4.

The effect of the $R \rightarrow \infty$ limit results in a rescaling for the variance of the spin distribution. This rescaling step is what allows us to get the exact value of the constant $b_{\zeta}$ directly from the spin glass parameters. Usually, the value of $b_{\zeta}$ comes from solving some implicit optimization question. The cavity equations here provide a new and natural way to directly find the value of this variance. The justification of this limit for spin glasses with finite replica symmetry breaking is the main content of Section 3, which is the key novelty of this work. Together, Sections 2 and 3 form the majority of the work.

Another possible method used to study the spherical spin glass is the introduction of a Lagrange multiplier, such as in [19], in order to treat the sphere as a product space. These works applied large deviation estimates to relate probabilities on the sphere to probabilities using a Lagrange multiplier; on the level of the free energy, which would take the logarithm of such probabilities, these would anyway not be too much of a problem. However, these are extremely inexact if one wants the precise spin distribution. In particular, the limit procedure was proposed to exactly characterize the tail behavior of spin $\sigma_{N}$. Though the method proposed here is involved, it seems superior to the introduction of a Lagrange multiplier. Any presumable method involving a Lagrange multiplier would still require some cutoff and limit arguments to relate the tail probabilities of large values of $\sigma_{N}$ on the sphere to that of the model with the Lagrange multiplier.

The final section is dedicated to proving the stochastic process version of these results as detailed in Theorem 1.2 for models with general replica symmetry breaking. From the results of Section 3, we take limits of finite replica symmetry models to derive results for full replica symmetry breaking models. The method innovated in this paper is a pioneering way to understand the Hamiltonian of spherical spin glasses. For example, it finds a direct meaning to the constant $b$ from [19] in terms of a renormalization of the Hamiltonian instead of as a maximization procedure. We expect it has a great potential of leading to great new understanding in the future parallel to that of the Lagrange multiplier.

\section{The cavity equation for the sphere}

As we have described in the introduction, the first step to proving Theorem 1.2 is to first prove a version of the cavity equation. To this end, we need to create an auxiliary space so that the auxiliary space is represented as a product space, and so we have a natural notion of a cavity equation. 


\subsection{The construction of cavity coordinate}

Our first step in constructing the auxiliary space is to restrict the sphere so that the final $n$ coordinates lie in a compact interval. We define the restricted sphere

$$
\begin{aligned}
S_{N+n}^{C, n}=\{ & \left(\sigma_{1}, \ldots, \sigma_{N}, \sigma_{N+1}, \ldots, \sigma_{N+n}\right): \\
& \left.\sigma_{1}^{2}+\ldots+\sigma_{N}^{2}+\sigma_{N+1}^{2}+\ldots \sigma_{N+n}^{2}=N+n,\left|\sigma_{N+1}^{2}\right|, \ldots\left|\sigma_{N+n}\right| \leq C\right\},
\end{aligned}
$$

with Hamiltonian $H_{N+n}$.

We begin with the following lemma. We use the notation $\langle\cdot\rangle_{H_{N}(A)}$ to denote the expectation with respect to a Gibbs measure of the function $H_{N}$ distributed on the set $A$. When the context is obvious, we will drop mention of the Hamiltonian and simply use $\langle\cdot\rangle_{A}$ to denote the Gibbs measure on the set $A$.

Lemma 2.1. We can replace the expectation of polynomial expressions on the last $n$ coordinates as $N \rightarrow \infty$ under the Gibbs measure on $S_{N+n}$ with the expectation of the Gibbs measure over the restricted sets $S_{N+n}^{C, n}$ as we take the double limit $N \rightarrow \infty$ followed by $C \rightarrow \infty$,

$$
\begin{aligned}
& \lim _{N \rightarrow \infty} \mathbb{E}\left[\prod_{l=1}^{k}\left\langle\left(\sigma_{N+1}^{l}\right)^{e_{l, 1}} \ldots\left(\sigma_{N+n}^{l}\right)^{e_{l, n}}\right\rangle_{H_{N+n}\left(S_{N+n}\right)}\right] \\
& =\lim _{C \rightarrow \infty} \lim _{N \rightarrow \infty} \mathbb{E}\left[\prod_{l=1}^{k}\left\langle\left(\sigma_{N+1}^{l}\right)^{e_{l, 1}} \ldots\left(\sigma_{N+n}^{l}\right)^{e_{l, n}}\right\rangle_{H_{N+n}\left(S_{N+n}^{C, n}\right)}\right] .
\end{aligned}
$$

Here, the $l$ is a superscript designating the replica index while $e_{l, i}$ is the exponent computed. The exponents $e_{l, i}$ are restricted to take non-negative integer values.

Proof. The result is due to the fact that each of the spins $\sigma_{N+1}, \ldots, \sigma_{N+n}$ has subgaussian tails. We will sketch a proof in the case when $k=1$. We will let $f(\sigma)$ denote the polynomial function $\left(\sigma_{N+1}^{1}\right)^{e_{1,1}} \ldots\left(\sigma_{N+n}^{1}\right)^{e_{1, n}}$. The difference between the quantities inside the limit on the left and right hand sides can be written as

$$
-\mathbb{E} \frac{\int_{\left(S_{N+n}^{C, n}\right)^{c}} f(\sigma) e^{H_{N+n}(\sigma)} \mathrm{d} \sigma}{\int_{S_{N+n}} e^{H_{N+n}(\sigma)} \mathrm{d} \sigma}+\mathbb{E} \frac{\int_{S_{N+n}^{C, n}} f(\sigma) e^{H_{N+n}(\sigma)} \mathrm{d} \sigma \int_{\left(S_{N+n}^{C, n}\right)^{c}} e^{H_{N+n}(\sigma)} \mathrm{d} \sigma}{\int_{S_{N+n}} e^{H_{N+n}(\sigma)} \mathrm{d} \sigma \int_{S_{N+n}^{C, n}} e^{H_{N+n}(\sigma)} \mathrm{d} \sigma} .
$$

The methods used to bound both quantities are similar. We will bound the latter by two applications of the Cauchy-Schwarz inequality.

For convenience of notation, we let $\chi$ be the indicator function for the set $\left(S_{N+n}^{C, n}\right)^{c}$ in $S_{N+n}$. First of all, we see that the second quantity in (2.2) can be written and bounded as

$$
\begin{aligned}
\mathbb{E}\left[\langle f(\sigma)\rangle_{H_{N+n}\left(S_{N+n}^{C, n}\right)}\langle\chi\rangle_{H_{N+n}\left(S_{N+n}\right)}\right] & \leq\left(\mathbb{E}\left[\left(\langle f(\sigma)\rangle_{H_{N+n}\left(S_{N+n}^{C, n}\right)}\right)^{2}\right] \mathbb{E}\left[\left(\langle\chi\rangle_{H_{N+n}\left(S_{N+n}\right)}\right)^{2}\right]\right)^{1 / 2} \\
& \leq\left(\mathbb{E}\left[\left\langle f(\sigma)^{2}\right\rangle_{H_{N+n}\left(S_{N+n}^{C, n}\right)}\right] \mathbb{E}\left[\langle\chi\rangle_{H_{N+n}\left(S_{N+n}\right)}\right]\right)^{1 / 2} .
\end{aligned}
$$

The first application of the Cauchy-Schwarz inequality splits the outer expectation. The second application of the Cauchy-Schwarz inequality applies to the Gibbs measures inside the expectation. Observe that since $\chi$ is an indicator function, we have $\chi^{2}=\chi$.

To bound $\mathbb{E}\left[\left\langle f(\sigma)^{2}\right\rangle_{H_{N+n}\left(S_{N+n}^{C, n}\right)}\right]$, the only thing that has to be remarked is the following. Since, in $S_{N+n}^{C, n}$, all spins are bounded by $C$, this expectation can be bounded by a polynomial function of $C$, say $|C|^{2 \sum_{i=1}^{n} e_{1, i}}$ that is independent of $N$. 
We let $\chi_{i}$ be the indicator function of the set on which $\left|\sigma_{N+i}\right| \geq C$. We also see that there exists some $k$, not depending on $C$, such that, for $N$ sufficiently large, we have

$$
\mathbb{E}\left[\langle\chi\rangle_{H_{N+n}\left(S_{N+n}\right)}\right] \leq \sum_{i=1}^{n} \mathbb{E}\left[\left\langle\chi_{i}\right\rangle_{H_{N+n}\left(S_{N+n}\right)}\right] \leq n e^{-k C^{2}} .
$$

This is due to the fact that the spins $\sigma_{N+i}$ are subgaussian. (The distribution of a single spin is uniform on the sphere due to the rotational invariance of the Hamiltonian.)

Together, we can bound the product appearing in the last line of (2.3) by

$$
\left(n e^{-k C^{2}}|C|^{2 \sum_{i=1}^{n} e_{1, i}}\right)^{1 / 2},
$$

for $N$ sufficiently large. This will also be a bound on the difference as we take the interior limit, $N \rightarrow \infty$, on both sides. Finally, taking the exterior limit, $C \rightarrow \infty$, will bound this difference by 0 . This establishes the lemma.

In order to construct the auxiliary Hamiltonian, we would like to consider $S_{N+n}^{C, n}$ as a product set. We define the set $S_{N+n}^{C, R, n}$ as

$$
S_{N+n}^{C, R, n}=\left\{\left(\hat{\sigma}_{1}, \ldots, \hat{\sigma}_{N}\right): \hat{\sigma}_{1}^{2}+\ldots+\hat{\sigma}_{N}^{2}=N-n R^{2}\right\} \times\left\{\left|\hat{\sigma}_{N+1}\right|, \ldots,\left|\hat{\sigma}_{N+n}\right| \leq C\right\} .
$$

The map between $S_{N+n}^{C, n}$ and $S_{N+n}^{C, R, n}$ is as follows:

$$
\hat{\sigma}_{i}:=\frac{\sigma_{i} \sqrt{N-n R^{2}}}{\sqrt{N+n-\sigma_{N+1}^{2}-\ldots-\sigma_{N+n}^{2}}}, 1 \leq i \leq N,
$$

and $\hat{\sigma}_{N+i}:=\sigma_{N+i}$ for $1 \leq i \leq n$. We will denote the factor $\frac{\sqrt{N+n-\sigma_{N+1}^{2}-\ldots-\sigma_{N+n}^{2}}}{\sqrt{N-n R^{2}}}$ as $\mathcal{S}$. (This will allow us to write the fundamental expression $\sigma_{i}=\mathcal{S} \hat{\sigma}_{i}$ for $1 \leq i \leq N$.) Notice that the first part of the decomposition of $S_{N+n}^{C, R, n}$ is the sphere $S_{N}\left(N-n R^{2}\right)$.

When defined in terms of the new variables $\hat{\sigma}_{i}$, we will be able to write the Hamiltonian $H_{N+n}$ on the set $S_{N+n}^{C, n}$ as the following Hamiltonian on the set $S_{N+n}^{C, R, n}$

$$
H_{N+n}\left(\hat{\sigma}_{1}, \ldots, \hat{\sigma}_{N+n}\right)=\sum_{p=2}^{\infty} \beta_{p} \frac{1}{(N+n)^{(p-1) / 2}} \sum_{k=0}^{n} \sum_{\mathcal{I}_{k}} g_{i_{1}, i_{2}, \ldots, i_{p}} \mathcal{S}^{p-k} \hat{\sigma}_{i_{1}} \ldots \hat{\sigma}_{i_{p}},
$$

where $\mathcal{I}_{k}:=\left\{\left(i_{1}, \ldots, i_{p}\right):\left|\left\{i_{1}, \ldots, i_{p}\right\} \cap\{N+1, \ldots, N+n\}\right|=k\right\}$.

Remark 2.2. We remark here that the cardinality is counted with multiplicity. For example, the choice $\left(i_{1}, i_{2}, i_{3}, \ldots, i_{p}\right)=(N+1, N+1,1,1, \ldots, 1)$ with the last $p-2$ entries being 1 would be in the set $\mathcal{I}_{2}$. Additionally, $\mathcal{I}_{0}$ would only involve the coordinates $\hat{\sigma}_{1}, \ldots, \hat{\sigma}_{N}$ and none of the cavity coordinates.

The change of variables makes us consider slightly different test functions.

Lemma 2.3. The following equality holds for fixed $C$ and $\left\{e_{l, n}\right\}$ a collection of nonnegative integers. $R$ can be freely chosen,

$$
\begin{aligned}
\lim _{N \rightarrow \infty} \mathbb{E}\left[\prod_{l=1}^{k}\left\langle\prod_{j=1}^{n}\left(\sigma_{N+j}^{l}\right)^{e_{l, j}}\right\rangle_{H_{N+n}\left(S_{N+n}^{C, n}\right)}\right] \\
=\lim _{N \rightarrow \infty} \mathbb{E}\left[\prod_{l=1}^{k} \frac{\int_{S_{N+n}^{C, R, n}} \prod_{j=1}^{n}\left(\hat{\sigma}_{N+j}^{l}\right)^{e_{l . j}} e^{\frac{-\left(\hat{\sigma}_{N+j}^{l}\right)^{2}}{2}} e^{H_{N+n}(\hat{\sigma})} \mathrm{d} \hat{\sigma}}{\int_{S_{N+n}^{C, R, n}} \prod_{j=1}^{n} e^{-\frac{\left(\hat{\sigma}_{N+j}^{l}\right)^{2}}{2}} e^{H_{N+n}(\hat{\sigma})} \mathrm{d} \hat{\sigma}}\right] .
\end{aligned}
$$


Proof. The proof will follow when we investigate the change in coordinates. We will illustrate the details when $k=1$. The general proof is similar.

Applying the change of coordinates $\sigma_{k} \rightarrow \hat{\sigma}_{k}$ for $k$ between 1 and $N+n$ to the right hand side of (2.6), we see that

$$
\begin{aligned}
& \lim _{N \rightarrow \infty} \mathbb{E}\left[\frac{\int_{S_{N+n}^{C, n}} \prod_{j=1}^{n} \sigma_{N+j}^{e_{1, j}} e^{H_{N+n}(\sigma)} \mathrm{d} \sigma}{\int_{S_{N+n}^{C, n}} e^{H_{N+n}(\sigma)} \mathrm{d} \sigma}\right] \\
& =\lim _{N \rightarrow \infty} \mathbb{E}\left[\frac{\int_{S_{N+n}^{C, R}, n} \prod_{j=1}^{n} \hat{\sigma}_{N+j}^{e_{1, j}} e^{H_{N+n}(\hat{\sigma})} \mathcal{S}^{N} \mathrm{~d} \hat{\sigma}}{\int_{S_{N+n}^{C, R}, n} e^{H_{N+n}(\hat{\sigma})} \mathcal{S}^{N} \mathrm{~d} \hat{\sigma}}\right] .
\end{aligned}
$$

The main difference is that we have introduced the Jacobian factor $\mathcal{S}^{N}$ on the numerator and the denominator.

We notice that

$$
\lim _{N \rightarrow \infty} \mathcal{S}^{N}=\lim _{N \rightarrow \infty}\left(1+\frac{n R^{2}+n-\hat{\sigma}_{N+1}^{2}-\ldots-\hat{\sigma}_{N+n}^{2}}{N-n R^{2}}\right)^{\frac{N}{2}}=e^{\frac{n R^{2}+n-\sum_{i=1}^{n} \hat{\sigma}_{N+i}^{2}}{2}},
$$

and this approach is uniform when we restrict $\left|\hat{\sigma}_{N+i}\right| \leq C$.

We can now apply the Exchange Lemma 2.4 in order to justify

$$
\begin{aligned}
\lim _{N \rightarrow \infty} & \mathbb{E}\left[\frac{\int_{S_{N+n}^{C, R, n}} \prod_{j=1}^{n} \hat{\sigma}_{N+j}^{e_{1, j}} e^{H_{N+n}(\hat{\sigma})} \mathcal{S}^{N} \mathrm{~d} \hat{\sigma}}{\int_{S_{N+n}^{C, R, n}} e^{H_{N+n}(\hat{\sigma})} \mathcal{S}^{N} \mathrm{~d} \hat{\sigma}}\right] \\
= & \lim _{N \rightarrow \infty} \mathbb{E}\left[\frac{\int_{S_{N+n}^{C, R, n}} e^{n R^{2}+n} \prod_{j=1}^{n} \hat{\sigma}_{N+j}^{e_{1, j}} e^{-\frac{\hat{\sigma}_{N+j}^{2}}{2}} e^{H_{N+n}(\hat{\sigma})} \mathrm{d} \hat{\sigma}}{\int_{S_{N+n}^{C, R, n}} e^{n R^{2}+n} \prod_{j=1}^{n} e^{-\frac{\hat{\sigma}_{N+j}^{2}}{2}} e^{H_{N+n}(\hat{\sigma})} \mathrm{d} \hat{\sigma}}\right] .
\end{aligned}
$$

We can cancel the factor of $e^{n R^{2}+n}$ appearing in both the numerator and denominator.

Lemma 2.4 (Exchange Lemma). Consider a random Hamiltonian $H_{N}$ on some domain $D$. Assume there exists some constant $K$ such that all of $\left|f_{N}\right|,\left|g_{N}\right|,\left|\hat{f}_{N}\right|$ and $\left|\hat{g}_{N}\right|$ are bounded by $K$ on the domain $D$ and that $g_{N}$ and $\hat{g}_{N}$ are positive and bounded from below by $K^{-1}$. Assume in addition that $\lim _{N \rightarrow \infty}\left|\hat{f}_{N}-f_{N}\right|,\left|\hat{g}_{N}-g_{N}\right|=0$ uniformly on $D$.

Then, the following equality holds provided at least one of the sides is known to exist.

$$
\lim _{N \rightarrow \infty} \mathbb{E}\left[\frac{\int_{D} f_{N} e^{H_{N}} d \sigma}{\int_{D} g_{N} e^{H_{N}} d \sigma}\right]=\lim _{N \rightarrow \infty} \mathbb{E}\left[\frac{\int_{D} \hat{f}_{N} e^{H_{N}} d \sigma}{\int_{D} \hat{g}_{N} e^{H_{N}} d \sigma}\right]
$$

Proof. We can write the difference of the expressions at finite $N$ as,

$$
\begin{aligned}
& \left|\mathbb{E}\left[\frac{\int_{D}\left(f_{N}-\hat{f}_{N}\right) e^{H_{N}} \mathrm{~d} \sigma \int_{D} \hat{g}_{N} e^{H_{N}} \mathrm{~d} \sigma}{\int_{D} g_{N} e^{H_{N}} \mathrm{~d} \sigma \int_{D} \hat{g}_{N} e^{H_{N}} \mathrm{~d} \sigma}\right]-\mathbb{E}\left[\frac{\int_{D}\left(g_{N}-\hat{g}_{N}\right) e^{H_{N}} \mathrm{~d} \sigma \int_{D} \hat{f}_{N} e^{H_{N}} \mathrm{~d} \sigma}{\int_{D} g_{N} e^{H_{N}} \mathrm{~d} \sigma \int_{D} \hat{g}_{N} e^{H_{N}} \mathrm{~d} \sigma}\right]\right| \\
& \leq \mathbb{E}\left[\frac{\int_{D}\left|f_{N}-\hat{f}_{N}\right| e^{H_{N}} \mathrm{~d} \sigma \int_{D}\left|\hat{g}_{N}\right| e^{H_{N}} \mathrm{~d} \sigma}{\int_{D} g_{N} e^{H_{N}} \mathrm{~d} \sigma \int_{D} \hat{g}_{N} e^{H_{N}} \mathrm{~d} \sigma}\right]+\mathbb{E}\left[\frac{\int_{D}\left|g_{N}-\hat{g}_{N}\right| e^{H_{N}} \mathrm{~d} \sigma \int_{D}\left|\hat{f}_{N}\right| e^{H_{N}} \mathrm{~d} \sigma}{\int_{D} g_{N} e^{H_{N}} \mathrm{~d} \sigma \int_{D} \hat{g}_{N} e^{H_{N}} \mathrm{~d} \sigma}\right] \\
& \leq \mathbb{E}\left[\frac{\int_{D}\left|f_{N}-\hat{f}_{N}\right| e^{H_{N}} \mathrm{~d} \sigma \int_{D} K e^{H_{N}} \mathrm{~d} \sigma}{\int_{D} K^{-1} e^{H_{N}} \mathrm{~d} \sigma \int_{D} K^{-1} e^{H_{N}} \mathrm{~d} \sigma}\right]+\mathbb{E}\left[\frac{\int_{D}\left|g_{N}-\hat{g}_{N}\right| e^{H_{N}} \mathrm{~d} \sigma \int_{D} K e^{H_{N}} \mathrm{~d} \sigma}{\int_{D} K^{-1} e^{H_{N}} \mathrm{~d} \sigma \int_{D} K^{-1} e^{H_{N}} \mathrm{~d} \sigma}\right] \\
& \leq K^{3} \mathbb{E}\left[\frac{\int_{D}\left|f_{N}-\hat{f}_{N}\right| e^{H_{N}} \mathrm{~d} \sigma}{\int_{D} e^{H_{N}} \mathrm{~d} \sigma}\right]+K^{3} \mathbb{E}\left[\frac{\int_{D}\left|g_{N}-\hat{g}_{N}\right| e^{H_{N}} \mathrm{~d} \sigma}{\int_{D} e^{H_{N}} \mathrm{~d} \sigma}\right] .
\end{aligned}
$$


Noting our limiting condition on the differences $\left|f_{N}-\hat{f}_{N}\right|$ and $\left|g_{N}-\hat{g}_{N}\right|$, we can show that as we take $N$ to $\infty$, the difference on the last line of (2.10) will go to 0 . This establishes the existence of the limit on both sides as long as one of them exists as well as their equality.

\subsection{Construction of the cavity Hamiltonian}

Recall the Hamiltonian (2.5) on $S_{N+n}^{C, R, n}$.

Ideally, we would like to write the Hamiltonian as a Hamiltonian on the sphere $S_{N}\left(N-n R^{2}\right)$ plus a perturbation term. Namely, we have

$$
\begin{aligned}
& H_{N+n}\left(\hat{\sigma}_{1}, \ldots, \hat{\sigma}_{N+n}\right)=\sum_{p=2}^{\infty} \beta_{p} \frac{1}{(N+n)^{(p-1) / 2}} \sum_{\mathcal{I}_{0}} g_{i_{1}, i_{2}, \ldots, i_{p}} \hat{\sigma}_{i_{1}} \ldots \hat{\sigma}_{i_{p}} \\
& +\sum_{p=2}^{\infty} \beta_{p} \frac{1}{(N+n)^{(p-1) / 2}} \sum_{\mathcal{I}_{0}} g_{i_{1}, i_{2}, \ldots, i_{p}} \hat{\sigma}_{i_{1}} \ldots \hat{\sigma}_{i_{p}}\left(\mathcal{S}^{p}-1\right) \\
& +\sum_{p=2}^{\infty} \beta_{p} \frac{1}{(N+n)^{(p-1) / 2}} \sum_{\mathcal{I}_{1}} g_{i_{1}, i_{2}, \ldots, i_{p}} \hat{\sigma}_{i_{1}} \ldots \hat{\sigma}_{i_{p}}\left(\mathcal{S}^{p-1}\right) \\
& +\sum_{p=2}^{\infty} \beta_{p} \frac{1}{(N+n)^{(p-1) / 2}} \sum_{k=2}^{n} \sum_{\mathcal{I}_{k}} g_{i_{1}, i_{2}, \ldots, i_{p}} \hat{\sigma}_{i_{1}} \ldots \hat{\sigma}_{i_{p}}\left(\mathcal{S}^{p-k}\right) .
\end{aligned}
$$

We make some remarks about the above decomposition. The term on the first line is a function only of the spins $\hat{\sigma}_{1}, \ldots, \hat{\sigma}_{N}$. We may be able to interpret this as a Hamiltonian defined on the sphere $S_{N}\left(N-n R^{2}\right)$. We remark also that it is of the highest order $N$ in variance. The term on the second line will be a term of order 1 in variance, but it is coupled to the term on the first line.

The terms on the third line are additionally of order 1 in variance and independent of the terms on the first two; notice that each of these terms will only involve exactly 1 of the cavity coordinates $\hat{\sigma}_{N+1}, \ldots, \hat{\sigma}_{N+n}$.

The terms on the fourth line are independent of those that have come before and have variance of order $1 / N$. We will be able to treat these terms as errors.

The issues that arise in computing distributional equivalences come from the second term in the above expression; it is coupled with the main Hamiltonian on the sphere $S_{N}\left(N-n R^{2}\right)$. In order to perform computations in the future, what we would like to do is instead replace this term with one that is independent of the main Hamiltonian on the first line. Namely, we would like to consider

$$
\begin{aligned}
& \tilde{H}_{N+n}\left(\hat{\sigma}_{1}, \ldots, \hat{\sigma}_{N+n}\right)=\sum_{p=2}^{\infty} \beta_{p} \frac{1}{(N+n)^{(p-1) / 2}} \sum_{\mathcal{I}_{0}} g_{i_{1}, i_{2}, \ldots, i_{p}} \hat{\sigma}_{i_{1}} \ldots \hat{\sigma}_{i_{p}} \\
& +\sum_{p=2}^{\infty} \sqrt{p} \beta_{p} \frac{1}{(N+n)^{(p-1) / 2}} \sum_{\mathcal{I}_{0}} \sum_{i=1}^{n} g_{i_{1}, i_{2}, \ldots, i_{p}}^{N+i} \frac{\sqrt{R^{2}+1}-\frac{\hat{\sigma}_{N+i}^{2}}{2 \sqrt{R^{2}+1}}}{\sqrt{N+n}} \hat{\sigma}_{i_{1}} \ldots \hat{\sigma}_{i_{p}} \\
& +\sum_{p=2}^{\infty} \beta_{p} \frac{1}{(N+n)^{(p-1) / 2}} \sum_{\mathcal{I}_{1}} g_{i_{1}, i_{2}, \ldots, i_{p}} \hat{\sigma}_{i_{1}} \ldots \hat{\sigma}_{i_{p}},
\end{aligned}
$$

where the $g_{i_{1}, \ldots, i_{p}}^{N+i}$ are $N(0,1)$ Gaussian random variables independent of all other randomness. Notice also that in the local field(magnetization) term on the last line, we have dropped the rescaling term $\mathcal{S}^{p-1}$. We will eventually be able to show, provided sufficient decay of the $\beta_{p}$, that $\mathcal{S}^{p-1}-1$ will lead to a term that is of smaller order. Along the same line, we have removed the fourth line of error terms, anticipating that they will eventually be shown to be insignificant. 
For simplicity of notation, we will denote

$$
\begin{aligned}
\hat{H}_{N}(\hat{\sigma}) & :=\sum_{p=2}^{\infty} \beta_{p} \frac{1}{(N+n)^{(p-1) / 2}} \sum_{1 \leq i_{1} \ldots i_{p} \leq N} g_{i_{1}, i_{2}, \ldots, i_{p}} \hat{\sigma}_{i_{1}} \ldots \hat{\sigma}_{i_{p}}, \\
Y^{i}(\hat{\sigma}) & :=\sum_{p=2}^{\infty} \sqrt{p} \beta_{p} \frac{1}{(N+n)^{p / 2}} \sum_{1 \leq i_{1}, \ldots, i_{p} \leq N} g_{i_{1}, \ldots, i_{p}}^{N+i} \hat{\sigma}_{i_{1}} \ldots \hat{\sigma}_{i_{p}}, \\
Z^{i}(\hat{\sigma}) & :=\sum_{p=2}^{\infty} \beta_{p} \frac{1}{(N+n)^{(p-1) / 2}} \sum_{\mathcal{I}_{1}^{i}} g_{i_{1}, \ldots, i_{p}} \prod_{k: i_{k} \neq N+i} \hat{\sigma}_{i_{k}} .
\end{aligned}
$$

where we define $\mathcal{I}_{1}^{i}:=\mathcal{I}_{1} \cap\left\{i_{1}, \ldots, i_{p}:\left\{i_{1}, \ldots, i_{p}\right\} \cap\{N+1, \ldots, N+n\}=\{N+i\}\right\}$. We remark here that exactly one of the $\left\{i_{1}, \ldots, i_{p}\right\}$ belongs to the set $\{N+1, \ldots, N+n\}$, and this entry must be $N+i$. This is a shorthand for the three lines in the decomposition of (2.12).

In order to justify the replacement of the Gaussian process $H_{N+n}$ with $\tilde{H}_{N+n}$, we compare the variances for the two processes.

\subsection{A perturbative expression for the correlation structure of $H_{N+n}$}

Our goal in this subsection is to find a perturbative expression for the covariance structure of $H_{N+n}$ in terms of the newly introduced coordinates $\hat{\sigma}$ and $\hat{\rho}$.

Remark 2.5. We remark that, for our later computation, it is only necessary to know the covariance structure up to the leading order in $N$.

In addition, our limiting procedure will first take the $N \rightarrow \infty$ limit before any operations are taken on $C$ or $R$. In the computations that follow, any term of subleading order in $N$ may have coefficients that depend on $C$ or $R$. These can be safely ignored as we take $N \rightarrow \infty$ first. Such prefactors for subleading terms will not be explicitly written. Only leading order terms involving $R$ or $C$ will have to be written out.

The covariance structure of $H_{N+n}$ is given as follows,

$$
\operatorname{Cov}\left(H_{N+n}(\sigma), H_{N+n}(\rho)\right)=(N+n) \sum_{p=2}^{\left\lfloor N^{\alpha}\right\rfloor} \beta_{p}^{2} R_{\sigma, \rho}^{p}+(N+n) \sum_{p=\left\lfloor N^{\alpha}\right\rfloor+1}^{\infty} \beta_{p}^{2} R_{\sigma, \rho}^{p}
$$

where, as a reminder, $R_{\sigma, \rho}=\frac{1}{N+n} \sum_{i=1}^{N+n} \sigma_{i} \rho_{i}$.

We have split the summation in such a way because we will apply a first order perturbation argument to the sum over the smaller $p$ while we apply a trivial bound to the larger $p$. In the calculations that follow, $K$ will be a constant that may differ from line to line.

For the sum over the larger $p$, we apply our Assumption 1.1 to bound each $\beta_{p} \leq$ $K p^{-3 / 2-\epsilon}$. We see that we obtain,

$$
(N+n) \sum_{p=\left\lfloor N^{\alpha}\right\rfloor+1}^{\infty} \beta_{p}^{2} R_{\sigma, \rho}^{p} \leq(N+n) \sum_{p=\left\lfloor N^{\alpha}\right\rfloor+1}^{\infty} \frac{K}{p^{3+2 \epsilon}} \leq(N+n) \frac{K}{N^{(2+2 \epsilon) \alpha}} \leq K N^{1-(2+2 \epsilon) \alpha} .
$$

For the smaller powers of $p$, we have to perform a perturbation expansion. By an exercise in calculus, we see that if $x$ is an order $\frac{1}{N}$ positive quantity and $p \leq N^{\alpha} \ll N$, then we have the inequality

$$
(1+x)^{p} \leq 1+p x+2 p^{2} x^{2} \leq 1+p x+O\left(N^{2 \alpha-2}\right) .
$$


Using this identity, we can first separate the terms involving the spins $\sigma_{N+i} \rho_{N+i}$ for $i$ between 1 and $n$. Notice that we have, for $p \leq N^{\alpha} \ll N$,

$$
\begin{aligned}
\left(\frac{1}{N+n} \sum_{i=1}^{N+n} \sigma_{i} \rho_{i}\right)^{p} & =\left(\frac{1}{N+n} \sum_{i=1}^{N} \sigma_{i} \rho_{i}\right)^{p} \\
& +p\left(\frac{1}{N+n} \sum_{i=1}^{N} \sigma_{i} \rho_{i}\right)^{p-1}\left(\frac{1}{N+n} \sum_{i=1}^{n} \sigma_{N+i} \rho_{N+i}\right)+O\left(N^{2 \alpha-2}\right)
\end{aligned}
$$

Now we replace the spins $\sigma_{i}, \rho_{i}$ with the rescaled coordinates $\hat{\sigma}_{i}, \hat{\rho}_{i}$. As mentioned previously, this would require a perturbation analysis of $\mathcal{S}^{p}$.

Applying this expansion to the $p$ th power of the overlaps for $p \leq N^{\alpha} \ll N$, we have

$$
\begin{aligned}
& \beta_{p}^{2}\left(\frac{1}{N+n} \sum_{i=1}^{N} \sigma_{i} \rho_{i}\right)^{p} \\
& =\beta_{p}^{2}\left(\frac{1}{N+n} \sum_{i=1}^{N} \hat{\sigma}_{i} \hat{\rho}_{i}\right)^{p}\left(1+\frac{n R^{2}+n-\hat{\sigma}_{N+1}^{2}-\ldots \hat{\sigma}_{N+n}^{2}}{N-n R^{2}}\right)^{p / 2} \\
& \quad\left(1+\frac{n R^{2}+n-\hat{\rho}_{N+1}^{2}-\ldots \hat{\rho}_{N+n}^{2}}{N-n R^{2}}\right)^{p / 2} \\
& =\beta_{p}^{2} R_{\hat{\sigma}, \hat{\rho}}^{p}+\frac{p \beta_{p}^{2}}{2} R_{\hat{\sigma}, \hat{\rho}}^{p} \frac{n R^{2}+n-\hat{\sigma}_{N+1}^{2}-\ldots \hat{\sigma}_{N+n}^{2}}{N+n} \\
& \quad+\frac{p \beta_{p}^{2}}{2} R_{\hat{\sigma}, \hat{\rho}}^{p} \frac{n R^{2}+n-\hat{\rho}_{N+1}^{2}-\ldots \hat{\rho}_{N+n}^{2}}{N+n}+O\left(\beta_{p}^{2} N^{2 \alpha-2}\right),
\end{aligned}
$$

where we define the terms

$$
R_{\hat{\sigma}, \hat{\rho}}=\frac{1}{N+n} \sum_{i=1}^{N} \hat{\sigma}_{i} \hat{\rho}_{i} .
$$

We also have the similar inequality $(1+x)^{p} \leq 1+2 p x \leq 1+O\left(N^{\alpha-1}\right)$ for $x=O\left(N^{-1}\right)$ and $p \leq N^{\alpha} \ll N$. Thus, we observe that, for $p \leq N^{\alpha} \ll N$, we have

$$
\begin{aligned}
& p \beta_{p}^{2}\left(\frac{1}{N+n} \sum_{i=1}^{N} \sigma_{i} \rho_{i}\right)^{p-1}\left(\frac{1}{N+n} \sum_{i=1}^{n} \sigma_{N+i} \rho_{N+i}\right) \\
& =p \beta_{p}^{2}\left(\frac{1}{N+n} \sum_{i=1}^{N} \hat{\sigma}_{i} \hat{\rho}_{i}\right)^{p-1}\left(\frac{1}{N+n} \sum_{i=1}^{n} \hat{\sigma}_{N+i} \hat{\rho}_{N+i}\right)+O\left(p \beta_{p}^{2} N^{\alpha-2}\right) .
\end{aligned}
$$

Combining the previous perturbation estimates, we derive the following expression for the covariance.

$$
\begin{aligned}
& \operatorname{Cov}\left(H_{N+n}(\sigma), H_{N+n}(\rho)\right)= \\
& (N+n) \sum_{p=2}^{\left\lfloor N^{\alpha}\right\rfloor} \beta_{p}^{2} R_{\hat{\sigma}, \hat{\rho}}^{p}+\sum_{p=2}^{\left\lfloor N^{\alpha}\right\rfloor} \frac{p}{2} \beta_{p}^{2}\left(n R^{2}+n-\hat{\sigma}_{N+1}^{2}-\ldots-\hat{\sigma}_{N+n}^{2}\right) R_{\hat{\sigma}, \hat{\rho}}^{p} \\
& +\sum_{p=2}^{\left\lfloor N^{\alpha}\right\rfloor} \frac{p}{2} \beta_{p}^{2}\left(n R^{2}+n-\hat{\rho}_{N+1}^{2}-\ldots-\hat{\rho}_{N+n}^{2}\right) R_{\hat{\sigma}, \hat{\rho}}^{p}+\sum_{p=2}^{\left\lfloor N^{\alpha}\right\rfloor} p \beta_{p}^{2} R_{\hat{\sigma}, \hat{\rho}}^{p-1}\left(\sum_{i=1}^{n} \hat{\sigma}_{N+i} \hat{\rho}_{N+i}\right) \\
& +\sum_{p=2}^{\left\lfloor N^{\alpha}\right\rfloor}\left[O\left(\beta_{p}^{2} N^{2 \alpha-1}\right)+O\left(p \beta_{p}^{2} N^{\alpha-1}\right)\right]+O\left(N^{1-(2+2 \epsilon) \alpha}\right) .
\end{aligned}
$$


We see that if we choose any value of $\alpha$ between $\frac{1}{2+2 \epsilon}$ and $\frac{1}{2}$ and apply the summability of the series $\beta_{p}^{2}$ and $p \beta_{p}^{2}$, we will be able to show that the error is $o(1)$, which will be good enough for later purposes.

Now, we have to replace the restricted summation up to the value of $p=\left\lfloor N^{\alpha}\right\rfloor$ with the sum to $\infty$. We will apply trivial bounds to these terms using $R_{\hat{\sigma}, \hat{\rho}} \leq 1$. Observe that we have

$$
\begin{aligned}
& (N+n) \sum_{p=\left\lfloor N^{\alpha}\right\rfloor}^{\infty} \beta_{p}^{2} R_{\hat{\sigma}, \hat{\rho}}^{p}+\sum_{p=\left\lfloor N^{\alpha}\right\rfloor}^{\infty} \frac{p}{2} \beta_{p}^{2}\left(n R^{2}+n-\hat{\sigma}_{N+1}^{2}-\ldots-\hat{\sigma}_{N+n}^{2}\right) R_{\hat{\sigma}, \hat{\rho}}^{p} \\
& +\sum_{p=\left\lfloor N^{\alpha}\right\rfloor}^{\infty} \frac{p}{2} \beta_{p}^{2}\left(n R^{2}+n-\hat{\rho}_{N+1}^{2}-\ldots-\hat{\rho}_{N+n}^{2}\right) R_{\hat{\sigma}, \hat{\rho}}^{p} \\
& +\sum_{p=\left\lfloor N^{\alpha}\right\rfloor}^{\infty} p \beta_{p}^{2} R_{\hat{\sigma}, \hat{\rho}}^{p-1}\left(\sum_{i=1}^{n} \hat{\sigma}_{N+i} \hat{\rho}_{N+i}\right) \\
& \leq(N+n) \sum_{p=\left\lfloor N^{\alpha}\right\rfloor}^{\infty} \frac{K}{p^{3+2 \epsilon}}+\sum_{p=\left\lfloor N^{\alpha}\right\rfloor}^{\infty} \frac{K}{p^{2+2 \epsilon}} \leq O\left(N^{1-(2+2 \epsilon) \alpha}\right)+O\left(N^{-(1+2 \epsilon) \alpha}\right) .
\end{aligned}
$$

For a choice of $\alpha \leq \frac{1}{2}$ and $\geq \frac{1}{2+2 \epsilon}$, as before, this will be an error term.

Finally, we derive the expression,

$$
\begin{aligned}
& \operatorname{Cov}\left(H_{N+n}(\sigma), H_{N+n}(\rho)\right)= \\
& (N+n) \sum_{p=2}^{\infty} \beta_{p}^{2} R_{\hat{\sigma}, \hat{\rho}}^{p}+\sum_{p=2}^{\infty} \frac{p}{2} \beta_{p}^{2}\left(n R^{2}+n-\hat{\sigma}_{N+1}^{2}-\ldots-\hat{\sigma}_{N+n}^{2}\right) R_{\hat{\sigma}, \hat{\rho}}^{p} \\
& +\sum_{p=2}^{\infty} \frac{p}{2} \beta_{p}^{2}\left(n R^{2}+n-\hat{\rho}_{N+1}^{2}-\ldots-\hat{\rho}_{N+n}^{2}\right) R_{\hat{\sigma}, \hat{\rho}}^{p} \\
& +\sum_{p=2}^{\infty} p \beta_{p}^{2} R_{\hat{\sigma}, \hat{\rho}}^{p-1}\left(\sum_{i=1}^{n} \hat{\sigma}_{N+i} \hat{\rho}_{N+i}\right)+o(1) .
\end{aligned}
$$

\subsection{The Covariance structure of $\tilde{H}_{N}$}

Recall that in the expression (2.12), we have separated the Hamiltonian $\tilde{H}_{N}$ into three independent parts. Namely, we have the decomposition,

$$
\begin{aligned}
\tilde{H}_{N}\left(\hat{\sigma}_{1}, \ldots, \hat{\sigma}_{N+n}\right) & =\hat{H}_{N}\left(\hat{\sigma}_{1}, \ldots, \hat{\sigma}_{N}\right)+\sum_{i=1}^{n} Y^{i}\left(\hat{\sigma}_{1}, \ldots, \hat{\sigma}_{N}\right)\left(\sqrt{R^{2}+1}-\frac{\hat{\sigma}_{N+i}^{2}}{2 \sqrt{R^{2}+1}}\right) \\
& +\sum_{i=1}^{n} Z^{i}\left(\hat{\sigma}_{1}, \ldots, \hat{\sigma}_{N}\right) \hat{\sigma}_{N+i} .
\end{aligned}
$$

The three parts $\hat{H}_{N}, Y^{i}$, and $Z^{i}$ are independent of each other. We compute the covariance of each part separately.

Observe,

$$
\operatorname{Cov}\left(\hat{H}_{N}\left(\hat{\sigma}_{1}, \ldots, \hat{\sigma}_{N}\right), \hat{H}_{N}\left(\hat{\rho}_{1}, \ldots, \hat{\rho}_{N}\right)\right)=(N+n) \sum_{p=2}^{\infty} \beta_{p}^{2} R_{\hat{\sigma}, \hat{\rho}}^{p}
$$


Also,

$$
\begin{aligned}
& \operatorname{Cov}\left(Y^{i}(\hat{\sigma})\left(\sqrt{R^{2}+1}-\frac{\hat{\sigma}_{N+i}^{2}}{2 \sqrt{R^{2}+1}}\right), Y^{j}(\hat{\rho})\left(\sqrt{R^{2}+1}-\frac{\hat{\rho}_{N+j}^{2}}{2 \sqrt{R^{2}+1}}\right)\right) \\
& =\delta_{i, j}\left(\sqrt{R^{2}+1}-\frac{\hat{\sigma}_{N+i}^{2}}{2 \sqrt{R^{2}+1}}\right)\left(\sqrt{R^{2}+1}-\frac{\hat{\rho}_{N+j}^{2}}{2 \sqrt{R^{2}+1}}\right) \sum_{p=2}^{\infty} p \beta_{p}^{2} R_{\hat{\sigma}, \hat{\rho}}^{p} \\
& =\delta_{i, j}\left(R^{2}+1-\frac{1}{2} \hat{\sigma}_{N+i}^{2}-\frac{1}{2} \hat{\rho}_{N+j}^{2}+\frac{\hat{\sigma}_{N+i}^{2} \hat{\rho}_{N+j}^{2}}{4\left(R^{2}+1\right)}\right) \sum_{p=2}^{\infty} p \beta_{p}^{2} R_{\hat{\sigma}, \hat{\rho}}^{p} .
\end{aligned}
$$

Finally,

$\operatorname{Cov}\left(Z^{i}\left(\hat{\sigma}_{1}, \ldots, \hat{\sigma}_{N}\right) \hat{\sigma}_{N+i}, Z^{j}\left(\hat{\rho}_{1}, \ldots, \hat{\rho}_{N}\right) \hat{\rho}_{N+j}\right)=\delta_{i, j} \hat{\sigma}_{N+i} \hat{\rho}_{N+j} \sum_{p=2}^{\infty} p \beta_{p}^{2} R_{\hat{\sigma}, \hat{\rho}}^{p-1}$.

We can combine all of these terms to compute the covariance of $\tilde{H}_{N+n}$.

$$
\begin{aligned}
& \operatorname{Cov}\left(\tilde{H}_{N+n}(\hat{\sigma}), \tilde{H}_{N+n}(\hat{\rho})\right)=(N+n) \sum_{p=2}^{\infty} \beta_{p}^{2} R_{\hat{\sigma}, \hat{\rho}}^{p}+\sum_{p=2}^{\infty} p \beta_{p}^{2} R_{\hat{\sigma}, \hat{\rho}}^{p-1}\left(\sum_{i=1}^{n} \hat{\sigma}_{N+i} \hat{\rho}_{N+i}\right) \\
& \quad+\sum_{p=2}^{\infty} \frac{p}{2} \beta_{p}^{2} R_{\hat{\sigma}, \hat{\rho}}^{p}\left(n R^{2}+n-\hat{\sigma}_{N+i}^{2}-\ldots-\hat{\sigma}_{N+n}^{2}\right) \\
& \quad+\sum_{p=2}^{\infty} \frac{p}{2} \beta_{p}^{2} R_{\hat{\sigma}, \hat{\rho}}^{p}\left(n R^{2}+n-\hat{\rho}_{N+i}^{2}-\ldots-\hat{\rho}_{N+n}^{2}\right) \\
& \quad+\sum_{p=2}^{\infty} \frac{p \beta_{p}^{2} R_{\hat{\sigma}, \hat{\rho}}^{p}}{4\left(R^{2}+1\right)}\left(\sum_{i=1}^{n} \hat{\sigma}_{N+i}^{2} \hat{\rho}_{N+i}^{2}\right) .
\end{aligned}
$$

We now compute the differences in the respective covariances. We see that

$$
\begin{aligned}
& \left|\operatorname{Cov}\left(H_{N+n}(\hat{\sigma}), H_{N+n}(\hat{\rho})\right)-\operatorname{Cov}\left(\tilde{H}_{N+n}(\hat{\sigma}), \tilde{H}_{N+n}(\hat{\rho})\right)\right|= \\
& \sum_{p=2}^{\infty} \frac{p}{2} \beta_{p}^{2} \sum_{i=1}^{n} \frac{\hat{\sigma}_{N+i}^{2} \hat{\rho}_{N+i}^{2}}{4\left(R^{2}+1\right)}+o(1) \leq \sum_{p=2}^{\infty} \frac{p}{2} \beta_{p}^{2} \frac{n C^{4}}{4\left(R^{2}+1\right)}+o(1)=\mathrm{O}\left(\frac{C^{4}}{R^{2}}\right)+o(1),
\end{aligned}
$$

holds on the domain $S_{N+n}^{C, R, n}$. The following remark gives some motivation on the choice of $Y^{i}$.

Remark 2.6. In order to proceed with any future cavity computation, it was necessary to treat the Gaussian components of the Hamiltonian $H_{N+n}$ that involve any of the extra spins $\hat{\sigma}_{N+i}$ as independent from the Hamiltonian $\hat{H}_{N} . Y^{i}$ was created to approximate the second line of (2.11).

However, the Gaussian components of the second line of (2.11) are coupled to those of the first line $\left(\hat{H}_{N}\right)$. Any choice of independent $Y^{i}$ to approximate the second line in the covariance structure will necessarily create some error as $\left|\hat{\sigma}_{N+i}\right|$ is allowed to vary between 0 and $C$. The choice of $Y^{i}$ here assures the approximation is exact at $\left|\hat{\sigma}_{i}\right|=0$.

As we shall see in the next subsection, the difference in the covariance structure quantifies the difference in the spin distributions according to the Hamiltonians $\tilde{H}$ and $H$. The spin distributions will match only if the covariance is 0 . To justify comparing the spin distributions of $\tilde{H}$ and $H$, we see that we will have to take a double limit procedure $\lim _{C \rightarrow \infty} \lim _{R \rightarrow \infty} \lim _{N \rightarrow \infty}$ in that order. 


\subsection{Comparison of the modified Cavity Hamiltonian}

What we will show now is that the expectation of quantities computed with respect to the Hamiltonian $\tilde{H}_{N+n}$ over the restricted sphere $S_{N+n}^{C, R, n}$ will have a small difference from the same quantity computed with respect to the Hamiltonian $H_{N+n}$ via an interpolation procedure. We can then proceed to compute spin distributions with respect to the Hamiltonian $\tilde{H}_{N+n}$ that specifically uses the independence of the parts that we have constructed. Recall we have to study quantities like those inside the limit on the right hand side of equation (2.6).

Theorem 2.7. Let $f$ be a function of the form $\prod_{l=1}^{n}\left(\hat{\sigma}_{N+l}\right)^{e_{l}}$ from $\mathbb{R}^{n} \rightarrow \mathbb{R}$ where each of the $e_{l}$ is a non-negative integer. We have the following comparison estimate

$$
\begin{aligned}
& \lim _{N \rightarrow \infty}\left|\mathbb{E}\left[\frac{\int_{S_{N+n}^{C, R, n}} f\left(\hat{\sigma}_{N+1}, \ldots, \hat{\sigma}_{N+n}\right) \prod_{i=1}^{n} e^{-\frac{\hat{\sigma}_{N+i}^{2}}{2} e^{H_{N+n}\left(\hat{\sigma}_{1}, \ldots, \hat{\sigma}_{N+n}\right)} \mathrm{d} \hat{\sigma}}}{\int_{S_{N+n}^{C, R, n}} e^{H_{N+n}\left(\hat{\sigma}_{1}, \ldots, \hat{\sigma}_{N+n}\right)} \prod_{i=1}^{n} e^{-\frac{\hat{\sigma}_{N+i}^{2}}{2} \mathrm{~d} \hat{\sigma}}}\right]\right| \leq \mathrm{O}\left(\frac{C^{4+\sum_{l=1}^{n} e_{l}}}{R^{2}}\right), \\
& -\mathbb{E}\left[\frac{\int_{S_{N+n}^{C, R, n}} f\left(\hat{\sigma}_{N+1}, \ldots, \hat{\sigma}_{N+n}\right) \prod_{i=1}^{n} e^{-\frac{\hat{\sigma}_{N+1}^{2}}{2}} e^{\tilde{H}_{N+n}\left(\hat{\sigma}_{1}, \ldots, \hat{\sigma}_{N+n}\right)} \mathrm{d} \hat{\sigma}}{\int_{S_{N+n}^{C, R, n}} e^{\tilde{H}_{N+n}\left(\hat{\sigma}_{1}, \ldots, \hat{\sigma}_{N+n}\right)} \prod_{i=1}^{n} e^{-\frac{\hat{\sigma}_{N+i}^{2}}{2}} \mathrm{~d} \hat{\sigma}}\right] \mid
\end{aligned}
$$

where the constant in the error bound does not depend on $C$ or $R$. In the integrals that have appeared beforehand, $d \hat{\sigma}$ is the product measure $\prod_{i=1}^{N+n} \mathrm{~d} \hat{\sigma}_{i}$.

Proof. For some simplicity of notation, we will denote

$$
\begin{gathered}
\tilde{f}\left(\hat{\sigma}_{N+1}, \ldots, \hat{\sigma}_{N+n}\right):=f\left(\hat{\sigma}_{N+1}, \ldots, \hat{\sigma}_{N+n}\right) \prod_{i=1}^{n} e^{-\frac{\hat{\sigma}_{N+i}^{2}}{2}}, \\
\tilde{g}\left(\hat{\sigma}_{N+1}, \ldots, \hat{\sigma}_{N+n}\right):=\prod_{i=1}^{n} e^{-\frac{\hat{\sigma}_{N+1}^{2}}{2}} .
\end{gathered}
$$

In order to compare the two quantities, we will perform a Gaussian interpolation. We may drop the subscript $N+n$ in some following computations. Let $H_{N+n}, \tilde{H}_{N+n}$ be independent Gaussian processes with the appropriate covariance structure defined earlier, and let

$$
H_{t}\left(\hat{\sigma}_{1}, \ldots, \hat{\sigma}_{N+n}\right):=\sqrt{t} H_{N+n}+\sqrt{1-t} \tilde{H}_{N+n} .
$$

We now consider the quantity

$$
F(t)=\mathbb{E}\left[\frac{\int_{S_{N+n}^{C, R, n}} \tilde{f}\left(\hat{\sigma}_{N+1}, \ldots, \hat{\sigma}_{N+n}\right) e^{H_{t}\left(\hat{\sigma}_{1}, \ldots, \hat{\sigma}_{N+n}\right)} \mathrm{d} \hat{\sigma}}{\int_{S_{N+n}^{C, R, n}} \tilde{g}\left(\hat{\sigma}_{N+1}, \ldots, \hat{\sigma}_{N+n}\right) e^{H_{t}\left(\hat{\sigma}_{1}, \ldots, \hat{\sigma}_{N+n}\right)} \mathrm{d} \hat{\sigma}}\right],
$$

and remark that the quantity (2.28) is $|F(1)-F(0)|$. As is standard, we will derive a bound on the above quantity by bounding the derivative. To simplify notation, we will denote the quantity $Z_{t}:=\int_{S_{N+n}^{C, R, n}} \tilde{g}\left(\hat{\sigma}_{N+1}, \ldots, \hat{\sigma}_{N+n}\right) e^{H_{t}\left(\hat{\sigma}_{1}, \ldots, \hat{\sigma}_{N+n}\right)} \mathrm{d} \hat{\sigma}$. Since we will always integrate over the set $S_{N+n}^{C, R, n}$ we avoid here any specific mention of this set. We see that $F^{\prime}(t)$ is equal to

$$
\begin{aligned}
& \mathbb{E}\left[\frac{1}{2 \sqrt{t}}\left(Z_{t}^{-1} \int \tilde{f}(\hat{\sigma}) H(\hat{\sigma}) e^{H_{t}(\hat{\sigma})} \mathrm{d} \hat{\sigma}-Z_{t}^{-2} \int \tilde{f}(\hat{\sigma}) \tilde{g}(\hat{\rho}) H(\hat{\rho}) e^{H_{t}(\hat{\sigma})+H_{t}(\hat{\rho})} \mathrm{d} \hat{\sigma} \mathrm{d} \hat{\rho}\right)\right. \\
& \left.-\frac{1}{2 \sqrt{1-t}}\left(Z_{t}^{-1} \int \tilde{f}(\hat{\sigma}) \tilde{H}(\hat{\sigma}) e^{H_{t}(\hat{\sigma})} \mathrm{d} \hat{\sigma}-Z_{t}^{-2} \int \tilde{f}(\hat{\sigma}) \tilde{g}(\hat{\rho}) \tilde{H}(\hat{\rho}) e^{H_{t}(\hat{\sigma})+H_{t}(\hat{\rho})} \mathrm{d} \hat{\sigma} \mathrm{d} \hat{\rho}\right)\right]
\end{aligned}
$$




$$
\begin{aligned}
& =\mathbb{E}\left[\int \tilde{f}(\hat{\sigma})[\operatorname{Cov}(H(\hat{\sigma}), H(\hat{\sigma}))-\operatorname{Cov}(\tilde{H}(\hat{\sigma}), \tilde{H}(\hat{\sigma}))] \frac{e^{H_{t}(\hat{\sigma})}}{2 Z_{t}} \mathrm{~d} \hat{\sigma}\right. \\
& -2 \int \tilde{f}(\hat{\sigma}) \tilde{g}(\hat{\rho})[\operatorname{Cov}(H(\hat{\sigma}), H(\hat{\rho}))-\operatorname{Cov}(\tilde{H}(\hat{\sigma}), \tilde{H}(\hat{\rho}))] \frac{e^{H_{t}(\hat{\sigma})+H_{t}(\hat{\rho})}}{2 Z_{t}^{2}} \mathrm{~d} \hat{\sigma} \mathrm{d} \hat{\rho} \\
& -\int \tilde{f}(\hat{\sigma}) \tilde{g}(\hat{\rho})[\operatorname{Cov}(H(\hat{\rho}), H(\hat{\rho}))-\operatorname{Cov}(\tilde{H}(\hat{\rho}), \tilde{H}(\hat{\rho}))] \frac{e^{H_{t}(\hat{\sigma})+H_{t}(\hat{\rho})}}{2 Z_{t}^{2}} \mathrm{~d} \hat{\sigma} \mathrm{d} \hat{\rho} \\
& \left.+\int \tilde{f}(\hat{\sigma}) \tilde{g}(\hat{\rho}) \tilde{g}(\hat{\eta})[\operatorname{Cov}(H(\hat{\rho}), H(\hat{\eta}))-\operatorname{Cov}(\tilde{H}(\hat{\rho}), \tilde{H}(\hat{\eta}))] \frac{e^{H_{t}(\hat{\sigma})+H_{t}(\hat{\rho})+H_{t}(\hat{\eta})}}{Z_{t}^{3}} \mathrm{~d} \hat{\sigma} \mathrm{d} \hat{\rho} \mathrm{d} \hat{\eta}\right] .
\end{aligned}
$$

The first equality merely computed the derivative of the quantities. In order to derive the last expression, we performed an integration by parts.

We bound the quantity on the right hand side of the quality by taking absolute values everywhere. Every appearance of the differences of $|\operatorname{Cov}(H, H)-\operatorname{Cov}(\tilde{H}, \tilde{H})|$ can be bounded by $\frac{C^{4}}{R^{2}}+o(1)$. Applying this bound will decouple the integration variables.

We can thus bound the right hand side of the previous equation in absolute value by

$$
\begin{aligned}
& \left(\frac{C^{4}}{R^{2}}+o(1)\right) \mathbb{E}\left[\frac{1}{2} \frac{\int|\tilde{f}(\hat{\sigma})| e^{H_{t}(\hat{\sigma})} \mathrm{d} \hat{\sigma}}{Z_{t}}+\frac{3}{2} \frac{\int|\tilde{f}(\hat{\sigma})| e^{H_{t}(\hat{\sigma})} \mathrm{d} \hat{\sigma}}{Z_{t}} \frac{\int \tilde{g}(\hat{\rho}) e^{H_{t}(\hat{\rho})} \mathrm{d} \hat{\rho}}{Z_{t}}\right] \\
& +\left(\frac{C^{4}}{R^{2}}+o(1)\right) \mathbb{E}\left[\frac{\int|\tilde{f}(\hat{\sigma})| e^{H_{t}(\hat{\sigma})} \mathrm{d} \hat{\sigma}}{Z_{t}} \frac{\left.\int \tilde{g}(\hat{\rho}) e^{H_{t}(\hat{\rho})} \mathrm{d} \hat{\rho} \int \frac{\tilde{g}(\hat{\eta}) e^{H_{t}(\hat{\eta})} \mathrm{d} \hat{\eta}}{Z_{t}}\right]}{Z_{t}}\right]
\end{aligned}
$$

Notice that we may bound $|\tilde{f}(\hat{\sigma})| \leq C^{\sum_{l=1}^{n} e_{l}}|\tilde{g}(\hat{\sigma})|$ and that $\tilde{g}(\hat{\sigma})$ is a positive quantity. Additionally, $\frac{\int \tilde{g}(\hat{\rho}) e^{H_{t}(\hat{\rho})} \mathrm{d} \hat{\rho}}{Z_{t}}=1$. This suggests that we have $\frac{\int|\tilde{f}(\hat{\sigma})| e^{H_{t}(\hat{\sigma})} \mathrm{d} \hat{\sigma}}{Z_{t}} \leq C^{\sum_{i=1}^{n} e_{l}}$.

Combining the previous inequalities give us the desired result.

The main consequence of this Theorem is the following Corollary, which allows us to express the spin distributions from the Hamiltonian $H_{N}$ in terms of expectations with respect to $\tilde{H}_{N}$.

Corollary 2.8. Let $e_{l, n}$ be non-negative integers. We have the following limiting relations.

$$
\begin{aligned}
& \lim _{N \rightarrow \infty} \mathbb{E}\left[\prod_{l=1}^{k}\left\langle\left(\sigma_{N+1}^{l}\right)^{e_{l, 1}} \ldots\left(\sigma_{N+n}^{l}\right)^{e_{l, n}}\right\rangle_{H_{N+n}\left(S_{N+n}\right)}\right] \\
& =\lim _{C \rightarrow \infty} \lim _{R \rightarrow \infty} \lim _{N \rightarrow \infty} \mathbb{E}\left[\prod_{l=1}^{k} \frac{\int_{S_{N+n}^{C, R, n}} \prod_{i=1}^{n}\left(\hat{\sigma}_{N+i}^{l}\right)^{e_{l, i}} e^{-\frac{\left(\hat{\sigma}_{N+i}^{l}\right)^{2}}{2}} e^{\tilde{H}_{N+n}\left(\hat{\sigma}_{1}^{l}, \ldots, \hat{\sigma}_{N+n}^{l}\right)} \mathrm{d} \hat{\sigma}^{l}}{\int_{S_{N+n}^{C, R, n}} e^{\tilde{H}_{N+n}\left(\hat{\sigma}_{1}^{l}, \ldots, \hat{\sigma}_{N+n}^{l}\right)} \prod_{i=1}^{n} e^{-\frac{\left(\hat{\sigma}_{N+i}^{l}\right)^{2}}{2}} \mathrm{~d} \hat{\sigma}^{l}}\right] .
\end{aligned}
$$

Proof. From Lemma 2.1 and Lemma 2.3, we introduce the $C$ cutoff and introduce the renormalization with respect to $R$. For fixed $R$, we see we have the limiting relation

$$
\begin{aligned}
& \lim _{N \rightarrow \infty} \mathbb{E}\left[\prod_{l=1}^{k}\left\langle\left(\sigma_{N+1}^{l}\right)^{e_{l, 1}} \ldots\left(\sigma_{N+n}^{l}\right)^{e_{l, n}}\right\rangle_{H_{N+n}\left(S_{N+n}\right)}\right] \\
& =\lim _{C \rightarrow \infty} \lim _{N \rightarrow \infty} \mathbb{E}\left[\prod_{l=1}^{k} \frac{\int_{S_{N+n}^{C, R, n}} \prod_{i=1}^{n}\left(\hat{\sigma}_{N+i}^{l}\right)^{e_{l, i}} e^{-\frac{\left(\hat{\sigma}_{N+i}^{l}\right)^{2}}{2}} e^{H_{N+n}\left(\hat{\sigma}_{1}^{l}, \ldots, \hat{\sigma}_{N+n}^{l}\right)} \mathrm{d} \hat{\sigma}^{l}}{\int_{S_{N+n}^{C, R, n}} e^{H_{N+n}\left(\hat{\sigma}_{1}^{l}, \ldots, \hat{\sigma}_{N+n}^{l}\right)} \prod_{i=1}^{n} e^{-\frac{\left(\hat{\sigma}_{N+i}^{l}\right)^{2}}{2}} \mathrm{~d} \hat{\sigma}^{l}}\right] .
\end{aligned}
$$


We remark that $R$ is a free parameter here and

$$
\lim _{N \rightarrow \infty} \mathbb{E}\left[\prod_{l=1}^{k} \frac{\int_{S_{N+n}^{C, R}} \prod_{i=1}^{n}\left(\hat{\sigma}_{N+i}^{l}\right)^{e_{l, i}} e^{-\frac{\left(\hat{\sigma}_{N+i}^{l}\right)^{2}}{2}} e^{H_{N+n}\left(\hat{\sigma}_{1}^{l}, \ldots, \hat{\sigma}_{N+n}^{l}\right)} \mathrm{d} \hat{\sigma}^{l}}{\int_{S_{N+n}^{C, R, n}} e^{H_{N+n}\left(\hat{\sigma}_{1}^{l}, \ldots, \hat{\sigma}_{N+n}^{l}\right)} \prod_{i=1}^{n} e^{-\frac{\left(\hat{\sigma}_{N+i}^{l}\right)^{2}}{2}} \mathrm{~d} \hat{\sigma}^{l}}\right],
$$

is a constant in $R$. We can freely take the limit of $R \rightarrow \infty$. We have,

$$
\begin{aligned}
& \lim _{N \rightarrow \infty} \mathbb{E}\left[\prod_{l=1}^{k}\left\langle\left(\sigma_{N+1}^{l}\right)^{e_{l, 1}} \ldots\left(\sigma_{N+n}^{l}\right)^{e_{l, n}}\right\rangle_{H_{N+n}\left(S_{N+n}\right)}\right] \\
& =\lim _{C \rightarrow \infty} \lim _{R \rightarrow \infty} \lim _{N \rightarrow \infty} \mathbb{E}\left[\prod_{l=1}^{k} \frac{\int_{S_{N+n}^{C, R}, n} \prod_{i=1}^{n}\left(\hat{\sigma}_{N+i}^{l}\right)^{e l, i} e^{-\frac{\left(\hat{\sigma}_{N+i}^{l}\right)^{2}}{2}} e^{H_{N+n}\left(\hat{\sigma}_{1}^{l}, \ldots, \hat{\sigma}_{N+n}^{l}\right)} \mathrm{d} \hat{\sigma}^{l}}{\int_{S_{N+n}^{C, R, n}} e^{H_{N+n}\left(\hat{\sigma}_{1}^{l}, \ldots, \hat{\sigma}_{N+n}^{l}\right)} \prod_{i=1}^{n} e^{-\frac{\left(\hat{\sigma}_{N+i}^{l}\right)^{2}}{2}} \mathrm{~d} \hat{\sigma}^{l}}\right] .
\end{aligned}
$$

Finally, Theorem 2.7 allows us to replace the expectation over Hamiltonian $H_{N+n}$ with Hamiltonian $\tilde{H}_{N+n}$ if we take $\lim _{C \rightarrow \infty} \lim _{R \rightarrow \infty}$ in that order. We derive the equation,

$$
\begin{aligned}
& \lim _{N \rightarrow \infty} \mathbb{E}\left[\prod_{l=1}^{k}\left\langle\left(\sigma_{N+1}^{l}\right)^{e_{l, 1}} \ldots\left(\sigma_{N+n}^{l}\right)^{e_{l, n}}\right\rangle_{H_{N+n}\left(S_{N+n}\right)}\right] \\
& =\lim _{C \rightarrow \infty} \lim _{R \rightarrow \infty} \lim _{N \rightarrow \infty} \mathbb{E}\left[\prod_{l=1}^{k} \frac{\int_{S_{N+n}^{C, R, n}} \prod_{i=1}^{n}\left(\hat{\sigma}_{N+i}^{l}\right)^{e_{l, i}} e^{-\frac{\left(\hat{\sigma}_{N+i}^{l}\right)^{2}}{2}} e^{\tilde{H}_{N+n}\left(\hat{\sigma}_{1}^{l}, \ldots, \hat{\sigma}_{N+n}^{l}\right)} \mathrm{d} \hat{\sigma}^{l}}{\int_{S_{N+n}^{C, R}} e^{\tilde{H}_{N+n}\left(\hat{\sigma}_{1}^{l}, \ldots, \hat{\sigma}_{N+n}^{l}\right)} \prod_{i=1}^{n} e^{-\frac{\left(\hat{\sigma}_{N+i}^{l}\right)^{2}}{2}} \mathrm{~d} \hat{\sigma}^{l}}\right] .
\end{aligned}
$$

This completes the proof.

From this point on, we will now attempt to compute quantities with respect to the distribution using the Hamiltonian $\tilde{H}$; namely, we study quantities such as those appearing on the right hand side of equation (2.30). Applying our cavity decomposition of the Hamiltonian $\tilde{H}$, as detailed in equations (2.13), (2.14) and (2.15), we see that we need to study quantities expressed in the following manner,

$$
\begin{aligned}
& \mathbb{E}\left[\prod_{l=1}^{k} \frac{\int_{S_{N+n}^{C, R, n}} \prod_{i=1}^{n}\left(\hat{\sigma}_{N+i}^{l}\right)^{e l, i} e^{-\frac{\left(\hat{\sigma}_{N+i}^{l}\right)^{2}}{2}} e^{\tilde{H}_{N+n}\left(\hat{\sigma}_{1}^{l}, \ldots, \hat{\sigma}_{N+n}^{l}\right)} \mathrm{d} \hat{\sigma}^{l}}{\int_{S_{N+n}^{C, R, n}} \prod_{i=1}^{n} e^{-\frac{\left(\hat{\sigma}_{N+i}^{l}\right)^{2}}{2}} e^{\tilde{H}_{N+n}\left(\hat{\sigma}_{1}^{l}, \ldots, \hat{\sigma}_{N+n}^{l}\right)} \mathrm{d} \hat{\sigma}^{l}}\right] \\
= & \mathbb{E} \prod_{l=1}^{k} \frac{\left\langle\prod_{i=1}^{n} \int_{[-C, C]} s_{i}^{e, i} e^{-\frac{s_{i}^{2}}{2}+Z^{i}\left(\hat{\sigma}^{l}\right) s_{i}+\left[\sqrt{R^{2}+1}-\frac{s_{i}^{2}}{2 \sqrt{R^{2}+1}}\right] Y^{i}\left(\hat{\sigma}^{l}\right)} \mathrm{d} s_{i}\right\rangle_{\hat{H}_{N}}}{\left\langle\prod_{i=1}^{n} \int_{[-C, C]} e^{-\frac{s_{i}^{2}}{2}+Z^{i}\left(\hat{\sigma}^{l}\right) s_{i}+\left[\sqrt{R^{2}+1}-\frac{s_{i}^{2}}{2 \sqrt{R^{2}+1}}\right] Y^{i}\left(\hat{\sigma}^{l}\right)} \mathrm{d} s_{i}\right\rangle_{\hat{H}_{N}}}
\end{aligned}
$$

\section{Reduction to finite replica symmetry breaking}

We will show that due to ultrametricity,

$$
\lim _{N \rightarrow \infty} \mathbb{E} \prod_{l=1}^{k} \frac{\left\langle\prod_{i=1}^{n} \int_{[-C, C]} s_{i}^{e l, i} e^{-\frac{s_{i}^{2}}{2}+Z^{i}\left(\hat{\sigma}^{l}\right) s_{i}+\left[\sqrt{R^{2}+1}-\frac{s_{i}^{2}}{2 \sqrt{R^{2}+1}}\right] Y^{i}\left(\hat{\sigma}^{l}\right)} \mathrm{d} s_{i}\right\rangle_{\hat{H}_{N}\left(S_{N-R^{2}}\right)}}{\left\langle\prod_{i=1}^{n} \int_{[-C, C]} e^{-\frac{s_{i}^{2}}{2}+Z^{i}\left(\hat{\sigma}^{l}\right) s_{i}+\left[\sqrt{R^{2}+1}-\frac{s_{i}^{2}}{2 \sqrt{R^{2}+1}}\right] Y^{i}\left(\hat{\sigma}^{l}\right)} \mathrm{d} s_{i}\right\rangle_{\hat{H}_{N}\left(S_{N-R^{2}}\right)}},
$$

only depends on the Hamiltonian $\hat{H}_{N}$ through its limiting overlap distribution $\zeta^{*}$ (Recall that the notation $\zeta^{*}$ first appeared in Theorem 1.2). The expression inside the limit 
of (3.1) can be understood as a continuous function $F^{R, C, E}(\zeta)$ of $\zeta$ in the space of probability measures on $[0,1]$ with the weak ${ }^{*}$ topology, where $E$ is the set of all values $e_{l, i}$, evaluated at $\zeta^{*}$. (As before, we restrict the values of $e_{l, i}$ to be non-negative integers.)

Using the results of Corollary 2.8, we are able to derive the fact that

$$
\lim _{N \rightarrow \infty} \mathbb{E} \prod_{l=1}^{k}\left\langle\left(\sigma_{1}^{l}\right)^{e_{l, 1}} \ldots\left(\sigma_{n}^{l}\right)^{e_{l, n}}\right\rangle_{H_{N}\left(S_{N}\right)}=\lim _{C \rightarrow \infty} \lim _{R \rightarrow \infty} F^{R, C, E}\left(\zeta^{*}\right) .
$$

What we would like to do is to reduce the computation to when $\zeta^{*}$ satisfies finite replica symmetry breaking. Let $\zeta_{i}$ be a sequence of probability measures approaching $\zeta^{*}$ in the weak* topology.

We have that

$$
\lim _{N \rightarrow \infty} \mathbb{E} \prod_{l=1}^{k}\left\langle\left(\sigma_{1}^{l}\right)^{e_{l, 1}} \ldots\left(\sigma_{n}^{l}\right)^{e_{l, n}}\right\rangle=\lim _{C \rightarrow \infty} \lim _{R \rightarrow \infty} \lim _{i \rightarrow \infty} F^{R, C, E}\left(\zeta_{i}\right) .
$$

We would like to exchange the limits so that we can write the limit as

$$
\lim _{i \rightarrow \infty} \lim _{C \rightarrow \infty} \lim _{R \rightarrow \infty} F^{R, C, E}\left(\zeta_{i}\right) .
$$

This would involve showing the uniform approach of $F^{R, C, E}\left(\zeta_{i}\right)$ to its limit in $\zeta$. We will proceed to justify this exchange of limits in the following sections.

\subsection{Computation of $F^{R, C, E}(\zeta)$ under finite replica symmetry breaking}

Lemma 3.1. There is some function $F^{R, C, E}(\zeta)$ continuous on the weak ${ }^{*}$ topology of probability measures on $[0,1]$ with

$$
\lim _{N \rightarrow \infty} \mathbb{E}\left[\prod_{l=1}^{k} \frac{\int_{S_{N+n}^{C, R, n}} \prod_{j=1}^{n}\left(\hat{\sigma}_{N+j}^{l}\right)^{e_{l, j}} e^{-\frac{\left(\hat{\sigma}_{N+j}^{l}\right)^{2}}{2}} e^{\tilde{H}_{N+n}\left(\hat{\sigma}^{l}\right)} \mathrm{d} \hat{\sigma}^{l}}{\int_{S_{N+n}^{C, R}, n} \prod_{j=1}^{n} e^{-\frac{\left(\hat{\sigma}_{N+j}^{l}\right)^{2}}{2}} e^{\tilde{H}_{N+n}\left(\hat{\sigma}^{l}\right)} \mathrm{d} \hat{\sigma}^{l}}\right]=F^{R, C, E}\left(\zeta^{*}\right),
$$

and for measures $\zeta$ satisfying finite replica symmetry breaking with support at points $0=q_{0} \leq q_{1} \leq \ldots \leq q_{r}=q_{*}$, we have

$$
F^{C, R, E}(\zeta)=\mathbb{E} \prod_{l=1}^{k} \frac{\sum_{\alpha_{l}} w_{\alpha_{l}} \prod_{i=1}^{n} \int_{-C}^{C} s_{i}^{e_{l, i}} e^{-\frac{1}{2}\left[1+\hat{b}_{i n}\right] s_{i}^{2}+M_{i}\left(h_{\alpha_{l}}\right)} \mathrm{d} s_{i}}{\sum_{\alpha_{l}} w_{\alpha_{l}} \prod_{i=1}^{n} \int_{-C}^{C} e^{-\frac{1}{2}\left[1+\hat{b}_{i n}\right] s_{i}^{2}+M_{i}\left(h_{\alpha_{l}}\right)} \mathrm{d} s_{i}},
$$

where the quantities on the right hand side of the above expression are computed with respect to an RPC [12][Ch 2.3] whose overlap distribution is given by $\zeta . \mathfrak{Y}_{i}$ and $\mathfrak{Z}_{i}$ are independent Gaussian processes with covariance given by

$$
\begin{aligned}
& \operatorname{Cov}\left(\mathfrak{Y}_{i}\left(h_{\alpha}\right), \mathfrak{Y}_{i}\left(h_{\beta}\right)\right)=\left\langle h_{\alpha}, h_{\beta}\right\rangle \xi^{\prime}\left(\left\langle h_{\alpha}, h_{\beta}\right\rangle\right), \\
& \operatorname{Cov}\left(\mathfrak{Z}_{i}\left(h_{\alpha}\right), \mathfrak{Z}_{i}\left(h_{\beta}\right)\right)=\xi^{\prime}\left(\left\langle h_{\alpha}, h_{\beta}\right\rangle\right),
\end{aligned}
$$

where $l$ is a replica index for $\alpha_{l}, e_{l, i}$ is an exponent, and $\left.\langle\cdot, \cdot\rangle\right\rangle$ is the inner product.

In the expression (3.5), we use $\hat{b}_{\text {in }}:=\xi^{\prime}\left(q_{*}\right)\left(1-q_{*}\right)$ and

$$
M_{i}\left(h_{\alpha}\right)=\mathfrak{Z}_{i}\left(h_{\alpha_{l}}\right) s_{i}+\mathfrak{Y}_{i}\left(h_{\alpha_{l}}\right)\left[\sqrt{R^{2}+1}-\frac{s_{i}^{2}}{2 \sqrt{R^{2}+1}}\right]+\frac{\left(\xi^{\prime}(1)-q_{*} \xi^{\prime}\left(q_{*}\right)\right) s_{i}^{4}}{4\left(R^{2}+1\right)} .
$$

We will use this shorthand when useful and replace it with more explicit representations when it is more germane to the proof. 
Proof. Part 1: The weak continuity of $F^{R, C, E}$ as a function of $\zeta$

For simplicity, we will write out the proof in the one replica case and a one particle cavity $e_{1,1}=1$. We will show the expression inside the limit of (3.1) can be shown to be a bounded continuous function of the overlap distribution. The logic will broadly follow that of [12][Thm 1.3], this time applying the approximation and cutoff procedures to the function $\frac{1}{x}$.

For some convenience of notation, we use the notation

$$
\begin{aligned}
& \mathcal{E}_{N}:=\left\langle\int_{[-C, C]} e^{-\frac{s^{2}}{2}+Z^{1}(\hat{\sigma}) s+\left[\sqrt{R^{2}+1}-\frac{s^{2}}{2 \sqrt{R^{2}+1}}\right] Y^{1}(\hat{\sigma})} \mathrm{d} s\right\rangle_{\hat{H}_{N}(\hat{\sigma})}, \\
& \mathcal{F}_{N}:=\left\langle\int_{[-C, C]} s e^{-\frac{s^{2}}{2}+Z^{1}(\hat{\sigma}) s+\left[\sqrt{R^{2}+1}-\frac{s^{2}}{2 \sqrt{R^{2}+1}}\right] Y^{1}(\hat{\sigma})} \mathrm{d} s\right\rangle_{\hat{H}_{N}(\hat{\sigma})} .
\end{aligned}
$$

First, fix some small $\epsilon>0$. We let $F_{\epsilon}$ be the function defined by

$$
F_{\epsilon}(x):=\epsilon \mathbb{1}(x \leq \epsilon)+x \mathbb{1}\left(\epsilon<x<\epsilon^{-1}\right)+\epsilon^{-1} \mathbb{1}\left(x \geq \epsilon^{-1}\right),
$$

and $G_{\epsilon}$ be defined as

$$
G_{\epsilon}(x):=\operatorname{sign}(x) C \epsilon^{-1} \mathbb{1}\left(|x| \geq C \epsilon^{-1}\right)+x \mathbb{1}\left(|x|<C \epsilon^{-1}\right) .
$$

These functions are introduced to regularize the numerator and denominator.

We see that since $\left|\mathcal{F}_{N}\right| \leq C \mathcal{E}_{N}$, we also have the relation $\left|G_{\epsilon}\left(\mathcal{F}_{N}\right)\right| \leq C F_{\epsilon}\left(\mathcal{E}_{N}\right)$. We first establish,

$$
\mathbb{E}\left|\frac{\mathcal{F}_{N}}{\mathcal{E}_{N}}-\frac{G_{\epsilon}\left(\mathcal{F}_{N}\right)}{F_{\epsilon}\left(\mathcal{E}_{N}\right)}\right| \leq 2 C \mathbb{P}\left(\epsilon \geq \mathcal{E}_{N}, \mathcal{E}_{N} \geq \epsilon^{-1}\right) .
$$

The results of Lemma 3.2 show that the probability in the previous line is bounded by a constant $K$ times $\epsilon$.

Now, we regularize the quantities $\mathcal{E}_{N}$ and $\mathcal{F}_{N}$ as follows. We define,

$$
\begin{aligned}
& \mathcal{E}_{N}^{\mathrm{reg}}:=\left\langle\int_{[-C, C]} e^{-\frac{s^{2}}{2}+G_{-k \log \epsilon}\left(Z^{1}(\hat{\sigma})\right) s+\left[\sqrt{R^{2}+1}-\frac{s^{2}}{2 \sqrt{R^{2}+1}}\right] G_{-k \log \epsilon}\left(Y^{1}(\hat{\sigma})\right)} \mathrm{d} s\right\rangle_{\hat{H}_{N}(\hat{\sigma})} \\
& \mathcal{F}_{N}^{\mathrm{reg}}:=\left\langle\int_{[-C, C]} s e^{\left.-\frac{s^{2}}{2}+G_{-k \log \epsilon}\left(Z^{1}(\hat{\sigma})\right) s+\left[\sqrt{R^{2}+1}-\frac{s^{2}}{2 \sqrt{R^{2}+1}}\right] G_{-k \log \epsilon\left(Y^{1}(\hat{\sigma})\right)} \mathrm{d} s\right\rangle_{\hat{H}_{N}(\hat{\sigma})} .} .\right.
\end{aligned}
$$

The constant $k$ is chosen small enough so that $F_{\epsilon}\left(\mathcal{E}_{N}^{\text {reg }}\right)=\mathcal{E}_{N}^{\text {reg }}$ and $G_{\epsilon}\left(\mathcal{F}_{N}^{\text {reg }}\right)=\mathcal{F}_{N}^{\text {reg }}$ for all $\epsilon$.

Let $\chi$ be the indicator function for the event

$$
\left\{\left\langle\int_{[-C, C]} e^{-s^{2}+2 Z^{1}(\hat{\sigma}) s+2\left[\sqrt{R^{2}+1}-\frac{s^{2}}{2 \sqrt{R^{2}+1}}\right] Y^{1}(\hat{\sigma})} \mathrm{d} s\right\rangle_{\hat{H}_{N}(\hat{\sigma})} \leq \epsilon^{-1}\right\} \cap\left\{\epsilon \leq \mathcal{E}_{N} \leq \epsilon^{-1}\right\} .
$$

We see that we now have,

$$
\begin{aligned}
\mathbb{E}\left|\frac{G_{\epsilon}\left(\mathcal{F}_{N}\right)}{F_{\epsilon}\left(\mathcal{E}_{N}\right)}-\frac{G_{\epsilon}\left(\mathcal{F}_{N}^{\mathrm{reg}}\right)}{F_{\epsilon}\left(\mathcal{E}_{N}^{\mathrm{reg}}\right)}\right| & \leq \mathbb{E}\left[\left|\frac{\mathcal{F}_{\mathcal{N}}}{\mathcal{E}_{N}}-\frac{\mathcal{F}_{N}^{\mathrm{reg}}}{\mathcal{E}_{N}^{\mathrm{reg}}}\right| \chi\right]+2 C \mathbb{E}(1-\chi) \\
& \leq \mathbb{E}\left|\frac{\mathcal{F}_{N}\left(\mathcal{E}_{N}^{\mathrm{reg}}-\mathcal{E}_{N}\right)}{\mathcal{E}_{N} \mathcal{E}_{N}^{\mathrm{reg}}}\right| \chi+\mathbb{E}\left|\frac{\left(\mathcal{F}_{N}^{\mathrm{reg}}-\mathcal{F}_{N}\right)}{\mathcal{E}_{N}^{\mathrm{reg}}}\right| \chi+2 C \mathbb{E}(1-\chi) \\
& \leq C \epsilon^{-1} \mathbb{E}\left|\mathcal{E}_{N}-\mathcal{E}_{N}^{\mathrm{reg}}\right| \chi+\epsilon^{-1} \mathbb{E}\left|\mathcal{F}_{N}-\mathcal{F}_{N}^{\mathrm{reg}}\right| \chi+2 C \mathbb{E}(1-\chi)
\end{aligned}
$$


To get from the second to the third line, we used the following facts: $\frac{\mathcal{F}_{N}}{\mathcal{E}_{N}} \leq C$ and $\mathcal{E}_{N}^{\text {reg }} \geq \epsilon$. We remark again that the estimates of Lemma 3.2 along with a union bound control $\mathbb{E}(1-\chi)$.

We now control the difference $\mathbb{E}\left|\mathcal{E}_{N}-\mathcal{E}_{N}^{\text {reg }}\right| \chi$. Controlling the other term is similar. We have, by using the definition of $\mathcal{E}_{N}$ and $\mathcal{E}_{N}^{\text {reg }}$,

$$
\begin{aligned}
& \mathbb{E} \mid \mathcal{E}_{N}- \mathcal{E}_{N}^{\mathrm{reg}} \mid \chi \\
& \leq \mathbb{E}\left\langle\mathbb{1}\left(\left|Z^{1}(\hat{\sigma})\right| \text { or }\left|Y^{1}(\hat{\sigma})\right| \geq-k \log \epsilon\right) \int_{-C}^{C} e^{-s^{2} / 2+Z^{1}(\hat{\sigma}) s+\left[\sqrt{R^{2}+1}-\frac{s^{2}}{2 \sqrt{R^{2}+1}}\right] Y^{1}(\hat{\sigma})} \mathrm{d} s\right\rangle \chi \\
&+\mathbb{E}\left\langle\mathbb{1}\left(\left|Z^{1}(\hat{\sigma})\right| \text { or }\left|Y^{1}(\hat{\sigma})\right| \geq-k \log \epsilon\right)\right. \\
&\left.\quad \times \int_{-C}^{C} e^{-s^{2} / 2+G_{-k \log \epsilon}\left(Z^{1}(\hat{\sigma})\right) s+\left[\sqrt{R^{2}+1}-\frac{s^{2}}{2 \sqrt{R^{2}+1}}\right] G_{-k \log \epsilon}\left(Y^{1}(\hat{\sigma})\right)} \mathrm{d} s\right\rangle \chi .
\end{aligned}
$$

We now bound the quantity in the second line of the above expression. Bounding the other quantity is similar,

$$
\begin{aligned}
& \mathbb{E}\left\langle\mathbb{1}\left(\left|Z^{1}(\hat{\sigma})\right| \text { or }\left|Y^{1}(\hat{\sigma})\right| \geq-k \log \epsilon\right) \int_{-C}^{C} e^{-s^{2} / 2+Z^{1}(\hat{\sigma}) s+\left[\sqrt{R^{2}+1}-\frac{s^{2}}{2 \sqrt{R^{2}+1}}\right] Y^{1}(\hat{\sigma})} \mathrm{d} s\right\rangle \chi \\
& \leq \mathbb{E}\left\langle\mathbb{1}\left(\left|Z^{1}(\hat{\sigma})\right| \text { or }\left|Y^{1}(\hat{\sigma})\right| \geq-k \log \epsilon\right)\right\rangle^{1 / 2} \\
& \quad \times\left\langle\left(\int_{-C}^{C} e^{-s^{2} / 2+Z^{1}(\hat{\sigma}) s+\left[\sqrt{R^{2}+1}-\frac{s^{2}}{2 \sqrt{R^{2}+1}}\right] Y^{1}(\hat{\sigma})} \mathrm{d} s\right)^{2}\right\rangle^{1 / 2} \chi \\
& \leq\left(\mathbb{E}\left\langle\mathbb{1}\left(\left|Z^{1}(\hat{\sigma})\right| \text { or }\left|Y^{1}(\hat{\sigma})\right| \geq-k \log \epsilon\right)\right\rangle\right)^{1 / 2} \\
& \quad \times\left(\mathbb{E}\left\langle\left(\int_{-C}^{C} e^{-s^{2} / 2+Z^{1}(\hat{\sigma}) s+\left[\sqrt{R^{2}+1}-\frac{s^{2}}{2 \sqrt{R^{2}+1}}\right] Y^{1}(\hat{\sigma})} \mathrm{d} s\right)^{2}\right\rangle\right)^{1 / 2} \\
& \leq\left(\mathbb{E}\left\langle\mathbb{1}\left(\left|Z^{1}(\hat{\sigma})\right| \text { or }\left|Y^{1}(\hat{\sigma})\right| \geq-k \log \epsilon\right)\right\rangle\right)^{1 / 2} \\
& \quad \times\left(2 C \mathbb{E}\left\langle\int_{-C}^{C} e^{-s^{2}+2 Z^{1}(\hat{\sigma}) s+2\left[\sqrt{R^{2}+1}-\frac{s^{2}}{2 \sqrt{R^{2}+1}}\right] Y^{1}(\hat{\sigma})} \mathrm{d} s\right\rangle \chi\right)^{1 / 2} \\
& \leq(2 C)^{1 / 2} \epsilon^{-1 / 2}\left(\mathbb{E}\left\langle\mathbb{1}\left(\left|Z^{1}(\hat{\sigma})\right| \text { or }\left|Y^{1}(\hat{\sigma})\right| \geq-k \log \epsilon\right)\right\rangle\right)^{1 / 2} .
\end{aligned}
$$

We used the Cauchy-Schwartz inequality to derive the first inequality (in \langle\rangle ), the second inequality (in $\mathbb{E}$ ), and the third inequality (in the integral $\int_{-C}^{C}$ ). To get the final inequality, we used the definition of $\chi$.

Since $Z^{1}(\hat{\sigma})$ and $Y^{1}(\hat{\sigma})$ are Gaussians, we have that

$$
\left(\mathbb{E}\left\langle\mathbb{1}\left(\left|Z^{1}(\hat{\sigma})\right| \text { or }\left|Y^{1}(\hat{\sigma})\right| \geq-k \log \epsilon\right)\right\rangle\right)^{1 / 2} \leq e^{-K(\log \epsilon)^{2}},
$$

for some constant $K$. As $\epsilon$ goes to 0 , we find that $\epsilon^{-3 / 2} e^{-K(\log \epsilon)^{2}}=e^{-3 / 2 \log \epsilon-K(\log \epsilon)^{2}}$ goes to 0 .

This argument shows that up to an error that vanishes in $\epsilon$, we have $\mathbb{E} \frac{\mathcal{F}_{N}}{\mathcal{E}_{N}}=\mathbb{E} \frac{\mathcal{F}_{\mathcal{E}}^{\text {reg }}}{\mathcal{E}_{N}^{\text {reg }}}$. The function $\frac{1}{x}$ can be approximated uniformly by a polynomial on the domain $\left[\epsilon, \epsilon^{-1}\right]$. This says that to an arbitrarily small error, we can find a polynomial, $P$, such that $\frac{\mathbb{E}^{N}}{\mathcal{\mathcal { E }}_{N}^{\text {reg }}}=$ $\mathbb{E} \mathcal{F}_{N}^{\text {reg }} P\left(\mathcal{E}_{N}^{\text {reg }}\right)$. By expanding the polynomial, we obtain replicas of the $\hat{\sigma}$. Integrating first over the randomness of the Gaussians $Z^{1}$ and $Y^{1}$ will show that $\mathbb{E} \mathcal{F}_{N}^{\text {reg }} P\left(\mathcal{E}_{N}^{\text {reg }}\right)$ is some weakly continuous function of the law of a finite component of the infinite replica 
overlap matrix. We now see that the function $F^{C, R, E}$ is arbitrarily close to a function that is weakly continuous in the law of infinite replica overlap matrix and will thus be weakly continuous itself.

Panchenko's ultrametricity theorem [12][Thm 2.14] says that the law of the infinite overlap matrix is a continuous function of the law of the limiting overlap distribution. This shows that $F^{C, R, E}$ is weakly continuous in the law of the limiting overlap distribution of $\hat{H}_{N}$.

We finally have to show that the limiting overlap distribution of $\hat{H}_{N}$ would be the same as the limiting overlap distribution of $H_{N}$. Observe that both $\hat{H}_{N}$ and $H_{N}$ are both generic spin-glasses. [12][Thm 3.7] says that if these models have the same limiting free energy, then they would have the same limiting overlap distributions.

Showing that these two spin glasses have the same limiting free energy is an exercise in interpolation. The covariance structure of $\hat{H}_{N}$ is given by $\operatorname{Cov}\left(\hat{H}_{N}(\hat{\sigma}), \hat{H}_{N}(\hat{\rho})\right)=$ $(N+n) \sum_{p=2}^{\infty} \beta_{p}^{2}\left(\frac{1}{N+n} \sum_{i=1}^{N} \hat{\sigma}_{i} \hat{\rho}_{i}\right)^{p}$, while the covariance structure of $H_{N}$ is given by $\operatorname{Cov}\left(H_{N}(\hat{\sigma}), H_{N}(\hat{\rho})\right)=N \sum_{p=2}^{\infty} \beta_{p}^{2}\left(\frac{1}{N} \sum_{i=1}^{N} \hat{\sigma}_{i} \hat{\rho}_{i}\right)^{p}$.

Calculations similar to those appearing in Theorem 2.7 will show that as long as $\lim _{N \rightarrow \infty} \frac{1}{N}\left[\operatorname{Cov}\left(\hat{H}_{N}(\hat{\sigma}), \hat{H}_{N}(\hat{\rho})\right)-\operatorname{Cov}\left(H_{N}(\hat{\sigma}), H_{N}(\hat{\rho})\right)\right]=0$, then the limiting free energies will be the same. The two covariance structures considered satisfy this condition. This completes the proof.

Step 2: The Finite Replica Symmetry Breaking Formula

We have already shown that the function $F^{C, R, E}$ is a weakly continuous function of the overlap distribution of $\hat{H}_{N}$. When this overlap distribution satisfies finite symmetry breaking, it can be approximated by that of a Ruelle Probability Cascade.

Assume the overlap distribution has support given by

$$
0=q_{0} \leq q_{1} \leq q_{2} \leq \ldots \leq q_{r}=q_{*}<1 .
$$

Denote the leaves of the Ruelle probability cascade by $h_{\alpha}$.

We now have to adapt the Gaussian processes $Z^{1}$ and $Y^{1}$ to this RPC structure. As one might expect, we would have the covariance structure

$$
\begin{aligned}
& \operatorname{Cov}\left(\mathfrak{Y}_{1}\left(h_{\alpha}\right), \mathfrak{Y}_{1}\left(h_{\beta}\right)\right)=\left\langle h_{\alpha}, h_{\beta}\right\rangle \xi^{\prime}\left(\left\langle h_{\alpha}, h_{\beta}\right\rangle\right), \\
& \operatorname{Cov}\left(\mathfrak{Z}_{1}\left(h_{\alpha}\right), \mathfrak{Z}_{1}\left(h_{\beta}\right)\right)=\xi^{\prime}\left(\left\langle h_{\alpha}, h_{\beta}\right\rangle\right) .
\end{aligned}
$$

This is analogous to the covariance structure

$$
\begin{aligned}
& \operatorname{Cov}\left(Y^{1}(\hat{\sigma}), Y^{1}(\hat{\rho})\right)=R_{\hat{\sigma}, \hat{\rho}} \xi^{\prime}\left(R_{\hat{\sigma}, \hat{\rho}}\right), \\
& \operatorname{Cov}\left(Z^{1}(\hat{\sigma}), Z^{1}(\hat{\rho})=\xi^{\prime}\left(R_{\hat{\sigma}, \hat{\rho}}\right),\right.
\end{aligned}
$$

at all overlaps except when $\hat{\sigma}=\hat{\rho}$. (Recall that $R_{\hat{\sigma}, \hat{\rho}}$ is just an inner product in $\mathbb{R}^{N}$.)

To match the self-covariance, we see that the quantity corresponding to (3.1) in the RPC would be written as,

$$
\mathbb{E}\left[\frac{\sum_{\alpha} w_{\alpha} \mathbb{E}_{\tilde{\mathfrak{Z}}, \tilde{\mathfrak{Y}}} \int_{[-C, C]} s e^{-\frac{s^{2}}{2}+\left(\mathfrak{Z}_{1}\left(h_{\alpha}\right)+\tilde{\mathfrak{Z}}\right) s+\left(\mathfrak{Y}_{1}\left(h_{\alpha}\right)+\tilde{\mathfrak{Y}}\right)\left[\sqrt{R^{2}+1}-\frac{s^{2}}{2 \sqrt{R^{2}+1}}\right]} \mathrm{d} s}{\sum_{\alpha} w_{\alpha} \mathbb{E}_{\tilde{\mathfrak{Z}}, \tilde{\mathfrak{Y}}} \int_{[-C, C]} e^{-\frac{s^{2}}{2}+\left(\mathfrak{Z}_{1}\left(h_{\alpha}\right)+\tilde{\mathfrak{Z}}\right) s+\left(\mathfrak{Y}_{1}\left(h_{\alpha}\right)+\tilde{\mathfrak{Y}}\right)\left[\sqrt{R^{2}+1}-\frac{s^{2}}{2 \sqrt{R^{2}+1}}\right]} \mathrm{d} s}\right],
$$

where $\tilde{\mathfrak{Z}}$ and $\tilde{\mathfrak{Y}}$ are independent Gaussians with variances $\xi^{\prime}(1)-\xi^{\prime}\left(q_{*}\right)$ and $\xi^{\prime}(1)-q_{*} \xi^{\prime}\left(q_{*}\right)$, respectively. Intuitively speaking, the introduction of these expectations ensures that we have the correct covariance structure at the diagonal. A more formal proof to show that this is the correct expression follows the strategy of approximating the denominator by a polynomial as in Part 1 of this proof. We informally illustrate the gist of the computation. 
Denote the denominator by $\mathcal{E}$ and let $P$ be the polynomial such that $\left|P(x)-x^{-1}\right| \leq \delta$ on $\left[\epsilon, \epsilon^{-1}\right]$ as in the previous part. Roughly speaking, the ratio in (3.18) would be similar to

$$
\mathbb{E}\left[P(\mathcal{E}) \sum_{\alpha} w_{\alpha} \mathbb{E}_{\tilde{\mathfrak{Z}}, \tilde{\mathfrak{Y}}} \int_{[-C, C]} s e^{-\frac{s^{2}}{2}+\left(\mathfrak{Z}_{1}\left(h_{\alpha}\right)+\tilde{\mathfrak{Z}}\right) s+\left(\mathfrak{Y}_{1}\left(h_{\alpha}\right)+\tilde{\mathfrak{Y}}\right)\left[\sqrt{R^{2}+1}-\frac{s^{2}}{2 \sqrt{R^{2}+1}}\right]} \mathrm{d} s\right] .
$$

The polynomial can be expanded, so it looks like we are considering an expectation of a multi-replica quantity. We evaluate this multi-replica expectation by first taking the expectation of all the $\mathfrak{Y}$ and $\mathfrak{Z}$ Gaussians and afterwards taking the expectation over the Dirichlet-Process variables $w_{\alpha}$. For example, if the polynomial $P(x)=\sum_{k} a_{k} x^{k}$, we see that we can write the expression (3.19) as

$$
\begin{aligned}
\mathbb{E} \sum_{k} & a_{k} \sum_{\beta, \alpha_{1}, \ldots, \alpha_{k}} w_{\beta} w_{\alpha_{1}} \ldots w_{\alpha_{k}} \\
& \mathbb{E}_{\mathfrak{Z}, \tilde{\mathfrak{Z}}, \mathfrak{Y}, \tilde{\mathfrak{Y}})} \mathcal{N}\left(\mathfrak{Z}_{1}\left(h_{\beta}\right)+\tilde{\mathfrak{Z}}_{\beta}, \mathfrak{Y}_{1}\left(h_{\beta}\right)+\tilde{\mathfrak{Y}}_{\beta}\right) \prod_{i=1}^{k} D\left(\mathfrak{Z}_{1}\left(h_{\alpha_{k}}\right)+\tilde{\mathfrak{Z}}_{\alpha_{k}}, \mathfrak{Y}_{1}\left(h_{\alpha_{k}}\right)+\tilde{\mathfrak{Y}}_{\alpha_{k}}\right) .
\end{aligned}
$$

where $\mathcal{N}$ is a shorthand for the function $\left.\int_{[-C, C]} s e^{-\frac{s^{2}}{2}+(\mathfrak{Z}+\tilde{\mathfrak{Z}}) s+(\mathfrak{Y}+\tilde{\mathfrak{Y}})\left[\sqrt{R^{2}+1}-\frac{s^{2}}{2 \sqrt{R^{2}+1}}\right]}\right]_{\mathrm{d} s}^{(3.20)}$ as a function of the random variables $\mathfrak{Z}, \mathfrak{Y}, \tilde{\mathfrak{Z}}, \tilde{\mathfrak{Y}}$. $D$ will be a shorthand for the function $\int_{[-C, C]} e^{-\frac{s^{2}}{2}+(\tilde{\mathfrak{Z}}+\tilde{\mathfrak{Z}}) s+(\mathfrak{Y}+\tilde{\mathfrak{Y}})\left[\sqrt{R^{2}+1}-\frac{s^{2}}{2 \sqrt{R^{2}+1}}\right]} \mathrm{d} s . \tilde{\mathfrak{Z}}_{\alpha_{k}}, \tilde{\mathfrak{Y}}_{\alpha_{k}}$ are independent copies of $\tilde{\mathfrak{Z}}$ and $\tilde{\mathfrak{Y}}$ associated with each new node $\alpha_{k}, \beta$.

The expectation,

$$
\mathbb{E}_{\mathfrak{Z}, \tilde{\mathfrak{Z}}, \mathfrak{Y}, \tilde{\mathfrak{Y}}} \mathcal{N}\left(\mathfrak{Z}_{1}\left(h_{\beta}\right), \tilde{\mathfrak{Z}}_{\beta}, \mathfrak{Y}_{1}\left(h_{\beta}\right), \tilde{\mathfrak{Y}}_{\beta}\right) \prod_{i=1}^{k} D\left(\mathfrak{Z}_{1}\left(h_{\alpha_{k}}\right), \tilde{\mathfrak{Z}}_{\alpha_{k}}, \mathfrak{Y}_{1}\left(h_{\alpha_{k}}\right), \tilde{\mathfrak{Y}}_{\alpha_{k}}\right),
$$

will be some function of the covariance matrix of the Gaussian random variables $\left\{\mathfrak{Z}_{1}\left(h_{\alpha_{k}}\right)+\tilde{\mathfrak{Z}}_{\alpha_{k}}\right\} \cup\left\{\mathfrak{Y}_{1}\left(h_{\alpha_{k}}\right)+\tilde{\mathfrak{Y}}_{\alpha_{k}}\right\}$. The introduction of the variables $\tilde{\mathfrak{Y}}$ and $\tilde{\mathfrak{Z}}$ ensures this covariance matrix will be correct along the diagonal.

We obtain our final expresson by integrating over the variables $\tilde{\mathfrak{Z}}$ and $\tilde{\mathfrak{Y}}$.

$$
\begin{aligned}
& \mathbb{E}\left[\frac{\sum_{\alpha} w_{\alpha} \mathbb{E}_{\tilde{\mathfrak{Z}}, \tilde{\mathfrak{Y}}} \int_{[-C, C]} s e^{-\frac{s^{2}}{2}+\left(\mathfrak{Z}_{1}\left(h_{\alpha}\right)+\tilde{\mathfrak{Z}}\right) s+\left(\mathfrak{Y}_{1}\left(h_{\alpha}\right)+\tilde{\mathfrak{Y}}\right)\left[\sqrt{R^{2}+1}-\frac{s^{2}}{2 \sqrt{R^{2}+1}}\right]} \mathrm{d} s}{\sum_{\alpha} w_{\alpha} \mathbb{E}_{\tilde{\mathfrak{Z}}, \tilde{\mathfrak{Y}}} \int_{[-C, C]} e^{-\frac{s^{2}}{2}+\left(\mathfrak{Z}_{1}\left(h_{\alpha}\right)+\tilde{\mathfrak{Z}}\right) s+\left(\mathfrak{Y}_{1}\left(h_{\alpha}\right)+\tilde{\mathfrak{Y}}\right)\left[\sqrt{R^{2}+1}-\frac{s^{2}}{2 \sqrt{R^{2}+1}}\right]} \mathrm{d} s}\right] \\
& =\mathbb{E}\left[\frac{\sum_{\alpha} w_{\alpha} \int_{[-C, C]} s e^{-\frac{s^{2}}{2}\left[1+\hat{b}_{\text {in }}\right]+M_{i}\left(h_{\alpha}\right)} \mathrm{d} s}{\sum_{\alpha} w_{\alpha} \int_{[-C, C]} e^{-\frac{s^{2}}{2}\left[1+\hat{b}_{\text {in }}\right]+M_{i}\left(h_{\alpha}\right)} \mathrm{d} s}\right] .
\end{aligned}
$$

This is the desired result.

We have the following lemma to bound the denominator. We introduce the notation $\mathrm{d} v_{N}^{R}$ to be the Gibbs measure associated with $\hat{H}_{N}$.

Lemma 3.2. For any $k$, we have the following estimate.

$$
P\left(\int_{S_{N}\left(N-R^{2}\right)} \int_{-C}^{C} e^{-\frac{s^{2}}{2}+Z^{1}(\hat{\sigma}) s+\left[\sqrt{R^{2}+1}-\frac{s^{2}}{2 \sqrt{R^{2}+1}}\right] Y^{1}(\hat{\sigma})} \mathrm{d} s \mathrm{~d} \nu_{N}^{R} \leq \frac{1}{L}\right) \leq \frac{K(k)}{L^{k}},
$$


for some constant $K(k)$ that does not depend on $L$. We introduce the parameter $k$ to show that the left hand side of the above inequality decays faster than any polynomial power.

Proof. We have the following

$$
\begin{aligned}
& \int_{-C}^{C} \exp \left\{-\frac{s^{2}}{2}+s Z^{1}(\hat{\sigma})+\left[\sqrt{R^{2}+1}-\frac{s^{2}}{2 \sqrt{R^{2}+1}}\right] Y^{1}(\hat{\sigma})\right\} \mathrm{d} s \\
& =\int_{0}^{C} \exp \left\{-\frac{s^{2}}{2}+\left[\sqrt{R^{2}+1}-\frac{s^{2}}{2 \sqrt{R^{2}+1}}\right] Y^{1}(\hat{\sigma})\right\} 2 \cosh s Z^{1}(\hat{\sigma}) \mathrm{d} s \\
& \geq e^{-C^{2} / 2} \int_{0}^{C} \exp \left\{\left[\sqrt{R^{2}+1}-\frac{s^{2}}{2 \sqrt{R^{2}+1}}\right] Y^{1}(\hat{\sigma})\right\} \mathrm{d} s .
\end{aligned}
$$

For later convenience in the course of this proof, we will now define

$$
\tilde{Y}^{1}(\hat{\sigma}):=\left[\sqrt{R^{2}+1}-\frac{s^{2}}{2 \sqrt{R^{2}+1}}\right] Y^{1}(\hat{\sigma}) .
$$

We get

$$
\begin{gathered}
P\left(\int_{S_{N}\left(N-R^{2}\right)} \int_{-C}^{C} e^{-\frac{s^{2}}{2}+Z^{1}(\hat{\sigma}) s+\tilde{Y}^{1}(\hat{\sigma})} \mathrm{d} s \mathrm{~d} \nu_{N}^{R} \leq \frac{1}{L}\right) \\
\quad \leq P\left(\int_{S_{N}\left(N-R^{2}\right)} \int_{0}^{C} e^{\tilde{Y}^{1}(\hat{\sigma})} \mathrm{d} s \mathrm{~d} \nu_{N}^{R} \leq \frac{e^{C^{2} / 2}}{L}\right) .
\end{gathered}
$$

Now, by the Cauchy-Schwarz inequality, we derive

$$
\left(\int_{S_{N}\left(N-R^{2}\right)} \int_{0}^{C} e^{\tilde{Y}^{1}(\hat{\sigma})} \mathrm{d} s \mathrm{~d} \nu_{N}^{R}\right)\left(\int_{S_{N}\left(N-R^{2}\right)} \int_{0}^{C} e^{-\tilde{Y}^{1}(\hat{\sigma})} \mathrm{d} s \mathrm{~d} \nu_{N}^{R}\right) \geq C^{2} .
$$

Then, we see

$$
\begin{aligned}
& P\left(\int_{S_{N}\left(N-R^{2}\right)} \int_{0}^{C} e^{\tilde{Y}^{1}(\hat{\sigma})} \mathrm{d} s \mathrm{~d} \nu_{N}^{R} \leq \frac{e^{C^{2} / 2}}{L}\right) \\
& \leq P\left(\int_{S_{N}\left(N-R^{2}\right)} \int_{0}^{C} e^{-\tilde{Y}^{1}(\hat{\sigma})} \mathrm{d} s \mathrm{~d} \nu_{N}^{R} \geq \frac{L C^{2}}{e^{C^{2} / 2}}\right) .
\end{aligned}
$$

Notice that $-Y^{1}(\hat{\sigma})$ and $Y^{1}(\hat{\sigma})$ have the same distribution. We will bound the right hand side of the above equation by Markov's inequality.

Let us find the $k$ th moment; it is

$$
\mathbb{E} \int_{S_{N}\left(N-R^{2}\right) \otimes k} \int_{0}^{C} \ldots \int_{0}^{C} e^{-\sum_{j=1}^{k} \tilde{Y}^{1}\left(\hat{\sigma}_{j}\right)} \mathrm{d} s_{1} \ldots \mathrm{d} s_{k}\left(\mathrm{~d} \nu_{N}^{R}\right)^{\otimes k} .
$$

We can perform the integration in the $\tilde{Y}^{1}\left(\hat{\sigma}_{j}\right)$ Gaussian random variables first. Since $R$ and $s_{i} \in[0, C]$ are bounded, this is certainly some finite quantity. Thus, we can apply Markov's inequality in order to get the bound that

$$
P\left(\int_{S_{N}\left(N-R^{2}\right)} \int_{0}^{C} e^{-\tilde{Y}^{1}(\hat{\sigma})} \mathrm{d} s \mathrm{~d} \nu_{N}^{R} \geq \frac{L C^{2}}{e^{C^{2} / 2}}\right) \leq \frac{K(k)}{L^{k}},
$$

where the constant $K(k)$ that appears depends only on $C, R$ and $k$ in some manner. This establishes the lemma. 
Remark 3.3. Implicit in the proof of the lower bound of probability is a proof of a corresponding upper bound. In equation (3.26), we took a moment bound of the variable $e^{\tilde{Y}^{1}(\hat{\sigma})}$ to derive probability bounds.

One can apply the same strategy in order to bound the probability of being too large. The only minor difference is that one also needs to take moment bounds of the $Z$ variables. However, these are also Gaussians, so the process is similar.

\subsection{The uniform limit in $R$}

Here, we will compute $\lim _{R \rightarrow \infty} F^{R, C, E}(\zeta)$ for those $\zeta$ that satisfy finite replica symmetry breaking. If we then show that if we can bound the difference $\mid F^{R, C, E}(\zeta)-$ $\lim _{R \rightarrow \infty} F^{R, C, E}(\zeta) \mid$ uniformly along the sequence $\zeta_{i}$ approaching $\zeta^{*}$, we will be able to exchange the limit $\lim _{i \rightarrow \infty} \lim _{R \rightarrow \infty} F^{C, R, E}\left(\zeta_{i}\right)=\lim _{R \rightarrow \infty} \lim _{i \rightarrow \infty} F^{C, R, E}\left(\zeta_{i}\right)$.

We can compute the limit $R \rightarrow \infty$ by applying the Bolthausen-Sznitman invariance for RPCs with respect to the Gaussian tilt $\prod_{i=1}^{n} \mathfrak{Y}_{i}\left(h_{\alpha}\right) \sqrt{R^{2}+1}$ in formulas (3.5) and (3.7). For simplicity of presentation, we will only present the computation in the case that $n=1$, which contains the main idea of the proof. The computation for general $n$ is very similar.

Lemma 3.4. Assume we are evaluating $F^{R, C, E}(\zeta)$ so that the overlap distribution $\zeta$ satisfies finite replica symmetry breaking. Let the support of the measure $\zeta$ be $0=q_{0} \leq$ $q_{1} \leq \ldots \leq q_{r}=q_{*}$. We then have the following result

$$
\begin{aligned}
& \lim _{R \rightarrow \infty} \mathbb{E} \prod_{l=1}^{k} \frac{\sum_{\alpha_{l}} w_{\alpha_{l}} \prod_{i=1}^{n} e^{\sqrt{R^{2}+1} \mathfrak{Y}_{i}\left(h_{\alpha_{l}}\right)} \int_{-C}^{C} s_{i}^{e_{l, i}} e^{-\frac{1}{2}\left[1+\hat{b}_{\text {in }}\right] s_{i}^{2}+\tilde{M}_{i}\left(h_{\alpha_{l}}\right)} \mathrm{d} s_{i}}{\sum_{\alpha_{l}} w_{\alpha_{l}} \prod_{i=1}^{n} e^{\sqrt{R^{2}+1} \mathfrak{Y}_{i}\left(h_{\alpha_{l}}\right)} \int_{-C}^{C} e^{-\frac{1}{2}\left[1+\hat{b}_{\text {in }}\right] s_{i}^{2}+\tilde{M}_{i}\left(h_{\alpha_{l}}\right)} \mathrm{d} s_{i}} \\
& =\mathbb{E} \prod_{l=1}^{k} \frac{\sum_{\alpha_{l}} w_{\alpha_{l}} \prod_{i=1}^{n} \int_{-C}^{C} s_{i}^{e_{l, i}} e^{-\frac{1}{2}\left[1+\hat{b}_{\zeta}\right] s_{i}^{2}+\mathfrak{Z}_{i}\left(h_{\alpha_{l}}\right) s_{i}} \mathrm{~d} s_{i}}{\sum_{\alpha_{l}} w_{\alpha_{l}} \prod_{i=1}^{n} \int_{-C}^{C} e^{-\frac{1}{2}\left[1+\hat{b}_{\zeta}\right] s_{i}^{2}+\mathfrak{Z}_{i}\left(h_{\alpha_{l}}\right) s_{i}} \mathrm{~d} s_{i}},
\end{aligned}
$$

where we use $\tilde{M}_{i}\left(h_{\alpha^{l}}\right)$ as a shorthand for the expression

$$
\mathfrak{Z}_{i}\left(h_{\alpha_{l}}\right) s_{i}-\mathfrak{Y}_{i}\left(h_{\alpha_{l}}\right) \frac{s_{i}^{2}}{2 \sqrt{R^{2}+1}}+\frac{\left(\xi^{\prime}(1)-q_{*} \xi^{\prime}\left(q_{*}\right)\right) s_{i}^{4}}{4\left(R^{2}+1\right)}
$$

and $\hat{b}_{\zeta}:=\xi^{\prime}\left(q_{*}\right)\left(1-q_{*}\right)+\sum_{i=1}^{r}\left(q_{i} \xi^{\prime}\left(q_{i}\right)-q_{i-1} \xi^{\prime}\left(q_{i-1}\right)\right) \zeta\left(\left[0, q_{i-1}\right]\right)$.

Moreover, the limit in $R$ is uniform in $\zeta$ for $\zeta_{i}$ a sequence of probability measures with finite replica symmetry breaking that approach $\zeta^{*}$.

Proof. We will illustrate the computation in the single replica, single cavity case with $e_{1,1}=1$. The proof in all other cases will be similar, with marginally more involved computations. Let $N$ denote the numerator of the expression in the first line of (3.27) under these conditions, and let $D$ denote the denominator of said expression. We define $S$ to be $\sum_{\alpha} w_{\alpha} e^{\sqrt{R^{2}+1} \mathfrak{Y}_{1}\left(h_{\alpha}\right)}$. Clearly, we can rewrite the expression in the first line of (3.27) as $\mathbb{E} \frac{N S^{-1}}{D S^{-1}}$. Clearly, we will now be able to compute the quantity better if we understand the tilted Gibbs weight $\frac{w_{\alpha} e^{\sqrt{R^{2}+1} \mathfrak{y}_{1}\left(h_{\alpha}\right)}}{\sum_{\alpha} w_{\alpha} e^{\sqrt{R^{2}+1}} \mathfrak{y}_{1}\left(h_{\alpha}\right)}$.

Since we are just tilting by the exponential of independent Gaussians associated with each $\alpha$, at each level of the Ruelle probability cascade, we apply the standard Bolthausen-Sznitman invariance [5] level by level. This states that if $g_{\alpha}$ are i.i.d. $N(0,1)$ random variables independent of the $u_{\alpha}$, which are the weights of a Poisson-Dirichlet process with parameter $m$, then

$$
\left(e^{-m t^{2} / 2} u_{\alpha} e^{t g_{\alpha}}, g_{\alpha}-m t\right) \stackrel{d}{=}\left(u_{\alpha}, g_{\alpha}\right) .
$$


If one applies the above procedure for our RPC, we shift the Gaussian $\mathfrak{Y}_{1}\left(h_{\alpha}\right)$ by mean equal to $\sqrt{R^{2}+1} \sum_{i=1}^{r}\left(q_{i} \xi^{\prime}\left(q_{i}\right)-q_{i-1} \xi^{\prime}\left(q_{i-1}\right)\right) \zeta\left(\left[0, q_{i-1}\right]\right)$.

Let me describe this in a bit more detail. The weight $w_{\alpha}$ is the normalized product of $r$ Poisson Dirichlet processes, $v_{\alpha^{1}}^{1} v_{\alpha^{2}}^{2} \ldots v_{\alpha^{r}}^{r} . v_{\alpha^{k}}^{k}$ is a Poisson-Dirichlet Process with parameter $\zeta\left[0, q_{k-1}\right]$, and $\alpha^{k}$ denotes the ancestor of $\alpha$ at height $k$ ( $\alpha^{r}$ will just be $\alpha$ itself).

The Gaussian variable $\mathfrak{Y}_{1}\left(h_{\alpha}\right)$ can be decomposed as the sum of $r$ independent Gaussians, $\mathfrak{Y}_{1}\left(h_{\alpha}\right)=\sum_{i=1}^{r} \mathfrak{Y}_{1}^{i}\left(\alpha^{i}\right)$ where $\left.\operatorname{Cov}\left(\mathfrak{Y}_{1}^{i}\left(\alpha^{i}\right), \mathfrak{Y}_{1}\left(\beta^{i}\right)\right)\right)=\delta_{\alpha^{i}, \beta^{i}}\left(q_{i} \xi^{\prime}\left(q_{i}\right)-q_{i-1} \xi^{\prime}\left(q_{i-1}\right)\right)$. We remark here that $\mathfrak{Y}^{i}$ is associated with $\alpha^{i}$ (the nodes that are of depth $i$ on the tree).

We can shift the distribution of each

$$
\begin{aligned}
& \left(v_{\alpha^{k}}^{k} e^{\sqrt{R^{2}+1}} \mathfrak{Y}_{1}^{k}\left(\alpha^{k}\right), \mathfrak{Y}_{1}^{k}\left(\alpha^{k}\right)-\sqrt{R^{2}+1} \zeta\left(\left[0, q_{k-1}\right]\right)\left(q_{k} \xi^{\prime}\left(q_{k}\right)-q_{k-1} \xi^{\prime}\left(q_{k-1}\right)\right)\right) \stackrel{d}{=} \\
& \left(v_{\alpha^{k}}^{k} e^{\frac{\zeta\left(\left[0, q_{k-1}\right]\right)\left(q_{k} \xi^{\prime}\left(q_{k}\right)-q_{k-1} \xi^{\prime}\left(q_{k-1}\right)\right)\left(R^{2}+1\right)}{2}}, \mathfrak{Y}_{1}^{k}\left(\alpha^{k}\right)\right) \text {. }
\end{aligned}
$$

This translates into the replacement,

$$
\begin{aligned}
v_{\alpha^{k}}^{k} e^{\sqrt{R^{2}+1} \mathfrak{Y}_{1}^{k}} e^{-\frac{s^{2}}{2 \sqrt{R^{2}+1}} \mathfrak{Y}_{1}^{k}} \stackrel{d}{=} & v_{\alpha^{k}}^{k} e^{-\frac{s^{2}}{2 \sqrt{R^{2}+1}}\left(\mathfrak{Y}_{1}^{k}+\sqrt{R^{2}+1} \zeta\left(\left[0, q_{k-1}\right]\right)\left(q_{k} \xi^{\prime}\left(q_{k}\right)-q_{k-1} \xi^{\prime}\left(q_{k-1}\right)\right)\right)} \\
& \times e^{\frac{\zeta\left(\left[0, q_{k-1}\right]\right)\left(q_{k} \xi^{\prime}\left(q_{k}\right)-q_{k-1} \xi^{\prime}\left(q_{k-1}\right)\right)\left(R^{2}+1\right)}{2}}
\end{aligned}
$$

We note that the constant rescaling factor $e^{\frac{\zeta\left(\left[0, q_{k}\right]\right)\left(q_{k} \xi^{\prime}\left(q_{k}\right)-q_{k-1} \xi^{\prime}\left(q_{k-1}\right)\right)\left(R^{2}+1\right)}{2}}$ will cancel in both the numerator and the denominator. The only remaining effect is the shift in the mean. Notice, in particular, that we are able to cancel out the prefactors of $\sqrt{R^{2}+1}$ that appear; this is what allows all computations to take a finite value as $R$ goes to $\infty$. To simplify notation, recall $\hat{b}_{\zeta}:=\xi^{\prime}\left(q_{*}\right)\left(1-q_{*}\right)+\sum_{i=1}^{r}\left(q_{r} \xi^{\prime}\left(q_{r}\right)-q_{r-1} \xi^{\prime}\left(q_{r-1}\right)\right) \zeta\left(\left[0, q_{r-1}\right]\right)$.

As a result of applying our distributional equivalence to all the variables $\mathfrak{Y}_{1}^{k}$, we see that the top line of (3.27) is given as

$$
\lim _{R \rightarrow \infty} \mathbb{E} \frac{\sum_{\alpha} w_{\alpha} \int_{-C}^{C} s e^{-\frac{1}{2} s^{2}\left[1+\hat{b}_{\zeta}\right]+\mathfrak{Z}_{1}\left(h_{\alpha}\right) s-\mathfrak{Y}_{1}\left(h_{\alpha}\right) \frac{s^{2}}{2 \sqrt{R^{2}+1}}+\frac{\left(\xi^{\prime}(1)-q_{*} \xi^{\prime}\left(q_{*}\right)\right) s^{4}}{4\left(R^{2}+1\right)}} \mathrm{d} s}{\sum_{\alpha} w_{\alpha} \int_{-C}^{C} e^{-\frac{1}{2} s^{2}\left[1+\hat{b}_{\zeta}\right]+\mathfrak{Z}_{1}\left(h_{\alpha}\right) s-\mathfrak{Y}_{1}\left(h_{\alpha}\right) \frac{s^{2}}{2 \sqrt{R^{2}+1}}+\frac{\left(\xi^{\prime}(1)-q_{*} \xi^{\prime}\left(q_{*}\right)\right) s^{4}}{4\left(R^{2}+1\right)}} \mathrm{d} s} .
$$

We did not replace the quantity inside the exponential with $\tilde{M}$ in order to clearly illustrate the limit procedure. We will show that the above limit is

$$
\mathbb{E}\left[\frac{\sum_{\alpha} w_{\alpha} \int_{-C}^{C} s e^{-\frac{1}{2}\left[1+\hat{b}_{\zeta}\right] s^{2}+\mathfrak{Z}_{1}\left(h_{\alpha}\right) s} \mathrm{~d} s}{\sum_{\alpha} w_{\alpha} \int_{-C}^{C} e^{-\frac{1}{2}\left[1+\hat{b}_{\zeta}\right] s^{2}+\mathfrak{Z}_{1}\left(h_{\alpha}\right) s} \mathrm{~d} s}\right]
$$

and that the above limit can be taken uniformly in $\zeta$.

We denote the numerator of (3.30) as $N_{R}$ and the denominator as $D_{R}$, while we denote the numerator of (3.31) as $N_{\infty}$ and the denominator as $D_{\infty}$.

We will bound the difference

$$
\mathbb{E}\left[\left|\frac{N_{R}}{D_{R}}-\frac{N_{\infty}}{D_{\infty}}\right|\right] \leq \mathbb{E}\left[\left|\frac{N_{R}\left(D_{\infty}-D_{R}\right)}{D_{R} D_{\infty}}\right|+\left|\frac{N_{R}-N_{\infty}}{D_{\infty}}\right|\right] .
$$

We will first bound $\mathbb{E}\left|\frac{N_{R}\left(D_{\infty}-D_{R}\right)}{D_{R} D_{\infty}}\right|$; the bound on the other part will be similar. We first remark that $\frac{N_{R}}{D_{R}} \leq C$ as this is an upper bound on every individual ratio $\frac{\int_{-C}^{C} s e^{-\frac{1}{2}\left[1+\hat{b}_{\zeta}\right] s^{2}+3_{1}\left(h_{\alpha}\right) s} \mathrm{~d} s}{\int_{-C}^{C} e^{-\frac{1}{2}\left[1+\hat{b}_{\zeta}\right] s^{2}+3_{1}\left(h_{\alpha}\right) s} \mathrm{~d} s}$ and each individual term in the denominator is positive. It will now suffice to uniformly bound the quantity $\mathbb{E}\left|\frac{D_{\infty}-D_{R}}{D_{\infty}}\right|$. 
First, notice that we are able to bound

$$
\begin{aligned}
& \left|D_{\infty}-D_{R}\right| \\
& =\left|\sum_{\alpha} w_{\alpha} \int_{-C}^{C} e^{-\frac{1}{2} s^{2}\left[1+\hat{b}_{\zeta}\right]+\tilde{M}\left(h_{\alpha}\right)} \mathrm{d} s-\sum_{\alpha} w_{\alpha} \int_{-C}^{C} e^{-\frac{1}{2}\left[1+\hat{b}_{\zeta}\right] s^{2}+\mathfrak{Z}_{1}\left(h_{\alpha}\right) s} \mathrm{~d} s\right| \\
& =\left|\sum_{\alpha} w_{\alpha} \int_{-C}^{C} e^{-\frac{1}{2} s^{2}\left[1+\hat{b}_{\zeta}\right]+\mathfrak{Z}_{1}\left(h_{\alpha}\right) s}\left[e^{-\mathfrak{Y}_{1}\left(h_{\alpha}\right) \frac{s^{2}}{2 \sqrt{R^{2}+1}}+\frac{\left.\left(\xi^{\prime}(1)-q_{*} \xi^{\prime}\left(q_{*}\right)\right)\right) s^{4}}{4\left(R^{2}+1\right)}}-1\right] \mathrm{d} s\right| \\
& \leq \sum_{\alpha} w_{\alpha} \int_{-C}^{C} e^{-\frac{1}{2}\left[1+\hat{b}_{\zeta}\right] s^{2}+\mathfrak{Z}_{1}\left(h_{\alpha}\right) s} \mathrm{~d} s\left[e^{\left|\mathfrak{Y}_{1}\left(h_{\alpha}\right)\right| \frac{C^{2}}{2 \sqrt{R^{2}+1}}+\frac{\left(\xi^{\prime}(1)-q_{*} \xi^{\prime}\left(q_{*}\right)\right) C^{4}}{4\left(R^{2}+1\right)}}-1\right] .
\end{aligned}
$$

To get from the third line to the fourth line, we took the absolute value inside to the quantity in brackets. Then, we could upper bound this quantity in brackets by setting all terms in the exponential as large as possible(each $s$ to $C$, and bounding $\mathfrak{Y}$ by its absolute value). The quantity in the exponential is positive, so the exponential is greater than 1 . Therefore, the quantity in brackets on the fourth line is positive.

Thus, it suffices to uniformly control

$$
\mathbb{E} \frac{\sum_{\alpha} w_{\alpha} \int_{-C}^{C} e^{-\frac{1}{2}\left[1+\hat{b}_{\zeta}\right] s^{2}+\mathfrak{Z}_{1}\left(h_{\alpha}\right) s} \mathrm{~d} s\left[e^{\mid \mathfrak{Y})_{1}\left(h_{\alpha}\right) \mid \frac{C^{2}}{2 \sqrt{R^{2}+1}}+\frac{\left(\xi^{\prime}(1)-q_{*} \xi^{\prime}\left(q_{*}\right)\right) C^{4}}{4\left(R^{2}+1\right)}}-1\right]}{\sum_{\alpha} w_{\alpha} \int_{-C}^{C} e^{-\frac{1}{2}\left[1+\hat{b}_{\zeta}\right] s^{2}+\mathfrak{Z}_{1}\left(h_{\alpha}\right) s} \mathrm{~d} s} .
$$

Since the $\mathfrak{Y}_{1}$ variables are independent of anything else that appears in the denominator or numerator, we can take the expectation of these first. The expression above will then factor as a product like

$$
\mathbb{E}_{\mathfrak{Y}_{1}}\left[e^{\left|\mathfrak{Y}_{1}\right| \frac{C^{2}}{2 \sqrt{R^{2}+1}}+\frac{\left(\xi^{\prime}(1)-q_{*} \xi^{\prime}\left(q_{*}\right)\right) C^{4}}{4\left(R^{2}+1\right)}}-1\right] \mathbb{E} \frac{\sum_{\alpha} w_{\alpha} \int_{-C}^{C} e^{-\frac{1}{2}\left[1+\hat{b}_{\zeta}\right] s^{2}+\mathfrak{Z}_{1}\left(h_{\alpha}\right) s} \mathrm{~d} s}{\sum_{\alpha} w_{\alpha} \int_{-C}^{C} e^{-\frac{1}{2}\left[1+\hat{b}_{\zeta}\right] s^{2}+\mathfrak{3}_{1}\left(h_{\alpha}\right) s} \mathrm{~d} s} .
$$

Clearly, the second expectation considered in the expression above is 1 . We can bound the above expression by the $L_{1}$ norm of the function $e^{|y| \frac{C^{2}}{2 \sqrt{R^{2}+1}}+\frac{\left(\xi^{\prime}(1)-q_{*} \xi^{\prime}\left(q_{*}\right)\right) C^{4}}{4\left(R^{2}+1\right)}}-1$ where $y$ is a Gaussian with variance $q_{*} \xi^{\prime}\left(q_{*}\right)$. This clearly goes to 0 as $R$ goes to $\infty$, and we have derived the infinite limit. We remark here that the rate of convergence only depends on $C$ and the covariance function $\xi$; it does not depend on the specific finite replica symmetry breaking model chosen.

Now, we consider the difference $\mathbb{E}\left[\frac{N_{R}-N_{\infty}}{D_{\infty}}\right]$. We can write the numerator as

$$
\begin{aligned}
& \left|N_{R}-N_{\infty}\right| \\
& =\left|\sum_{\alpha} w_{\alpha} \int_{-C}^{C} s e^{-\frac{1}{2} s^{2}\left[1+\hat{b}_{\zeta}\right]+\tilde{M}\left(h_{\alpha}\right)} \mathrm{d} s-\sum_{\alpha} w_{\alpha} \int_{-C}^{C} s e^{-\frac{1}{2}\left[1+\hat{b}_{\zeta}\right] s^{2}+\mathfrak{Z}_{1}\left(h_{\alpha}\right) s} \mathrm{~d} s\right| \\
& =\left|\sum_{\alpha} w_{\alpha} \int_{-C}^{C} e^{-\frac{1}{2} s^{2}\left[1+\hat{b}_{\zeta}\right]+\mathfrak{Z}_{1}\left(h_{\alpha}\right) s}\left[s e^{-\mathfrak{Y}_{1}\left(h_{\alpha}\right) \frac{s^{2}}{2 \sqrt{R^{2}+1}}+\frac{\left.\left(\xi^{\prime}(1)-q_{*} \xi^{\prime}\left(q_{*}\right)\right)\right) s^{4}}{4\left(R^{2}+1\right)}}-1\right] \mathrm{d} s\right| \\
& \leq C \sum_{\alpha} w_{\alpha} \int_{-C}^{C} e^{-\frac{1}{2}\left[1+\hat{b}_{\zeta}\right] s^{2}+\mathfrak{Z}_{1}\left(h_{\alpha}\right) s} \mathrm{~d} s\left[e^{\left|\mathfrak{Y}_{1}\left(h_{\alpha}\right)\right| \frac{C^{2}}{2 \sqrt{R^{2}+1}}+\frac{\left(\xi^{\prime}(1)-q_{*} \xi^{\prime}\left(q_{*}\right)\right) C^{4}}{4\left(R^{2}+1\right)}}-1\right] .
\end{aligned}
$$

As before, to get from the third line to the fourth line, we take the absolute value inside, onto the quantity in brackets, and maximize all possible expressions. Now, this includes bounding the $s$ outside the exponential but inside the brackets by $C$. Aside from the fixed factor of $C$ outside, the quantity on the last line is the same as that appearing in (3.33), and the analysis that follows would be the same. 
Remark 3.5. We remark here that the constant $\hat{b}_{\zeta}$ is slightly different from the constant $b_{\zeta}$ that appears in equation (1.14). What is true is that $\hat{b}_{\zeta}$, as defined here, is equal to the constant that appears in the denominator of $u^{\zeta}\left(q_{*}, x\right)$ for solutions to the Parisi initial value problem (A.21) with initial data $u^{\zeta}(1, x)=\frac{x^{2}}{2\left(1+b_{\zeta}\right)}$. We expect this since the highest overlap level we consider in our finite RSB model is $q_{*}$.

Recalling Corollary 2.8, we see we have established the following statement with the above lemma.

$$
\lim _{N \rightarrow \infty} \mathbb{E} \prod_{l=1}^{k}\left\langle\left(\sigma_{1}^{l}\right)^{e_{1, l}} \ldots\left(\sigma_{n}^{l}\right)^{e_{l, n}}\right\rangle_{H_{N+n}\left(S_{N+n}\right)}=\lim _{C \rightarrow \infty} \lim _{i \rightarrow \infty} \lim _{R \rightarrow \infty} F^{R, C, E}\left(\zeta_{i}\right) .
$$

Since each $\zeta_{i}$ satisfies finite replica symmetry breaking, the limit

$$
F^{C, E}\left(\zeta_{i}\right)=\mathbb{E} \prod_{l=1}^{k}\left[\frac{\sum_{\alpha_{l}} w_{\alpha_{l}} \prod_{i=1}^{n} \int_{-C}^{C} s_{i}^{e_{l, i}} e^{-\frac{1}{2}\left[1+\hat{b}_{\zeta}\right] s_{i}^{2}+\mathfrak{Z}_{i}\left(h_{\alpha_{l}}\right) s_{i}} \mathrm{~d} s_{i}}{\sum_{\alpha_{l}} w_{\alpha_{l}} \prod_{i=1}^{n} \int_{-C}^{C} e^{-\frac{1}{2}\left[1+\hat{b}_{\zeta}\right] s_{i}^{2}+\mathfrak{Z}_{i}\left(h_{\alpha_{l}}\right) s_{i}} \mathrm{~d} s_{i}}\right],
$$

is the value of $\lim _{R \rightarrow \infty} F^{R, C, E}\left(\zeta_{i}\right)$, where the $w_{\alpha}$ are the weights of some appropriate RPC.

\subsection{The $C \rightarrow \infty$ limit}

There is a natural guess for the $C \rightarrow \infty$ limit. It suffices to show that this limit exists and is uniform in the variable $\zeta$.

Lemma 3.6. Assume we are evaluating $F^{C, E}(\zeta)$ so that the overlap distribution $\zeta$ satisfies finite replica symmetry breaking. Let the support of the measure $\zeta$ be $0=q_{0} \leq q_{1} \leq$ $\ldots \leq q_{r}=q_{*}$. We then have the following result

$$
\begin{aligned}
& \lim _{C \rightarrow \infty} \mathbb{E}\left[\prod_{l=1}^{k} \frac{\sum_{\alpha_{l}} w_{\alpha_{l}} \prod_{i=1}^{n} \int_{-C}^{C} s_{i}^{e_{l, i}} e^{-\frac{1}{2}\left[1+\hat{b}_{\zeta}\right] s_{i}^{2}+3_{i}\left(h_{\alpha_{l}}\right) s_{i}} \mathrm{~d} s_{i}}{\sum_{\alpha_{l}} w_{\alpha_{l}} \prod_{i=1}^{n} \int_{-C}^{C} e^{-\frac{1}{2}\left[1+\hat{b}_{\zeta}\right] s_{i}^{2}+3_{i}\left(h_{\alpha_{l}}\right) s_{i}} \mathrm{~d} s_{i}}\right] \\
& =\mathbb{E} \prod_{l=1}^{k}\left[\frac{\sum_{\alpha_{l}} w_{\alpha_{l}} \prod_{i=1}^{n} \int_{-\infty}^{\infty} s_{i}^{e_{l, i}} e^{-\frac{1}{2}\left[1+\hat{b}_{\zeta}\right] s_{i}^{2}+3_{i}\left(h_{\alpha}\right) s_{i}} \mathrm{~d} s_{i}}{\sum_{\alpha_{l}} w_{\alpha_{l}} \prod_{i=1}^{n} \int_{-\infty}^{\infty} e^{-\frac{1}{2}\left[1+\hat{b}_{\zeta}\right] s_{i}^{2}+\mathfrak{3}_{i}\left(h_{\alpha_{l}}\right) s_{i}} \mathrm{~d} s_{i}}\right] \\
& =\mathbb{E} \prod_{l=1}^{k}\left[\frac{\sum_{\alpha_{l}} w_{\alpha_{l}} \prod_{i=1}^{n} \rho_{\zeta, h_{\alpha_{l}}}^{3_{i}}\left(s^{e_{l, i}}\right) e^{\frac{\left(\mathfrak { 3 } _ { i } \left(h_{\left.\left.\alpha_{l}\right)\right)^{2}}^{2\left[1+\hat{b}_{\zeta}\right]}\right.\right.}{\left.2\left(h_{\alpha_{l}}\right)\right)^{2}}}}{\sum_{\alpha_{l}} w_{\alpha_{l}} \prod_{i=1}^{n} e^{\frac{\left(3_{i}\right.}{2\left[1+\hat{b}_{\zeta}\right]}}}\right] \\
& =\mathbb{E} \prod_{l=1}^{k}\left[\sum_{\alpha_{l}} w_{\alpha_{l}} \prod_{i=1}^{n} \rho_{\zeta, h_{\alpha_{l}}}^{\left(\mathfrak{Z}_{i}\right)^{\prime}}\left(s^{e_{l, i}}\right)\right] .
\end{aligned}
$$

The transition between the second and third lines is the definition of $\rho_{\zeta, h_{\alpha}}^{3_{i}}$, as in (1.16). The transition between the third and fourth lines is due to the the construction of the random variables $\left(\mathfrak{Z}_{i}\right)^{\prime}$ is as given in the appendix $A$.

Moreover, the limit in $C$ can be taken uniformly in $\zeta$ for a sequence of finite replica symmetry breaking measures approaching $\zeta^{*}$.

Proof. We will show this computation in the case that $n=1, k=1$ and $e_{1,1}=1$. More general $E$ can be done using similar computations. Let $N_{C}$ denote the numerator

$$
N_{C}:=\sum_{\alpha} w_{\alpha} \int_{-C}^{C} s e^{-\frac{1}{2}\left[1+\hat{b}_{\zeta}\right] s^{2}+\mathfrak{\jmath}_{1}\left(h_{\alpha}\right) s} \mathrm{~d} s
$$


and let $D_{C}$ denote the denominator

$$
D_{C}:=\sum_{\alpha} w_{\alpha} \int_{-C}^{C} e^{-\frac{1}{2}\left[1+\hat{b}_{\zeta}\right] s^{2}+\mathfrak{Z}_{1}\left(h_{\alpha}\right) s} \mathrm{~d} s .
$$

Correspondingly, let $N_{\infty}$ be the numerator of the infinite limit

$$
N_{\infty}:=\sum_{\alpha} w_{\alpha} \int_{-\infty}^{\infty} s e^{-\frac{1}{2}\left[1+\hat{b}_{\zeta}\right] s^{2}+\mathfrak{Z}_{1}\left(h_{\alpha}\right) s} \mathrm{~d} s,
$$

and $D_{\infty}$ be the denominator of the infinite limit

$$
D_{\infty}:=\sum_{\alpha} w_{\alpha} \int_{-\infty}^{\infty} e^{-\frac{1}{2}\left[1+\hat{b}_{\zeta}\right] s^{2}+\mathfrak{Z}_{1}\left(h_{\alpha}\right) s} \mathrm{~d} s .
$$

As before, we will bound

$$
\mathbb{E}\left|\frac{N_{C}}{D_{C}}-\frac{N_{\infty}}{D_{\infty}}\right| \leq \mathbb{E}\left|\frac{N_{C}\left|D_{\infty}-D_{C}\right|}{D_{C} D_{\infty}}\right|+\mathbb{E}\left|\frac{\left|N_{\infty}-N_{C}\right|}{D_{\infty}}\right| .
$$

We will first control the value of the former quantity. We first bound $\frac{N_{C}}{D_{C}}$ by C. We see that then it would suffice to show that $\mathbb{E}\left|\frac{D_{\infty}-D_{C}}{D_{\infty}}\right|$ decays at a rate faster than $\frac{1}{C}$. Let $G_{C}(x)$ be the function $\frac{\int_{(-\infty, \infty) \backslash[-C, C]} e^{-\frac{1}{2}\left[1+\hat{b}_{\zeta}\right] s^{2}+x s} \mathrm{~d} s}{\int_{(-\infty, \infty)} e^{-\frac{1}{2}\left[1+\hat{b}_{\zeta}\right] s^{2}+x s} \mathrm{~d} s}$; let $F(x)$ be the function $\int_{-\infty}^{\infty} e^{-\frac{1}{2}\left[1+\hat{b}_{\zeta}\right] s^{2}+x s} \mathrm{~d} s$.

We see that we can then write $\mathbb{E}\left|\frac{D_{\infty}-D_{C}}{D_{\infty}}\right|$ as $\mathbb{E} \frac{\sum_{\alpha} w_{\alpha} G_{C}\left(\mathfrak{Z}_{1}\left(h_{\alpha}\right)\right) F\left(\mathfrak{Z}_{1}\left(h_{\alpha}\right)\right)}{\sum_{\alpha} w_{\alpha} F\left(\mathfrak{Z}_{1}\left(h_{\alpha}\right)\right)}$. We apply the modified Bolthausen-Sznitman invariance [12][Chapter 4.4] principle to $\left(w_{\alpha} F\left(\mathfrak{Z}_{1}\left(h_{\alpha}\right)\right)\right)$ to see that the expectation is equal to $\mathbb{E} \sum_{\alpha} w_{\alpha} G_{C}\left(\mathfrak{Z}_{1}^{\prime}\left(h_{\alpha}\right)\right)=\mathbb{E} G_{C}\left(\mathfrak{z}^{\prime}\right)$ for some random variables $\mathfrak{Z}_{1}^{\prime}\left(h_{\alpha}\right)$. $\mathfrak{z}^{\prime}$ is a random variable with the distribution of a single $\mathfrak{Z}_{1}^{\prime}\left(h_{\alpha}\right)$. The random variables $\mathfrak{Z}_{1}^{\prime}\left(h_{\alpha}\right)$ have been constructed in Appendix A.

We discuss some properties of the function $G_{C}(x)$. First, one can observe that the function $G_{C}(x)$ is always less than 1. Furthermore, $G_{C}(x)$ can be shown to be exponentially small in $C$ as long as $x$ is relatively small compared to $C$. Consider the following,

$$
\begin{aligned}
& G_{C}(x)=\frac{\int_{C}^{\infty} e^{-\frac{1}{2}\left[1+\hat{b}_{\zeta}\right]\left[s-\frac{x}{1+\hat{b}_{\zeta}}\right]^{2}+\frac{x^{2}}{2\left(1+\hat{b}_{\zeta}\right)}} \mathrm{d} s+\int_{-\infty}^{-C} e^{-\frac{1}{2}\left[1+\hat{b}_{\zeta}\right]\left[s-\frac{x}{1+\hat{b}_{\zeta}}\right]^{2}+\frac{x^{2}}{2\left(1+\hat{b}_{\zeta}\right)}} \mathrm{d} s}{\int_{-\infty}^{\infty} e^{-\frac{1}{2}\left[1+\hat{b}_{\zeta}\right]\left[s-\frac{x}{1+\hat{b}_{\zeta}}\right]^{2}+\frac{x^{2}}{2\left(1+\hat{b}_{\zeta}\right)}} \mathrm{d} s} \\
&=\frac{\int_{C-\frac{x}{1+\hat{b}_{\zeta}}}^{\infty} e^{-\frac{1}{2}\left[1+\hat{b}_{\zeta}\right] s^{2}} \mathrm{~d} s+\int_{\infty}^{-C-\frac{x}{1+\hat{b}_{\zeta}}} e^{-\frac{1}{2}\left[1+\hat{b}_{\zeta}\right] s^{2}} \mathrm{~d} s}{\int_{-\infty}^{\infty} e^{-\frac{1}{2}\left[1+\hat{b}_{\zeta}\right] s^{2}} \mathrm{~d} s} \\
& \leq \frac{\int_{C-\frac{x}{1+\hat{b}_{\zeta}}}^{\infty} s e^{-\frac{1}{2}\left[1+\hat{b}_{\zeta}\right] s^{2}} \mathrm{~d} s+\int_{\infty}^{-C-\frac{x}{1+\hat{b}_{\zeta}}}|s| e^{-\frac{1}{2}\left[1+\hat{b}_{\zeta}\right] s^{2}} \mathrm{~d} s}{\int_{-\infty}^{\infty} e^{-\frac{1}{2}\left[1+\hat{b}_{\zeta}\right] s^{2} \mathrm{~d} s}} \\
& \leq K e^{-\frac{1}{2}\left[1+\hat{b}_{\zeta}\right]\left(C-\frac{x}{1+\hat{b}_{\zeta}}\right)^{2}}+K e^{-\frac{1}{2}\left[1+\hat{b}_{\zeta}\right]\left(-C-\frac{x}{1+\hat{b}_{\zeta}}\right)^{2}} .
\end{aligned}
$$

Here, $K$ is a constant that does not depend on $C$ or $\zeta$. To get from the second line to the third line, we assume that $C-\frac{x}{1+\hat{b}_{\zeta}}>1$ and $-C-\frac{x}{1+\hat{b}_{\zeta}}<-1$. Using the fact that $1+\hat{b}_{\zeta}>1$, we see that both of these conditions can hold simultaneously provided $C$ is sufficiently large and $|x| \leq \frac{C}{2}$. Under these conditions, we have the bound $G_{C}(x) \leq K e^{-\frac{C^{2}}{8}}$. 
We denote $\chi$ to be the characteristic function of the interval complement $\left[\frac{-C}{2}, \frac{C}{2}\right]^{c}$. This gives us the inequality,

$$
\mathbb{E}\left[G_{C}\left(\mathfrak{z}^{\prime}\right)\right] \leq \mathbb{E}\left[\chi\left(\mathfrak{z}^{\prime}\right)\right]+K \mathbb{E}\left[\left(1-\chi\left(\mathfrak{z}^{\prime}\right)\right) e^{-C^{2} / 8}\right],
$$

where we used the fact that $G_{C}$ can be bounded by 1 when $\chi=1$.

Through the probability bounds detailed in Lemma A.3, we see that $\mathbb{E}\left[\left(\mathfrak{z}^{\prime}\right)^{2 p}\right] \leq K$ for some constant that does not depend on $\zeta$ or $C$. By Markov's inequality, this gives us $\mathbb{E}\left[\chi\left(\mathfrak{z}^{\prime}\right)\right] \leq K C^{-2 p}$. For sufficiently large $C$, we also know that $e^{-C^{2} / 8} \leq C^{-2 p}$. Combining these two points gives us the estimate,

$$
\mathbb{E}\left[G_{C}\left(\mathfrak{z}^{\prime}\right)\right] \leq K C^{-2 p},
$$

where the constant $K$ does not depend on $\zeta$ or $C$. This gives us the uniform decay in $\zeta$ for $C \mathbb{E}\left[G_{C}\left(\zeta^{\prime}\right)\right]$ in the $C \rightarrow \infty$ limit.

For the quantity,

$$
\left|\mathbb{E}\left[\frac{N_{\infty}-N_{C}}{D_{\infty}}\right]\right| \leq \mathbb{E}\left[\frac{\sum_{\alpha} w_{\alpha} \int_{(-\infty, \infty) \backslash[-C, C]}|s| e^{-\frac{1}{2}\left[1+\hat{b}_{\zeta}\right] s^{2}+\mathfrak{Z}_{1}\left(h_{\alpha}\right) s} \mathrm{~d} s}{\sum_{\alpha} w_{\alpha} \int_{-\infty}^{\infty} e^{-\frac{1}{2}\left[1+\hat{b}_{\zeta}\right] s^{2}+3_{1}\left(h_{\alpha}\right) s} \mathrm{~d} s}\right],
$$

we can apply a similar logic with the application of the Bolthausen-Sznitman invariance principle to the function $F\left(\mathfrak{Z}\left(h_{\alpha}\right)\right)$. We introduce the function,

$$
G_{C}^{1}(x)=\frac{\int_{(-\infty, \infty) \backslash[-C, C]}|s| e^{-\frac{1}{2}\left[1+\hat{b}_{\zeta}\right] s^{2}+x s} \mathrm{~d} s}{\int_{-\infty}^{\infty} e^{-\frac{1}{2}\left[1+\hat{b}_{\zeta}\right] s^{2}+x s} \mathrm{~d} s} .
$$

After applying the previously mentioned invariance principle, we again see that,

$$
\left|\mathbb{E}\left[\frac{N_{\infty}-N_{C}}{D_{\infty}}\right]\right| \leq \mathbb{E}\left[G_{C}^{1}\left(\mathfrak{z}^{\prime}\right)\right] .
$$

We now derive estimates similar to those of $G_{C}(x)$. By rescaling to the appropriate mean, we see that

$$
\begin{aligned}
G_{C}^{1}(x) & =\frac{\int_{C-\frac{x}{1+\hat{b}_{\zeta}}}^{\infty}\left[s+\frac{x}{1+\hat{b}_{\zeta}}\right] e^{-\frac{1}{2}\left[1+\hat{b}_{\zeta}\right] s^{2}} \mathrm{~d} s+\int_{-\infty}^{-C-\frac{x}{1+\hat{b}_{\zeta}}}\left[-s-\frac{x}{1+\hat{b}_{\zeta}}\right] e^{-\frac{1}{2}\left[1+\hat{b}_{\zeta}\right] s^{2}} \mathrm{~d} s}{\int_{-\infty}^{\infty} e^{-\frac{1}{2}\left[1+\hat{b}_{\zeta}\right] s^{2}} \mathrm{~d} s} \\
& \leq \frac{1}{\sqrt{2 \pi\left[1+\hat{b}_{\zeta}\right]}}\left[e^{-\frac{1}{2}\left[1+\hat{b}_{\zeta}\right]\left[C-\frac{x}{1+\hat{b}_{\zeta}}\right]^{2}}+e^{\left.-\frac{1}{2}\left[1+\hat{b}_{\zeta}\right]\left[-C-\frac{x}{1+\hat{b}_{\zeta}}\right]^{2}\right]}\right. \\
& +\frac{|x|}{1+\hat{b}_{\zeta}} \frac{\int_{(-\infty, \infty) \backslash\left(-C-\frac{x}{1+\hat{b}_{\zeta}}, C-\frac{x}{1+\hat{b}_{\zeta}}\right.} e^{-\frac{1}{2}\left[1+\hat{b}_{\zeta}\right] s^{2}} \mathrm{~d} s}{\int_{-\infty}^{\infty} e^{-\frac{1}{2}\left[1+\hat{b}_{\zeta}\right] s^{2}} \mathrm{~d} s} .
\end{aligned}
$$

To get to the second line, we evaluated the portion of the integrals of the form $s e^{-\frac{1}{2}\left[1+\hat{b}_{\zeta}\right] s^{2}} \mathrm{~d} s$ and applied absolute values to the constant factors $\frac{x}{1+\hat{b}_{\zeta}}$.

The final ratio of integrals appears in the computation of $G_{C}(x)$. The results from there say that when $|x| \leq \frac{C}{2}$, we may be able to bound $G_{C}^{1}(x)$ by $K C e^{-C^{2} / 8}$ for sufficiently large $C$, where $K$ is a constant that does not depend on $C$ or $\zeta$. When $|x| \geq \frac{C}{2}$, we may instead bound the ratio by 1 and instead derive the bound $G_{C}^{1} \leq K x$, where $K$ is a constant that does not depend on $C$ or $\zeta$.

To bound $\mathbb{E}\left[G_{C}^{1}\left(\mathfrak{z}^{\prime}\right)\right]$, we first split based on whether $\left|\mathfrak{z}^{\prime}\right| \leq \frac{C}{2}$. In the region $\left|\mathfrak{z}^{\prime}\right| \geq \frac{C}{2}$, we apply a dyadic decomposition. We see 


$$
\mathbb{E}\left[G_{C}^{1}\left(\mathfrak{z}^{\prime}\right)\right] \leq K C e^{-C^{2} / 8} \mathbb{P}\left[\left|\mathfrak{z}^{\prime}\right| \leq \frac{C}{2}\right]+\sum_{k=0}^{\infty} \sup _{x \in\left[2^{k-1} C, 2^{k} C\right]} G_{C}(x) \mathbb{P}\left[\mathfrak{z}^{\prime} \geq 2^{k-1} C\right] .
$$

By our second moment bound from Lemma A.3, we see that there is a constant $K$ that does not depend on $\zeta$ such that $\mathbb{E}\left[\mathfrak{z}^{\prime} \geq 2^{k-1} C\right] \leq \frac{K}{2^{2 k-2} C^{2}}$. Applying the bound $G_{C}(x) \leq K x$, we can thus bound our dyadic sum by

$$
\sum_{k=0}^{\infty} \sup _{x \in\left[2^{k-1} C, 2^{k} C\right]} G_{C}(x) \mathbb{P}\left[\mathfrak{z}^{\prime} \geq 2^{k-1} C\right] \leq \sum_{k=0}^{\infty} K 2^{k} C \frac{1}{2^{2 k-2} C^{2}} \leq K C^{-1} .
$$

We remark again that this constant does not depend on $C$ or on $\zeta$.

This argument shows that

$$
\lim _{C \rightarrow \infty} \mathbb{E}\left|\frac{N_{C}}{D_{C}}-\frac{N_{\infty}}{D_{\infty}}\right|=0
$$

where the rate of convergence is independent of $\zeta$.

Remark 3.7. If one were to consider higher moments of $s$, say $|s|^{p}$, then one will apply the same version of the modified Bolthausen-Sznitman transformation. The only difference here is that one would need to consider expectations of the form $\mathbb{E}\left[G_{C}^{p}\left(\mathfrak{z}^{\prime}\right)\right]$, where

$$
G_{C}^{p}(x):=\frac{\int_{(-\infty, \infty) \backslash(-C, C)} s^{p} e^{-\frac{1}{2}\left[1+\hat{b}_{\zeta}\right] s^{2}+x s} \mathrm{~d} s}{\int_{-\infty}^{\infty} e^{-\frac{1}{2}\left[1+\hat{b}_{\zeta}\right] s^{2}+x s} \mathrm{~d} s} .
$$

One could show that,

$$
\begin{array}{r}
G_{C}^{p}(x) \leq K|x|^{p},|x| \geq \frac{C}{2}, \\
G_{C}^{p}(x) \leq K C^{p} e^{-C^{2} / 8},|x| \leq \frac{C}{2},
\end{array}
$$

through largely the same computations outlined in the proof. The high moment bounds in Lemma A. 3 would be sufficient to compensate for the increased powers in $x$. The strategy for bounding $\mathbb{E}\left[G_{C}^{p}\left(\mathfrak{z}^{\prime}\right)\right]$ would again follow the strategy outlined in the proof.

To summarize again what we have established, we have

$$
\lim _{N \rightarrow \infty} \mathbb{E} \prod_{l=1}^{k}\left\langle\left(\sigma_{1}^{l}\right)^{e_{l, 1}} \ldots\left(\sigma_{n}^{l}\right)^{e_{l, n}}\right\rangle_{H_{N+n}\left(S_{N+n}\right)}=\lim _{i \rightarrow \infty} \lim _{C \rightarrow \infty} \lim _{R \rightarrow \infty} F^{R, C, E}\left(\zeta_{i}\right),
$$

and that when $\zeta_{i}$ satisfies finite replica symmetry breaking, we have the explicit expression

$$
\lim _{C \rightarrow \infty} \lim _{R \rightarrow \infty} F^{R, C, E}\left(\zeta_{m}\right)=\mathbb{E} \prod_{l=1}^{k}\left[\sum_{\alpha_{l}} w_{\alpha_{l}} \prod_{i=1}^{n} \rho_{\zeta_{m}, h_{\alpha_{l}}}^{\left(3_{i}\right)^{\prime}\left(h_{\alpha_{l}}\right)}\left(s^{e_{l, i}}\right)\right],
$$

where the $w_{\alpha}$ and $\left(\mathfrak{Z}_{i}\right)^{\prime}$ are calculated with respect to an RPC with parameters coming from $\zeta_{i}$. Our final step is to give a succinct representation as $i \rightarrow \infty$.

\section{The local field representation of the spin distribution}

We showed in the previous section that both we can establish cavity equations for the sphere by taking an appropriate limit in $R$ and $C$ and that this expression is continuous in $\zeta$ in the weak topology on measures. In this section, we will use the Cole-Hopf solution 
of the Parisi PDE to determine an expression for the limit in terms of our local field process from Theorem 1.2. The discussion will be similar to [4], but we have to adapt our estimates to the study of the spherical Parisi PDE. Many of our computations are postponed to the Appendix.

Theorem 4.1. Consider a spherical spin glass $H_{N}$ whose limiting overlap distribution is given by $\zeta^{*}$ with $\sup \operatorname{supp}\left(\zeta^{*}\right)=q_{*}$ and limiting Gibbs measure $\nu$. Then, we have that

$$
\lim _{N \rightarrow \infty} \mathbb{E}\left[\prod_{l=1}^{k}\left\langle\prod_{i=1}^{n}\left(\sigma_{i}^{l}\right)^{e_{l, i}}\right\rangle_{H_{N}}\right]=\mathbb{E}\left[\prod_{l=1}^{k} \prod_{i=1}^{n} \rho_{\zeta, \sigma^{l}}^{\mathcal{X}_{q *}^{i}}\left(s^{e_{l, i}}\right)\right],
$$

where the second expectation is taken with respect to the randomness of the process $\mathcal{X}$, and $\sigma^{l}$ are distributed i.i.d from $\nu^{\otimes \infty}$ as in Theorem 1.2.

We obtain Theorem 1.2 as an immediate corollary of this result. One can show equality of the distributions in Theorem 1.2 by explicitly computing the polynomial moments and showing the equality of these moments. Theorem 4.1 computes these polynomial moments and shows equality.

In the previous section, we have related the computations of the spin distributions to computing the distribution of the random variables $\mathfrak{Z}$ and $\mathfrak{Z}^{\prime}$. We start our proof by relating these random variables with the PDE processes defined before Theorem 1.2, assuming that the measure $\zeta$ considered satisfies finite replica symmetry breaking.

Lemma 4.2. Consider a measure $\zeta$ satisfying finite replica symmetry breaking and consider $\mathfrak{Z}\left(h_{\alpha}\right)$ from (3.6), the tilted function $\mathfrak{Z}^{\prime}\left(h_{\alpha}\right)$ as defined in Appendix A, and the associated PDE quantities $\mathcal{Z}, \mathcal{X}$ from (1.12) and (1.15). We have the following equality in distribution

$$
\begin{aligned}
& \left(\mathfrak{Z}\left(h_{\alpha}\right)\right)_{\alpha} \stackrel{d}{=}\left(\mathcal{Z}_{q_{*}}\left(h_{\alpha}\right)\right)_{\alpha}, \\
& \left(\mathfrak{Z}\left(h_{\alpha}\right)^{\prime}\right)_{\alpha} \stackrel{d}{=}\left(\mathcal{X}_{q_{*}}\left(h_{\alpha}\right)\right)_{\alpha} .
\end{aligned}
$$

Proof. The independent increments property of the Brownian motion will give us the first equality. We will now devote ourselves to proving the second equality. For simplicity of presentation, we will show the equivalence for a fixed $h_{\alpha}$. We will use Girsanov's theorem [16][Lemma 6.4.1] to determine the law of $\mathcal{X}_{t}$ at $h_{\alpha}$. Let $Q$ be a measure space in which we have defined the Brownian motion (1.11). Consider a measure $P$ with Radon-Nikodym derivative

$$
\frac{\mathrm{d} P}{\mathrm{~d} Q}=e^{\int_{0}^{t} \zeta([0, s]) \mathrm{d} u^{\zeta}\left(s, \mathcal{Z}_{s}\right)} .
$$

Then under the measure $P$, the law of $\mathcal{Z}_{t}$ is distributed according to the law of $\mathcal{X}_{t}$ under $Q$. In the notation of [16][Lemma 6.4.1], the process $\mathcal{Z}$ satisfies $a(t)=\xi^{\prime \prime}(t)$ and $b(t)=0$. We choose the function $c(t, x)=\zeta([0, t]) u_{x}^{\zeta}(t, x)$. The Cameron-Martin-Girsanov exponential is

$$
R(t):=e^{\int_{0}^{t} \zeta([0, s]) u_{x}^{\zeta}\left(s, \mathcal{Z}_{s}\right) \mathrm{d} \mathcal{Z}_{s}-\frac{1}{2} \int_{0}^{t}(\zeta([0, s]))^{2}\left(u_{x}^{\zeta}\left(s, \mathcal{Z}_{s}\right)\right)^{2} \xi^{\prime \prime}(s) \mathrm{d} s .}
$$

By applying Ito's Lemma and using the equation of the Parisi Initial Value Problem (1.13), $R(t)$ will be the same as the Radon-Nikodym derivative (4.3) defined earlier.

Considering a finite set of times $q_{0}, \ldots, q_{r}$ we have

$$
\begin{aligned}
\mathbb{E}_{Q}\left[F\left(\mathcal{X}_{q_{0}}, \ldots, \mathcal{X}_{q_{r}}\right)\right] & =\mathbb{E}_{P}\left[F\left(\mathcal{Z}_{q_{0}}, \ldots, \mathcal{Z}_{q_{r}}\right)\right] \\
& =\int F\left(\mathcal{Z}_{q_{0}}, \ldots, \mathcal{Z}_{q_{r}}\right) e^{\int_{0}^{t} \zeta([0, s]) \mathrm{d} u^{\zeta}\left(s, \mathcal{Z}_{s}\right)} \mathrm{d} Q(\mathcal{Z}) \\
& =\int F\left(\mathcal{Z}_{q_{0}}, \ldots, \mathcal{Z}_{q_{r}}\right) \prod_{i=1}^{r} e^{\zeta\left(\left[0, q_{k-1}\right]\right)\left(u^{\zeta}\left(q_{k}, \mathcal{Z}_{q_{k}}\right)-u^{\zeta}\left(q_{k-1}, \mathcal{Z}_{q_{k-1}}\right)\right)} \mathrm{d} Q .
\end{aligned}
$$


Now, we use the fact that the Cole-Hopf solution to the Parisi initial value problem is given by $u^{\zeta}\left(q_{k}, \mathcal{Z}_{q_{k}}\right)=X_{k}\left(\mathcal{Z}_{q_{1}}-\mathcal{Z}_{q_{0}}, \ldots, \mathcal{Z}_{q_{k}}-\mathcal{Z}_{q_{k-1}}\right)$ up to a normalization constant (one can refer to the construction in the Appendix), and we have finished the proof.

Now, we cite a weak continuity result from [4] regarding the behavior of the right hand side of (4.1).

Let $\mathcal{Q}_{d}$ denote the set of $d \times d$ ultrametric matrices of the form

$$
\left\{\left(q_{i j}\right)_{1 \leq i, j \leq d}: q_{i j}=q_{j i}, q_{i j} \geq q_{i k} \wedge q_{k j} \forall i, j, k\right\},
$$

Consider the space $\operatorname{Pr}([0,1])$ equipped with the weak* topology, so the product space $\operatorname{Pr}([0,1]) \times \mathcal{Q}_{d}$ is compact Polish. For any $Q \in \mathcal{Q}_{d}$, let $\left\{\sigma^{i}(Q): 1 \leq i \leq d\right\}$ be points in $L^{2}[0,1], \mathcal{H}$, such that the overlap matrix of these points is $Q$.

We remark here that $u_{x}^{\zeta}\left(q_{*}, \mathcal{X}_{q_{*}}\left(\sigma^{l}\right)\right)$ is explicitly given by $\frac{\mathcal{X}_{q_{*}}\left(\sigma^{l}\right)}{2\left(1+\hat{b}_{\delta}\right)}$. Furthermore, the spin distribution function $\rho_{\zeta, \sigma^{l}}^{\mathcal{X}_{q_{*}}}\left(s^{k}\right)$ for arbitrary powers $k$ can be written as polynomials of $\mathcal{X}_{q_{*}}\left(\sigma^{l}\right)$. To understand the spin distributions in the case of 1 cavity, $d$ replicas, and $e_{k, 1}=1$ for all $1 \leq k \leq d$, we see that we have to understand the continuity properties of the following functional in $\zeta$ and overlap distribution $Q$.

$$
\mathcal{R}(\zeta, Q)=\mathbb{E}\left[\prod_{i=1}^{d} u_{x}^{\zeta}\left(q_{*}, \mathcal{X}_{q_{*}}\left(\sigma^{i}(Q)\right)\right)\right] .
$$

We have the following lemma that is similar to Lemma 3.3 in [4]. We remark here that controlling other spin distributions would involve controlling powers of the same expression, and the proof would be largely unchanged.

Lemma 4.3. $\mathcal{R}$ is well-defined. We let $\zeta_{r}$ be a collection of finite replica symmetry breaking measures approaching $\zeta^{*}$ in the weak ${ }^{*}$ topology and $Q_{r}$ be a sequence of $d \times d$ matrices approaching $Q$. Then, we have that $\lim _{r \rightarrow \infty} \mathcal{R}\left(\zeta_{r}, Q_{r}\right)=\mathcal{R}\left(\zeta^{*}, Q\right)$.

Proof. In the following discussion, we will use the notation in Lemma 3.3 in [4]. Since the law of the diffusion of the local field process $\mathcal{X}$ only depends on the points $\sigma^{i}(Q)$ through the overlap distribution $Q$, the functional $\mathcal{R}\left(\zeta^{*}, Q\right)$ is well-defined.

In order to show the continuity, it would suffice to show that the diffusion corresponding to the local field process $\mathcal{X}^{\zeta_{r}, Q_{r}}$ will approach the diffusion corresponding to the limit local field process $\mathcal{X}^{\zeta^{*}, Q}$. If we let $a_{i j}^{r}(t)=\mathbb{1}\left(t \leq q_{i j}^{r}\right), b_{i j}^{r}(t,)=.\xi^{\prime \prime} \zeta_{r} u_{x}^{\zeta_{r}}(t,$.$) be the$ coefficients corresponding to the diffusion $\mathcal{X} \zeta_{r}, Q_{r}$, ideally, one would like to show that we have uniformly that $a_{i j}^{r} \rightarrow a_{i j}$ and $b_{i j}^{r}(t,.) \rightarrow b_{i j}(t,$.$) .$

However, we cannot show this uniform approach if the diffusions $b_{i j}^{r}(t,$.$) are un-$ bounded functions, which they are here. To rigorously establish our limit procedure, we define a cutoff dynamics $\tilde{b}_{i j}^{r}(t,):.=b_{i j}^{r}(t,.) \mathbb{1}[x \leq D]$ to restrict $b_{i j}$ to be a finite function. If we can show that the effect of changing $b_{i j}^{r} \rightarrow \tilde{b}_{i j}^{r}$ goes to 0 as $D$ goes to $\infty$ and that this error is uniform in $\zeta_{i}$, then using the rigorous limit ' $\tilde{b}_{i j}^{r} \rightarrow \tilde{b}_{i j}$ ' will justify ' $b_{i j}^{r} \rightarrow b_{i j}$ '.

In addition, we would need to apply finite cutoffs to the function $u_{x}$ appearing in $\mathcal{R}$ and show the uniformity of the approximation in $\zeta_{r}$. We first define

$$
u_{x}^{\zeta_{r}, B}(t, x)=u_{x}^{\zeta_{r}}(t, x) \mathbb{1}\left(\left|u_{x}^{\zeta_{r}}(t, x)\right| \leq B\right)+B \mathbb{1}\left(u_{x}^{\zeta_{r}}(t, x) \geq B\right)-B \mathbb{1}\left(u_{x}^{\zeta_{r}} \leq-B\right) .
$$

We wish to show that that

$$
\lim _{B \rightarrow \infty}\left|\mathbb{E}\left[\prod_{i=1}^{d} u_{x}^{\zeta_{r}}\left(q_{*}, \mathcal{X}_{q_{*}}^{\zeta_{r}}\left(\sigma^{i}\left(Q_{r}\right)\right)\right)\right]-\mathbb{E}\left[\prod_{i=1}^{d} u_{x}^{\zeta_{r}, B}\left(q_{*}, \mathcal{X}_{q_{*}}^{\zeta_{r}}\left(\sigma^{i}\left(Q_{r}\right)\right)\right)\right]\right| \rightarrow 0,
$$


uniformly in $\zeta_{r}$. This is a consequence of the moment bounds established in Lemma A.3 and recalling that the process $\mathcal{X}_{q_{*}}^{\zeta_{r}}$ has the same distribution as $\mathfrak{Z}^{\prime}\left(\zeta_{r}\right)$. An appropriate difference sequence along with Holder's inequality shows,

$$
\begin{aligned}
& \left|\mathbb{E}\left[\prod_{i=1}^{d} u_{x}^{\zeta_{r}}\left(q_{*}, \mathcal{X}_{q_{*}}^{\zeta_{r}}\left(\sigma^{i}\left(Q_{r}\right)\right)\right)\right]-\mathbb{E}\left[\prod_{i=1}^{d} u_{x}^{\zeta_{r}, B}\left(q_{*}, \mathcal{X}_{q_{*}}^{\zeta_{r}}\left(\sigma^{i}\left(Q_{r}\right)\right)\right)\right]\right| \leq \\
& \sum_{j=1}^{d} \mathbb{E}\left[\prod_{i=1}^{j-1} u_{x}^{\zeta_{r}}\left(q_{*}, \mathcal{X}_{q_{*}}^{\zeta_{r}}\left(\sigma^{i}\left(Q_{r}\right)\right)\right)\left(u_{x}^{\zeta_{r}}\left(q_{*}, \mathcal{X}_{q_{*}}^{\zeta_{r}}\left(\sigma^{j}\left(Q_{r}\right)\right)\right)-u_{x}^{\zeta_{r}, B}\left(q_{*}, \mathcal{X}_{q_{*}}^{\zeta_{r}}\left(\sigma^{j}\left(Q_{r}\right)\right)\right)\right)\right. \\
& \left.\prod_{i=j+1}^{d} u_{x}^{\zeta_{r}, B}\left(q_{*}, \mathcal{X}_{q_{*}}^{\zeta_{r}}\left(\sigma^{i}\left(Q_{r}\right)\right)\right)\right] \\
& \leq d\left(\mathbb{E}\left[\left|u_{x}^{\zeta_{r}}\left(q_{*}, \mathcal{X}_{q_{*}}^{\zeta_{r}}(\sigma)\right)\right|^{d}\right]\right)^{(d-1) / d}\left(\mathbb{E}\left[\left|u_{x}^{\zeta_{r}}\left(q_{*}, \mathcal{X}_{q_{*}}^{\zeta_{r}}(\sigma)\right)-u_{x}^{\zeta_{r}, B}\left(q_{*}, \mathcal{X}_{q_{*}}^{\zeta_{r}}(\sigma)\right)\right|^{d}\right]\right)^{1 / d}
\end{aligned}
$$

Recalling that $\mathcal{X}$ is distributed according to $\mathfrak{Z}^{\prime}$, we may apply the moment bounds from Lemma A.3 to show that this quantity goes to 0 as $B$ goes to $\infty$ uniformly in $\zeta_{i}$.

Using the fact that $\left|\mathcal{X}_{t}^{\zeta}\right|$ is a submartingale in $t$ (This was established for Lemma A.2 in the Appendix for $\mathfrak{Z}^{\prime}$; the proof for $\mathcal{X}$ would be similar), we may apply Doob's maximal inequality to show $\mathbb{P}\left[\sup _{0 \leq t \leq q_{*}}\left|\mathcal{X}_{t}^{\zeta_{r}}\right| \geq D\right] \leq D^{-1} \mathbb{E}\left[\left|\mathcal{X}_{t}^{\zeta_{r}}\right|\right]$. We have bounded $\mathbb{E}\left[\left|\mathcal{X}_{q_{*}}^{\zeta_{r}}\right|\right]$ through the moment bounds in Lemma A.3, again using the equivalence of the distribution of $\mathcal{X}_{q_{*}}$ with $\mathfrak{Z}^{\prime}$. If we then apply a union bound, we may then show that

$$
\lim _{D \rightarrow \infty} \mathbb{P}\left(\exists(t, i):\left|\mathcal{X}_{t}^{\zeta_{r}}\left(\sigma^{i}\left(Q_{r}\right)\right)\right| \geq D\right)=0,
$$

and this limit is uniform in $\zeta_{i}$.

Combining these two facts will allow us to see that if we instead consider the modified local field process $\tilde{\mathcal{X}}^{\zeta_{r}, Q_{r}}$ with diffusion $\tilde{a}_{i j}^{r}:=a_{i j}^{r}$ and drift $\tilde{b}_{i j}^{r}(t,):.=b_{i j}^{r}(t,.) \mathbb{1}(|x| \leq D)$, then we have that, uniformly in the $\zeta_{r}$,

$$
\lim _{B \rightarrow \infty} \lim _{D \rightarrow \infty} \mathbb{E}\left[\prod_{i=1}^{d} u_{x}^{\zeta_{r}, B}\left(q_{*}, \tilde{\mathcal{X}}_{q_{*}}^{\zeta_{r}}\left(\sigma^{i}\left(Q_{r}\right)\right)\right)\right] \rightarrow \mathbb{E}\left[\prod_{i=1}^{d} u_{x}^{\zeta_{r}}\left(q_{*}, \mathcal{X}_{q_{*}}^{\zeta_{r}}\left(\sigma^{i}\left(Q_{r}\right)\right)\right)\right] .
$$

The benefit of this representation is that we have uniform convergence for the modified diffusion coefficients $\tilde{b}$ and $\tilde{a}$ by Lemma A.4. Therefore, we can apply the Stroock-Varadhan, Theorem 11.1.4 in [16] to get the convergence

$$
\lim _{r \rightarrow \infty} \mathbb{E}\left[\prod_{i=1}^{d} u_{x}^{\zeta_{r}, B}\left(q_{*}, \tilde{\mathcal{X}}_{q_{*}}^{\zeta_{r}}\left(\sigma^{i}\left(Q_{r}\right)\right)\right)\right]=\mathbb{E}\left[\prod_{i=1}^{d} u_{x}^{\zeta^{*}, B}\left(q_{*}, \tilde{\mathcal{X}}_{q_{*}^{*}}^{\zeta^{*}}\left(\sigma^{i}(Q)\right)\right)\right] .
$$

Since we have established earlier that the limits in $B$ and $D$ can all be taken uniformly in $\zeta_{i}$, we can exchange the $r \rightarrow \infty$ limit with the $\lim _{D \rightarrow \infty}$ and $\lim _{B \rightarrow \infty}$ limits to finally derive the result $\lim _{r \rightarrow \infty} \mathcal{R}\left(\zeta_{r}, Q_{r}\right)=\mathcal{R}\left(\zeta^{*}, Q\right)$.

Now, we finish the proof of our main result.

Proof of Theorem 4.1. The goal of section 3 was to show that the moments of the spin distributions can be computed in the following manner.

$$
\lim _{N \rightarrow \infty} \mathbb{E}\left[\prod_{l=1}^{k}\left\langle\prod_{i=1}^{n}\left(\sigma_{i}^{l}\right)^{e_{l, i}}\right\rangle_{H_{N}}\right]=\lim _{m \rightarrow \infty} \mathbb{E} \prod_{l=1}^{k}\left[\sum_{\alpha_{l}} w_{\alpha_{l}} \prod_{i=1}^{n} \rho_{\zeta_{m}, h_{\alpha_{l}}}^{\left(\mathfrak{Z}_{i}\right)^{\prime}}\left(s^{e_{l, i}}\right)\right],
$$

where $\zeta_{m}$ is a series of measures supported on $\left[0, q_{*}\right]$ satisfying finite replica symmetry breaking and approaching $\zeta^{*}$ in the limit. 
The previous lemma established the fact that

$$
\lim _{m \rightarrow \infty} \mathbb{E}\left[\prod_{l=1}^{k} \prod_{i=1}^{n} \rho_{\zeta_{m}, \sigma^{l}}^{\mathcal{X}_{q_{*}}^{i}}\left(s^{e_{l, i}}\right)\right]=\mathbb{E}\left[\prod_{l=1}^{k} \prod_{i=1}^{n} \rho_{\zeta^{*}, \sigma^{l}}^{\mathcal{X}_{q_{*}}^{i}}\left(s^{e_{l, i}}\right)\right] .
$$

Lemma 4.2 showed that for the measures $\zeta_{m}$ satisfying finite replica symmetry breaking, we have

$$
\mathbb{E}\left[\prod_{l=1}^{k} \prod_{i=1}^{n} \rho_{\zeta_{m}, \sigma^{l}}^{\mathcal{X}_{q_{*}}^{i}}\left(s^{e_{l, i}}\right)\right]=\mathbb{E} \prod_{l=1}^{k}\left[\sum_{\alpha_{l}} w_{\alpha_{l}} \prod_{i=1}^{n} \rho_{\zeta_{m}, h_{\alpha_{l}}}^{\left(\mathfrak{Z}_{i}\right)^{\prime}}\left(s^{e_{l, i}}\right)\right] .
$$

Combining the last three equalities gives us the desired Theorem.

\section{A Computations for finite replica symmetry breaking}

\section{A.1 Construction of the tilted random variable}

This section will contain various estimates that we use in order to understand the random variable $\left(\mathfrak{Z}^{\prime}\right)$ and local field $\mathcal{X}$ for measures $\zeta$ that satisfy finite replica symmetry breaking.

One can apply a modified version of the Bolthausen-Sznitman invariance principle for RPCs on the random variable pair $\left(\frac{w_{\alpha} \int_{-\infty}^{\infty} e^{-\frac{1}{2}\left[1+\hat{b}_{\zeta}\right] s^{2}+\mathfrak{3}_{1}\left(h_{\alpha}\right) s} \mathrm{~d} s}{\sum_{\alpha} w_{\alpha} \int_{-\infty}^{\infty} e^{-\frac{1}{2}\left[1+\hat{b}_{\zeta}\right] s^{2}+3_{1}\left(h_{\alpha}\right) s} \mathrm{~d} s}, \mathfrak{Z}_{1}\left(h_{\alpha}\right)\right)$.

This is through a process detailed on [12][Section 4.4, Pg 131] and based off of Theorem 2.6 of [12]. Namely, let $u_{\alpha}$ be a Poisson-Dirichlet Process of parameter $m$. Let $\left(X_{\alpha}, Y_{\alpha}\right)$ are a series of $i . i . d$. variables independent of the $\left\{u_{\alpha}\right\}$ 's. Let $Y_{\alpha}^{\prime}$ be a series of i.i.d. random variables independent of the $u_{\alpha}$ with distribution $v_{m}$. The law of $v_{m}$ is given by

$$
v_{m}(B)=\frac{\mathbb{E}\left[X^{m} I(Y \in B)\right]}{\mathbb{E}\left[X^{m}\right]},
$$

where $I$ is the indicator function of the event inside the parenthesis. Then, we have the following distributional equivalence,

$$
\left(u_{\alpha} X_{\alpha}, Y_{\alpha}\right) \stackrel{d}{=}\left(\left(\mathbb{E}\left[X^{m}\right]\right)^{\frac{1}{m}} u_{\alpha}, Y_{\alpha}^{\prime}\right) .
$$

Let us now apply this re-weighting procedure to derive the random variables $\mathfrak{Z}^{\prime}$. These random variables first appeared in the proof of Lemma 3.6. Recall we wanted to understand the expression

$$
\mathbb{E}\left[\frac{\sum_{\alpha} w_{\alpha} \mathfrak{Z}\left(h_{\alpha}\right) e^{\frac{3^{2}\left(h_{\alpha}\right)}{2\left[1+\hat{b}_{\zeta}\right]}}}{\sum_{\alpha} w_{\alpha} e^{\frac{3^{2}\left(h_{\alpha}\right)}{2\left[1+\hat{b}_{\zeta}\right]}}}\right] .
$$

Recall that the variables $\mathfrak{Z}\left(h_{\alpha}\right)$ have a branching structure with $\mathfrak{Z}\left(h_{\alpha}\right)=\sum_{k=1}^{r} \mathfrak{z} \alpha^{k}$ where $\alpha^{k}$ is the ancestor of $\alpha$ at depth $k$. Each $\mathfrak{z}_{\alpha^{k}}$ is an independent Gaussian random variable with variance $\xi^{\prime}\left(q_{k}\right)-\xi^{\prime}\left(q_{k-1}\right)$.

We would now understand (A.1) by applying the previously mentioned modified Bolthausen-Sznitman invariance principle to the RPC variables $\left(w_{\alpha} e^{\frac{\mathfrak{J}^{2}\left(h_{\alpha}\right)}{2\left[1+b_{\zeta}\right]}}, \mathfrak{Z}\left(h_{\alpha}\right)\right)$.

We would be able to show that there exists a variable $\mathfrak{Z}^{\prime}\left(h_{\alpha}\right)$ with the following equality in distribution $\left(w_{\alpha} e^{\frac{\mathfrak{J}^{2}\left(h_{\alpha}\right)}{2\left[+\hat{b}_{\zeta}\right]}}, \mathfrak{Z}\left(h_{\alpha}\right)\right) \stackrel{d}{=}\left(w_{\alpha}, \mathfrak{Z}^{\prime}\left(h_{\alpha}\right)\right)$.

To define $\mathfrak{Z}^{\prime}\left(h_{\alpha}\right)$ we would require the following functions. The discussion and notation borrows heavily from Panchenko's book [12][Section 4.4]. 
We let

$$
X_{r}\left(x_{1}, \ldots, x_{r}\right)=\frac{\left(\sum_{i=1}^{r} x_{i}\right)^{2}}{2\left[1+\hat{b}_{\zeta}\right]}
$$

and define the iteration

$$
X_{p}\left(x_{1}, \ldots, x_{p}\right)=\frac{1}{\zeta\left(\left[0, q_{p}\right]\right)} \log \mathbb{E}_{p+1}\left[e^{\zeta\left(\left[0, q_{p}\right]\right) X_{p+1}\left(x_{1}, \ldots, x_{p}, x_{p+1}\right)}\right], \forall 1 \leq p \leq r-1,
$$

where $\mathbb{E}_{p+1}$ is integration with respect to the randomness of $x_{p+1}$ which is a mean zero Gaussian random variable with variance $\xi^{\prime}\left(q_{p+1}\right)-\xi^{\prime}\left(q_{p}\right)$. In the notation of [12][Ch 4], this can alternatively be seen as integration with respect to the transition kernel $G_{p+1}\left(x_{p+1}\right)=e^{-\frac{x_{p+1}^{2}}{2\left(\xi^{\prime}\left(q_{p+1}\right)-\xi^{\prime}\left(q_{p}\right)\right)}}$.

We define the modified transition kernel

$$
G_{p+1}^{\prime}\left(x^{\prime}, x_{p+1}^{\prime}\right):=G_{p+1}\left(x_{p+1}^{\prime}\right) e^{\zeta\left(\left[0, q_{p}\right]\right)\left(X_{p+1}\left(x^{\prime}, x_{p+1}^{\prime}\right)-X_{p}\left(x^{\prime}\right)\right)}
$$

With this in hand, we are now able to describe the distribution of the new variables $\mathfrak{Z}^{\prime}\left(h_{\alpha}\right): \mathfrak{Z}^{\prime}\left(h_{\alpha}\right)$ will decompose as a sum $\sum_{k=1}^{r} \mathfrak{z}_{\alpha^{k}}^{\prime}$, where we generate the terms $\mathfrak{z}_{\alpha^{k}}^{\prime}$ via an iterative process.

Consider some node $\alpha$ of the RPC and let $\alpha^{1}, \ldots, \alpha^{r}=\alpha$ be the path from the root to $\alpha$. We generate $\mathfrak{z}_{\alpha^{k}}^{\prime}$ using the values of the $\mathfrak{z}_{\alpha^{l}}^{\prime}, l<k$ and kernel $G_{k}^{\prime}$ as in the following equation.

$$
\mathbb{P}\left(\mathfrak{z}_{\alpha^{k}}^{\prime} \in A\right)=\int_{A} G_{k}^{\prime}\left(\mathfrak{z}_{\alpha^{1}}^{\prime}, \ldots, \mathfrak{z}_{\alpha^{k-1}}^{\prime}, \mathfrak{z}_{\alpha^{k}}^{\prime}\right) \mathrm{d} \mathfrak{z}_{\alpha^{k}}^{\prime}
$$

Using the equality in distribution $\left(w_{\alpha} e^{\frac{\mathfrak{Z}^{2}\left(h_{\alpha}\right)}{2\left[1+b_{\zeta} \zeta\right.}}, \mathfrak{Z}\left(h_{\alpha}\right)\right) \stackrel{d}{=}\left(w_{\alpha}, \mathfrak{Z}^{\prime}\left(h_{\alpha}\right)\right)$, we can derive that

$$
\mathbb{E}\left[\frac{\sum_{\alpha} w_{\alpha} \mathfrak{Z}\left(h_{\alpha}\right) e^{\left.\frac{\left(\mathfrak{3}\left(h_{\alpha}\right)\right)^{2}}{2[1+b} \zeta\right]}}{\sum_{\alpha} w_{\alpha} e^{\frac{\left.\left(3-h_{\alpha}\right)\right)^{2}}{2\left[1+b_{\zeta}\right]}}}\right]=\mathbb{E}\left[\frac{\sum_{\alpha} w_{\alpha} \mathfrak{Z}^{\prime}\left(h_{\alpha}\right)}{\sum_{\alpha} w_{\alpha}}\right]=\mathbb{E}\left[\mathfrak{Z}^{\prime}\left(h_{\alpha}\right)\right]
$$

In this appendix, we compute various properties of the random variable $\mathfrak{Z}^{\prime}\left(h_{\alpha}\right)$. In the computations that proceed, we will not be concerned regarding the various correlations of the $\mathfrak{Z}^{\prime}\left(h_{\alpha}\right)$ relating to the position $h_{\alpha}$ along the RPC and, instead, only consider the distribution of a single $\mathfrak{Z}^{\prime}$ with the same distribution as a single $\mathfrak{Z}^{\prime}\left(h_{\alpha}\right)$. $\mathfrak{Z}^{\prime}$ will decompose as a sum $\mathfrak{z}_{1}^{\prime}+\ldots+\mathfrak{z}_{r}^{\prime}$ where the $\mathfrak{z}_{k}^{\prime}$ are distributed according to the Kernels $G_{k}^{\prime}$. For convenience, we will define $\mathfrak{Z}^{\prime, k}:=\sum_{i=1}^{k} \mathfrak{z}_{i}^{\prime}$.

Our first lemma will involve an explicit computation of the functions $X_{p}$ for all $p$.

Lemma A.1.

$$
X_{p}\left(x_{1}, \ldots, x_{p}\right)=K_{p}+\frac{\left(\sum_{i=1}^{p} x_{i}\right)^{2}}{2\left[1+b_{p}\right]} .
$$

with some constants $K_{p}$ and $b_{p}$. The constant $b_{p}$ will satisfy the relationship $b_{p}=$ $b_{p+1}-\zeta\left(\left[0, q_{p}\right]\right)\left(\xi^{\prime}\left(q_{p+1}\right)-\xi^{\prime}\left(q_{p}\right)\right)$. Recall $b_{r}=\hat{b}_{\zeta}$.

Proof. We will prove this by induction with base case $p=r$ and going down.

We first assume the induction hypothesis for $X_{p+1}$. Letting $M_{p}=\sum_{i=1}^{p} x_{i}$ and 
recalling the constant $1+b_{p}=\left[1+b_{p+1}\right]-\zeta\left(\left[0, q_{p}\right]\right)\left(\xi^{\prime}\left(q_{p+1}\right)-\xi^{\prime}\left(q_{p}\right)\right)$, we have

$$
\begin{aligned}
& X_{p}\left(x_{1}, \ldots, x_{p}\right)-K_{p+1} \\
& =\frac{1}{\zeta\left(\left[0, q_{p}\right]\right)} \log \frac{\sqrt{\xi^{\prime}\left(q_{p+1}\right)-\xi^{\prime}\left(q_{p}\right)}}{\sqrt{2 \pi}} \int e^{\frac{\zeta\left(\left[0, q_{p}\right]\right)}{2\left[1+b_{p+1}\right]}\left(M_{p}^{2}+2 x_{p+1} M_{p}+x_{p+1}^{2}\right)} e^{-\frac{x_{p+1}^{2}}{2\left(\xi^{\prime}\left(q_{p+1}\right)-\xi^{\prime}\left(q_{p}\right)\right)}} \mathrm{d} x_{p+1} \\
& =\frac{1}{\zeta\left[0, q_{p}\right]} \log \frac{\sqrt{\xi^{\prime}\left(q_{p+1}\right)-\xi^{\prime}\left(q_{p}\right)}}{\sqrt{2 \pi}} \int \exp \left\{M_{p}^{2}\left[\frac{\zeta\left(\left[0, q_{p}\right]\right)^{2}\left(\xi^{\prime}\left(q_{p+1}\right)-\xi^{\prime}\left(q_{p}\right)\right)}{2\left(1+b_{p+1}\right)\left(1+b_{p}\right)}+\frac{\zeta\left(\left[0, q_{p}\right]\right)}{2\left[1+b_{p+1}\right]}\right]\right. \\
& \left.\quad-\frac{1+b_{p}}{2\left[1+b_{p+1}\right]\left(\xi^{\prime}\left(q_{p+1}\right)-\xi^{\prime}\left(q_{p}\right)\right)}\left(x_{p+1}-\tilde{M}_{p}\right)^{2}\right\} \mathrm{d} x_{p+1},
\end{aligned}
$$

where

$$
\tilde{M}_{p}=M_{p} \frac{\zeta\left(\left[0, q_{p}\right]\right)\left(\xi^{\prime}\left(q_{p+1}\right)-\xi^{\prime}\left(q_{p}\right)\right)}{1+b_{p}} .
$$

For $\delta\left(\left[0, q_{p}\right]\right)=0$, the above should be interpreted as the $\lim _{\delta\left(\left[0, q_{p}\right]\right) \rightarrow 0}$ limit of the above expression. Direct calculation can show the expression has a valid limit.

We can integrate the Gaussian above provided we have $1+b_{p} \geq 0$. We can also evaluate the constant factor in front of the remaining $M_{p}^{2}$ in the exponential as

$$
\frac{\zeta\left(\left[0, q_{p}\right]\right)^{2}\left(\xi^{\prime}\left(q_{p+1}\right)-\xi^{\prime}\left(q_{p}\right)\right)}{2\left(1+b_{p+1}\right)\left(1+b_{p}\right)}+\frac{\zeta\left(\left[0, q_{p}\right]\right)}{2\left[1+b_{p+1}\right]}=\frac{\zeta\left(\left[0, q_{p}\right]\right)}{2\left(1+b_{p}\right)} .
$$

Using this we see that

$$
X_{p}\left(x_{1}, \ldots, x_{p}\right)=K_{p}+\frac{M_{p}^{2}}{2\left[1+b_{p}\right]} .
$$

Notice that $b_{p}$ is increasing in $p$ (namely, higher the $p$ the higher the value of $b_{p}$ ). The induction will be finished provided we have that $1+b_{0}$ is positive.

We can express $1+b_{0}=1+\sum_{i=0}^{r} \zeta\left(\left\{q_{p}\right\}\right)\left(1-q_{p}\right) \xi^{\prime}\left(q_{p}\right)$. This is manifestly positive.

The implication of this lemma is that we see that the modified transition kernel has the following density,

$$
G_{p+1}^{\prime}\left(x^{\prime}, x_{p+1}^{\prime}\right) \propto e^{-\frac{\left(x_{p+1}^{\prime}\right)^{2}}{2\left(\xi^{\prime}\left(q_{p+1}\right)-\xi^{\prime}\left(q_{p}\right)\right)}} e^{\zeta\left(\left[0, q_{p}\right]\right) \frac{\left(\frac{\left(\sum_{k=1}^{p+1} x_{k}^{\prime}\right)^{2}}{2\left[1+b_{p+1}\right]}\right.}{2}}
$$

Lemma A.2. The process $\left|\mathfrak{Z}^{\prime, k}\right|=\left|\sum_{j=1}^{k} \mathfrak{z}_{j}^{\prime}\right|$ is a submartingale.

Proof. We first remark that the transition Kernel $G_{k+1}^{\prime}$ will be a function that depends only on the values $\mathfrak{z}_{1}^{\prime}, \mathfrak{z}_{2}^{\prime}, \ldots, \mathfrak{z}_{k}^{\prime}$ through the sum $\mathfrak{Z}^{\prime, k}$. We now have to perform the explicit computation which will allow us to understand the value of $\mathfrak{z}_{k+1}^{\prime}$.

We first remark that trivially when $\mathfrak{Z}^{\prime, k}$ is positive, we have that

$$
\mathbb{E}\left[\left|\mathfrak{Z}^{\prime, k+1}\right||| \mathfrak{Z}^{\prime, k} \mid\right] \geq \mathbb{E}\left[\left|\mathfrak{Z}^{\prime, k}\right|+\mathfrak{z}_{k+1}^{\prime}|| \mathfrak{Z}^{\prime, k} \mid\right] .
$$

Notice that since the Kernel does not depend on the sign of $\mathfrak{Z}^{\prime, k}$, we may as well assume that $\mathfrak{Z}^{\prime, k}$ is positive when computing expectations.

We will have proved $\left|\mathfrak{Z}^{\prime, k}\right|$ is a submartingale when we establish

$$
\mathbb{E}\left[\mathfrak{Z}^{\prime, k}+\mathfrak{z}_{k+1}^{\prime} \mid \mathfrak{Z}^{\prime, k}\right]>\mathfrak{Z}^{\prime, k},
$$

when $\mathfrak{Z}^{\prime, k}$ is positive. 
Let us now compute the necessary integral

$$
\begin{aligned}
\mathbb{E}\left[\mathfrak{z}_{k+1}^{\prime} \mid \mathfrak{Z}^{\prime, k}\right] & =\frac{\int z e^{\zeta\left(\left[0, q_{k}\right]\right) \frac{\left(\mathfrak{J}^{\prime}, k+z\right)^{2}}{2\left[1+b_{k+1}\right]}} e^{-\frac{z^{2}}{2\left(\xi^{\prime}\left(q_{k+1}\right)-\xi^{\prime}\left(q_{k}\right)\right)}} \mathrm{d} z}{\int e^{\zeta\left(\left[0, q_{k}\right]\right) \frac{\left(\mathfrak{3}^{\prime}, k+z\right)^{2}}{2\left[1+b_{k+1}\right]}} e^{-\frac{z^{2}}{2\left(\xi^{\prime}\left(q_{k+1}\right)-\xi^{\prime}\left(q_{k}\right)\right)}} \mathrm{d} z} \\
& =\mathfrak{Z}^{\prime, k} \zeta\left(\left[0, q_{k}\right]\right) \frac{\xi^{\prime}\left(q_{k+1}\right)-\xi^{\prime}\left(q_{k}\right)}{1+b_{k}} .
\end{aligned}
$$

Clearly, this will be positive as we have assumed $\mathfrak{Z}^{\prime, k}$ to be positive and we have proved that the sequence $\mathfrak{Z}^{\prime, k}$ is a submartingale.

In the following lemmas, we want to study the behavior of the random variable $\mathfrak{Z}^{\prime}$ as a function of the approximating measure $\zeta$. We will use the notation $\mathfrak{Z}^{\prime}(\zeta)$ to specifically denote the random variable $\mathfrak{Z}^{\prime}$ when it is derived using the base measure $\zeta$.

Lemma A.3. There exists a constant $B_{p}$ that depends only on $\xi$ and $p$ such that for all $\zeta$ such that $\sup \operatorname{supp} \zeta=q_{*}$, we have $\mathbb{E}\left[\left(\mathfrak{Z}^{\prime}(\zeta)\right)^{p}\right] \leq B_{p}$.

Proof. This will be the consequence of computing moment bounds, which is an exercise in computing the expectation level by level. We will demonstrate in detail the computation for the second moment; we can bound higher moments by the same method. We will prove a recursive relation for $\mathbb{E}\left[\left(\mathfrak{Z}^{\prime, k+1}\right)^{2}\right]$ in terms of $\mathbb{E}\left[\left(\mathfrak{Z}^{\prime, k}\right)^{2}\right]$ by direct integration.

$$
\begin{aligned}
& \mathbb{E}\left[\mathfrak{Z}^{\prime, k+1}(\zeta)^{2}\right]=\frac{\left(1+b_{k+1}\right)^{2}}{\left(1+b_{k}\right)} \mathbb{E}\left[\left(\mathfrak{Z}^{\prime, k}(\zeta)\right)^{2}\right] \\
& \quad+2 \frac{1+b_{k+1}}{1+b_{k}} \mathbb{E}\left[\mathfrak{Z}^{\prime, k}(\zeta) \mathbb{E}\left[\left(\mathfrak{z}_{k+1}^{\prime}(\zeta)-\zeta\left(\left[0, q_{k}\right]\right) \frac{\xi^{\prime}\left(q_{k+1}\right)-\xi^{\prime}\left(q_{k}\right)}{1+b_{k}} \mathfrak{Z}^{\prime, k}(\zeta)\right) \mid \mathfrak{Z}^{\prime, k}(\zeta)\right]\right] \\
& \quad+\mathbb{E}\left[\mathbb{E}\left[\left(\mathfrak{z}_{k+1}^{\prime}(\zeta)-\zeta\left(\left[0, q_{k}\right]\right) \frac{\xi^{\prime}\left(q_{k+1}\right)-\xi^{\prime}\left(q_{k}\right)}{1+b_{k}} \mathfrak{Z}^{\prime, k}(\zeta)\right)^{2} \mid \mathfrak{Z}^{\prime, k}(\zeta)\right]\right] .
\end{aligned}
$$

From examining the transition kernel (A.10), we observe that $\mathfrak{z}_{k+1}^{\prime}$ is a Gaussian with mean tilted to $\zeta\left(\left[0, q_{k}\right]\right) \frac{\xi^{\prime}\left(q_{k+1}\right)-\xi^{\prime}\left(q_{k}\right)}{1+b_{k}} \mathfrak{Z}^{\prime, k}(\zeta)$. This explains the expansion given in the equation above.

Under this rescaling, we note that the computation of the first moment in the second line of (A.12) is 0 . The third line will give us the variance of the Gaussian $\mathfrak{z}_{k+1}^{\prime}$. This variance is given by

$$
\frac{\left(1+b_{k+1}\right)\left(\xi^{\prime}\left(q_{k+1}\right)-\xi^{\prime}\left(q_{k}\right)\right)}{\left(1+b_{k}\right)}
$$

In summary, we thus, have the inductive relation

$$
\mathbb{E}\left[\left(\mathfrak{Z}^{\prime, k+1}(\zeta)\right)^{2}\right]=\left(\frac{1+b_{k+1}}{1+b_{k}}\right)^{2} \mathbb{E}\left[\mathfrak{Z}^{\prime, k}(\zeta)^{2}\right]+\frac{\left(1+b_{k+1}\right)\left(\xi^{\prime}\left(q_{k+1}\right)-\xi^{\prime}\left(q_{k}\right)\right)}{\left(1+b_{k}\right)} .
$$

We see that the prefactor $\frac{\left(1+b_{k+1}\right)^{2}}{\left(1+b_{k}\right)^{2}}$ will telescope upon recursion.

This gives us the relation,

$$
\mathbb{E}\left[\left(\mathfrak{Z}^{\prime, r}(\zeta)\right)^{2}\right]=\sum_{k=1}^{r} \frac{\left(1+b_{r}\right)^{2}}{\left(1+b_{k}\right)^{2}} \frac{\left(1+b_{k}\right)}{\left(1+b_{k-1}\right)}\left(\xi^{\prime}\left(q_{k}\right)-\xi^{\prime}\left(q_{k-1}\right)\right) .
$$

We observe that since $1+b_{k}>1$ for all $k$ and $1+b_{k}<1+b_{r}<1+\xi^{\prime}\left(q_{*}\right)$, we may bound all prefactors involving $b$ that appear by a constant only depending on $\xi$. As a result, we 
see that

$$
\mathbb{E}\left[\left(\mathfrak{Z}^{\prime, r}\right)^{2}\right] \leq\left(1+\xi^{\prime}\left(q_{*}\right)\right)^{3} \sum_{k=1}^{r}\left(\xi^{\prime}\left(q_{k}\right)-\xi^{\prime}\left(q_{k-1}\right) \leq\left(1+\xi^{\prime}\left(q_{*}\right)\right)^{3} \xi^{\prime}\left(q_{*}\right) .\right.
$$

This bound only depends on the covariance structure.

For higher moments, we also have a recursive relation,

$$
\begin{aligned}
& \mathbb{E}\left[\left(\mathfrak{Z}^{\prime, k+1}\right)^{p}\right]= \\
& \sum_{j=0}^{p}\left(\frac{1+b_{k+1}}{1+b_{k}}\right)^{j} \mathbb{E}\left[\left(\mathfrak{Z}^{\prime, k}\right)^{j} \mathbb{E}\left[\left(\mathfrak{z}_{k+1}^{\prime}-\zeta\left(\left[0, q_{k}\right] \frac{\xi^{\prime}\left(q_{k+1}\right)-\xi^{\prime}\left(q_{k}\right)}{1+b_{k}} \mathfrak{Z}^{\prime, k}(\zeta)\right)^{p-j} \mid \mathfrak{Z}^{\prime, k}(\zeta)\right]\right] .\right.
\end{aligned}
$$

We may evaluate

$$
\begin{aligned}
& \mathbb{E}\left[\left(\mathfrak{z}_{k+1}^{\prime}-\zeta\left(\left[0, q_{k}\right]\right) \frac{\xi^{\prime}\left(q_{k+1}\right)-\xi^{\prime}\left(q_{k}\right)}{1+b_{k}} \mathfrak{Z}^{\prime, k}(\zeta)\right)^{p-j} \mid \mathfrak{Z}^{\prime, k}\right] \\
& =\mathcal{P}_{p-j}\left(\frac{\left(1+b_{k+1}\right)\left(\xi^{\prime}\left(q_{k+1}\right)-\xi^{\prime}\left(q_{k}\right)\right)}{2\left(1+b_{k}\right)}\right)^{p-j},
\end{aligned}
$$

where $\mathcal{P}_{p-j}$ is the $p-j$ th moment of the standard normal random variable.

Again, we may recursively apply (A.16) and see that the coefficients $\left(\frac{1+b_{k+1}}{1+b_{k}}\right)^{p}$ is telescoping. After applying the bound $\frac{1+b_{j}}{1+b_{k}} \leq\left(1+\xi^{\prime}\left(q_{*}\right)\right), j>k$ to all such ratios that appear, we see that we have,

$$
\mathbb{E}\left[\left(\mathfrak{Z}^{\prime, r}(\zeta)\right)^{p}\right] \leq C \sum_{j=0}^{p-1} \sum_{l=0}^{r-1} \mathbb{E}\left[\left(\mathfrak{Z}^{\prime, l}(\zeta)\right)^{j}\right] \mathcal{P}_{p-j}\left(\xi^{\prime}\left(q_{l+1}\right)-\xi^{\prime}\left(q_{l}\right)\right)^{p-j} .
$$

By inducting on the power $p$, one can bound $\mathbb{E}\left[\left(\mathfrak{Z}^{\prime}, l\right)^{j}\right]$ by some constant that depends on $\xi$ and is independent of $\zeta$.

We may also bound

$$
\sum_{l=0}^{r-1}\left(\xi^{\prime}\left(q_{l+1}\right)-\xi^{\prime}\left(q_{l}\right)\right)^{p-j} \leq\left(\sum_{l=0}^{r-1} \xi^{\prime}\left(q_{l+1}\right)-\xi^{\prime}\left(q_{l}\right)\right)^{p-j} \leq \xi^{\prime}\left(q_{*}\right)^{p-j},
$$

where we used the positivity of the differences $\xi^{\prime}\left(q_{l+1}\right)-\xi^{\prime}\left(q_{l}\right)$.

Ultimately, this bound $\mathbb{E}\left[\left(\mathfrak{Z}^{\prime, r}(\zeta)\right)^{p}\right]$ by a quantity that depends on $\xi$ but not on $\zeta$.

\section{A.2 The Parisi PDE}

The properties of the random variables $Z^{\prime}$ that we have defined are closely related to the solutions of an associated Parisi PDE at approximating measure $\zeta$ with support at points $0=q_{0} \leq q_{1} \leq \ldots \leq q_{r}=q_{*} \leq 1$. We use the notation $q_{r+1}=1$ where appropriate.

$$
\partial_{t} u^{\zeta}(t, x)=-\frac{\xi^{\prime \prime}(t)}{2}\left(\partial_{x}^{2} u^{\zeta}(t, x)+\zeta([0, x])\left(\partial_{x} u^{\zeta}(t, x)\right)^{2}\right) .
$$

with initial data given by

$$
u^{\zeta}(1, x)=\frac{x^{2}}{2\left[1+b_{\zeta}\right]} .
$$

with $b_{\zeta}=\sum_{i=1}^{r+1}\left[q_{i} \xi^{\prime}\left(q_{i}\right)-q_{i-1} \xi^{\prime}\left(q_{i-1}\right)\right] \zeta\left(\left[0, q_{i-1}\right]\right)$. 
At $\zeta$ satisfying finite replica symmetry breaking, as we have assumed here, we have the Cole-Hopf solution of the Parisi PDE. Namely, for $t$ in between $q_{j} \leq t<q_{j+1}$, we have

$$
u^{\zeta}(t, x)=\frac{1}{\zeta\left(\left[0, q_{j}\right]\right)} \log \mathbb{E}\left[\exp \zeta\left(\left[0, q_{j}\right]\right) u^{\zeta}\left(q_{j+1}, x+z \sqrt{\left(\xi^{\prime}\left(q_{j+1}\right)-\xi^{\prime}(t)\right)}\right)\right] .
$$

where $z$ is a standard Gaussian random variable. The computation of the integral is very similar to what was performed in the computation of the function $X_{j}\left(x_{1}, \ldots, x_{j}\right)$ aside from the constant factor shift.

We see that for the specific values $t=q_{j}$, we have

$$
u^{\zeta}\left(q_{j}, x\right)=\frac{x^{2}}{2\left(1+b_{j}\right)}+\sum_{k=j}^{r} \frac{1}{2 \zeta\left(\left[0, q_{k}\right]\right)} \log \frac{1+b_{k+1}}{1+b_{k}},
$$

and for values $q_{j} \leq t \leq q_{j+1}$ in between, we have

$$
\begin{aligned}
u^{\zeta}(t, x) & =\frac{x^{2}}{2\left(1+b_{j+1}-\zeta\left(\left[0, q_{j}\right]\right)\left(\xi^{\prime}\left(q_{j+1}\right)-\xi^{\prime}(t)\right)\right)}+\sum_{k=j+1}^{r} \frac{1}{2 \zeta\left(\left[0, q_{k}\right]\right)} \log \frac{1+b_{k+1}}{1+b_{k}} \\
& +\frac{1}{2 \zeta\left(\left[0, q_{j}\right]\right)} \log \frac{1+b_{j+1}}{1+b_{j+1}-\zeta\left(\left[0, q_{j}\right]\right)\left(\xi^{\prime}\left(q_{j+1}\right)-\xi^{\prime}(t)\right)} .
\end{aligned}
$$

When $\zeta\left(\left[0, q_{j}\right]\right)=0$, the last quantity should be understood as $\frac{\xi^{\prime}\left(q_{j+1}\right)-\xi^{\prime}(t)}{2\left(1+b_{j+1}\right)}$.

Observe that from the formula given above, if we let $\zeta_{i}$ be a sequence of measures supported on $\left[0, q_{*}\right]$ that approach a limit $\zeta^{*}$ in the weak ${ }^{*}$ topology, then the solutions $u^{\zeta_{i}}(t, x)$ approach a limit $u^{\zeta^{*}}(t, x)$ with formula given by

$$
\begin{aligned}
u^{\zeta^{*}}(t, x) & =\frac{x^{2}}{2\left(1+\int_{0}^{1} \zeta^{*}([0, l])\left(l \xi^{\prime \prime}(l)+\xi^{\prime}(l)\right) \mathrm{d} l-\int_{t}^{1} \zeta^{*}([0, l]) \xi^{\prime \prime}(l) \mathrm{d} l\right)} \\
& +\frac{1}{2} \int_{t}^{1} \frac{\xi^{\prime \prime}(l)}{1+\int_{0}^{1} \zeta^{*}([0, n])\left(n \xi^{\prime \prime}(n)+\xi^{\prime}(n)\right) \mathrm{d} n-\int_{l}^{1} \zeta^{*}([0, n]) \xi^{\prime \prime}(n) \mathrm{d} n} \mathrm{~d} l
\end{aligned}
$$

One can check from an integration by parts formula that

$$
\begin{aligned}
& \int_{0}^{1} \zeta^{*}([0, l])\left(l \xi^{\prime}(l)\right)^{\prime} \mathrm{d} l+\int_{0}^{1}\left(\zeta^{*}([0, l])\right)^{\prime} l \xi^{\prime}(l) \mathrm{d} l=\zeta^{*}([0,1]) \xi^{\prime}(1), \\
& \int_{0}^{1} \zeta^{*}([0, l])\left(\xi^{\prime}(l)\right)^{\prime} \mathrm{d} l+\int_{0}^{1}\left(\zeta^{*}([0, l])\right)^{\prime} \xi^{\prime}(l) \mathrm{d} l=\zeta^{*}([0,1]) \xi^{\prime}(1)-\zeta^{*}([0,0]) \xi^{\prime}(0) .
\end{aligned}
$$

Thus, we have

$$
\begin{aligned}
& \int_{0}^{1} \zeta^{*}([0, l])\left(l \xi^{\prime \prime}(l)+\xi^{\prime}(l)\right) \mathrm{d} l-\int_{0}^{1} \zeta^{*}([0, l]) \xi^{\prime \prime}(l) \mathrm{d} l \\
& =\int_{0}^{1}\left(\zeta^{*}([0, l])\right)^{\prime}(1-l) \xi^{\prime}(l) \mathrm{d} l+\zeta^{*}([0,0]) \xi^{\prime}(0) .
\end{aligned}
$$

The quantity above is clearly positive and, as such, we can see that the denominator in (A.24) is always well-defined for our initial choice of constant.

We contain these observations in a lemma.

Lemma A.4. Consider a sequence of measures $\zeta_{i}$ on $([0,1]$ satisfying finite replica symmetry breaking. Suppose the set $\zeta_{i}$ has some weak limit $\zeta^{*}$ in the weak ${ }^{*}$ topology. Then on any compact region $\mathcal{C}$ of $\mathbb{R} \times[0,1]$ we have uniform convergence of the solution of the Parisi PDE (A.19) to the limit solutions.

$$
u^{\zeta_{i}}(x, t) \rightarrow u^{\zeta^{*}}(x, t),
$$

uniformly in $\mathcal{C}$. 
Spin distributions for spherical spin glasses

\section{References}

[1] L.P. Arguin, Spin Glass Computations and Ruelle's Probability Cascades, Journal of Statistical Physics, 126.4,951-976 (2007) MR2311892

[2] A. Auffinger, W. Chen, On Properties of Parisi Measures, Probability Theory and Related Fields 161(2015), No 3-4, 1429-1444 MR3334282

[3] A. Auffinger, W. Chen, The Parisi formula has a unique minimizer, Communications in Mathematical Physics, 335(3), 1429-1444. MR3320318

[4] A. Auffinger, A. Jagannath, , On Spin Distributions for Generic p-spin Models, J. Stat Phys 174(2019), No. 316 MR3910895

[5] E. Bolthausen, A Sznitman, On Ruelle's Probability Cascade and an Abstract Cavity Method, Comm. Math. Phys. MR1652734

[6] L.N. Dobvysh, V.N. Sudakov, Gram-de Finetti Matrices, Zap. Nauchn. Sem. Leningrad Otdel. Mat. Inst. Stekov 119(1982), 77-86 MR0666087

[7] M. Mezard, G. Parisi, N. Sourlas, G. Toulouse, M.A. Virasoro, On the Nature of the Spin Glass Phase, Phys. Rev Letters(1984), No. 52, 1156

[8] M. Mezard, G. Parisi, N. Sourlas, G. Toulouse, M.A. Virasoro, Replica Symmetry Breaking and the Nature of the Spin Glass Phase, J. de Physique 45, 843 MR0746889

[9] M. Mezard, G. Parisi,M.A. Virasoro, Sping Glass Theory and Beyond, World Scientific Lecture Notes in Physics, 9. World Scientific Publishing Co., Inc, Teaneck, NJ MR1026102

[10] D. Panchenko, Spin Glass Models from the Point of View of Spin Distributions, Annals of Probability 41(2013), No. 3A, 1315-1361 MR3098679

[11] D. Panchenko, The Parisi Ultrametricity Conjecture, Annals of Mathematics 177(2013), Issue 1, 383-393 MR2999044

[12] D. Panchenko, The Sherrington-Kirkpatrick Model, Springer Monographs in Mathematics, Springer New York Heidelberg, 2013 MR3052333

[13] G. Parisi, A Sequence of Approximate Solutions to the SK Model for Spin Glasses, J. Phys. A. NO. 13, L-115

[14] G. Parisi, Infinite Number of Order Parameters for Spin-Glasses, Phys. Rev Letters (1979), No. 43, 1754-1756 MR0702601

197(1998),No. 2, 247-276

[15] D. Sherrington, S. Kirkpatrick, A Solvable Model of Spin Glass, Phys. Rev Letters(1975), No. 35, 1792-1796

[16] D. Stroock, S.R.S. Varadhan, Multidimensional Diffusion Processes, volume 233(1979), Springer Science \& Business Media MR0532498

[17] E. Subag, The Geometry of the Gibbs Measure of Pure Spherical Spin Glasses, Invenciones Mathematicae 210(2017), Issue 1, 135-209 MR3698341

[18] E. Subag, O. Zeitouni, The Extremal Process of Critical Points of the Pure p-Spin Spherical Spin Glass Model, Probability Theory and Related Fields 168(2017),Issue 3-4, 773-820 MR3663631

[19] M. Talagrand, Free Energy of the Spherical Mean Field Model, Prob. Theory Relat. Fields 134 (2006), 339-382 MR2226885

[20] M. Talagrand, The Parisi Formula, Annals of Mathematics 163(2006), Issue 1, 221-263 MR2195134

Acknowledgments. The author is grateful to Aukosh Jagannath for introducing him to this problem and for useful discussions. 


\section{Electronic Journal of Probability Electronic Communications in Probability}

\section{Advantages of publishing in EJP-ECP}

- Very high standards

- Free for authors, free for readers

- Quick publication (no backlog)

- Secure publication $\left(\mathrm{LOCKSS}^{1}\right)$

- Easy interface (EJMS²)

\section{Economical model of EJP-ECP}

- Non profit, sponsored by $\mathrm{IMS}^{3}, \mathrm{BS}^{4}$, ProjectEuclid ${ }^{5}$

- Purely electronic

\section{Help keep the journal free and vigorous}

- Donate to the IMS open access fund ${ }^{6}$ (click here to donate!)

- Submit your best articles to EJP-ECP

- Choose EJP-ECP over for-profit journals

\footnotetext{
${ }^{1}$ LOCKSS: Lots of Copies Keep Stuff Safe http://www. lockss.org/

${ }^{2}$ EJMS: Electronic Journal Management System http://www.vtex.lt/en/ejms.html

${ }^{3}$ IMS: Institute of Mathematical Statistics http://www.imstat.org/

${ }^{4}$ BS: Bernoulli Society http://www. bernoulli-society.org/

${ }^{5}$ Project Euclid: https://projecteuclid.org/

${ }^{6}$ IMS Open Access Fund: http://www.imstat.org/publications/open.htm
} 